Florida International University FIU Digital Commons

7-16-2008

\title{
Freeway Travel Time Estimation and Prediction Using Dynamic Neural Networks
}

Luou Shen

Florida International University, 1shen001@fiu.edu

DOI: $10.25148 /$ etd.FI08121916

Follow this and additional works at: https://digitalcommons.fiu.edu/etd

\section{Recommended Citation}

Shen, Luou, "Freeway Travel Time Estimation and Prediction Using Dynamic Neural Networks" (2008). FIU Electronic Theses and Dissertations. 17.

https://digitalcommons.fiu.edu/etd/17

This work is brought to you for free and open access by the University Graduate School at FIU Digital Commons. It has been accepted for inclusion in FIU Electronic Theses and Dissertations by an authorized administrator of FIU Digital Commons. For more information, please contact dcc@fiu.edu. 


\section{FLORIDA INTERNATIONAL UNIVERSITY}

Miami, Florida

FREEWAY TRAVEL TIME ESTIMATION AND PREDICTION USING DYNAMIC NEURAL NETWORKS

A dissertation submitted in partial fulfillment of the requirements for the degree of DOCTOR OF PHILOSOPHY

in

CIVIL ENGINEERING

by

Luou Shen 
To: $\quad$ Interim Dean Amir Mirmiran

College of Engineering and Computing

This dissertation, written by Luou Shen, and entitled Freeway Travel Time Estimation and Prediction Using Dynamic Neural Networks, having been approved in respect to style and intellectual content, is referred to you for judgment.

We have read this dissertation and recommend that it be approved.

Fang Zhao

Albert Gan

Zhenmin Chen

Mohammed Hadi, Major Professor

Date of Defense: July 16, 2008

The dissertation of Luou Shen is approved.

\begin{tabular}{r} 
Interim Dean Amir Mirmiran \\
College of Engineering and Computing \\
\hline Dean George Walker \\
University Graduate School
\end{tabular}

Florida International University, 2008 


\section{DEDICATION}

I dedicate this dissertation to my family for their love, understanding, and constant support. 


\section{ACKNOWLEDGMENTS}

I would like to take this opportunity to express my gratitude to my advisor, Dr. Mohammed Hadi, for his guidance, encouragement, and support throughout my doctoral study, and for his many nights and weekends extended to the direction and review of my research papers and dissertation. A special thank goes to Dr. Fang Zhao, for her invaluable advice and continuous encouragement on my dissertation research.

I am also greatly indebted to the rest of my dissertation committee members, Dr. Albert Gan, and Dr. Zhenmin Chen for serving on my committee, and for their comments, suggestions, and interest in my research.

A special note of appreciation is due to Mr. Dong Chen and the District 4 ITS Office of the Florida Department of Transportation for providing detector data for this research.

Last but certainly not least, I would like to thank Florida International University for providing the financial support for my graduate study and for this dissertation research. Thank you! 
ABSTRACT OF THE DISSERTATION

FREEWAY TRAVEL TIME ESTIMATION AND PREDICTION

USING DYNAMIC NEURAL NETWORKS

by

Luou Shen

Florida International University, 2008

Miami, Florida

Professor Mohammed Hadi, Major Professor

Providing transportation system operators and travelers with accurate travel time information allows them to make more informed decisions, yielding benefits for individual travelers and for the entire transportation system. Most existing advanced traveler information systems (ATIS) and advanced traffic management systems (ATMS) use instantaneous travel time values estimated based on the current measurements, assuming that traffic conditions remain constant in the near future. For more effective applications, it has been proposed that ATIS and ATMS should use travel times predicted for short-term future conditions rather than instantaneous travel times measured or estimated for current conditions.

This dissertation research investigates short-term freeway travel time prediction using Dynamic Neural Networks (DNN) based on traffic detector data collected by radar traffic detectors installed along a freeway corridor. DNN comprises a class of neural networks that are particularly suitable for predicting variables like travel time, but has not been adequately investigated for this purpose. Before this investigation, it was necessary to identifying methods for data imputation to account for missing data usually 
encountered when collecting data using traffic detectors. It was also necessary to identify a method to estimate the travel time on the freeway corridor based on data collected using point traffic detectors. A new travel time estimation method referred to as the Piecewise Constant Acceleration Based (PCAB) method was developed and compared with other methods reported in the literatures. The results show that one of the simple travel time estimation methods (the average speed method) can work as well as the PCAB method, and both of them out-perform other methods. This study also compared the travel time prediction performance of three different DNN topologies with different memory setups. The results show that one DNN topology (the time-delay neural networks) out-performs the other two DNN topologies for the investigated prediction problem. This topology also performs slightly better than the simple multilayer perceptron (MLP) neural network topology that has been used in a number of previous studies for travel time prediction. 


\section{TABLE OF CONTENTS}

CHAPTER

PAGE

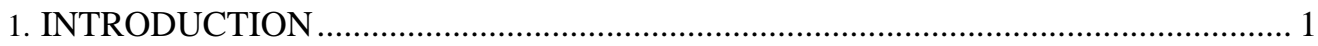

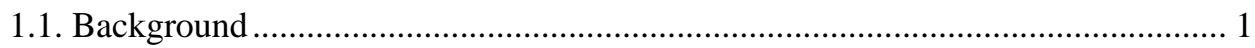

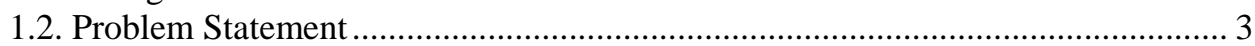

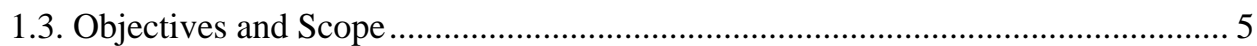

1.4. Dissertation Organization ............................................................................... 5

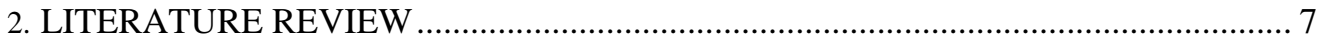

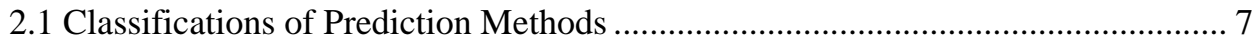

2.1.1. Scope of Application........................................................................................ 7

2.1.2. Prediction Horizon and Step ........................................................................... 7

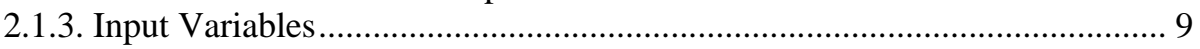

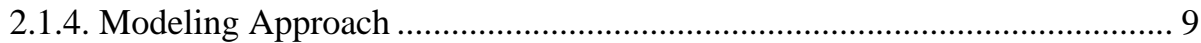

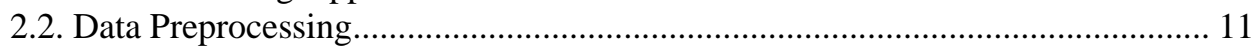

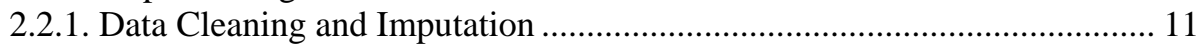

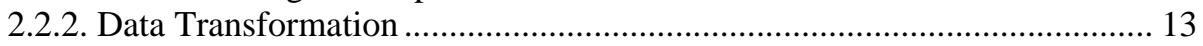

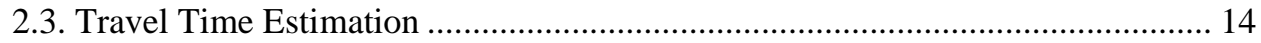

2.3.1. Trajectory Methods........................................................................................ 14

2.3.2. Traffic Flow Theory Method ....................................................................... 17

2.4. Travel Time Prediction .......................................................................................... 18

2.4.1. Methods Based on Historical Data ............................................................. 18

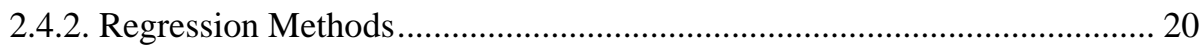

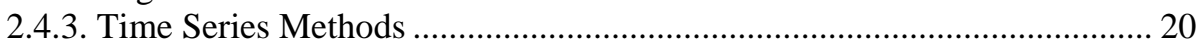

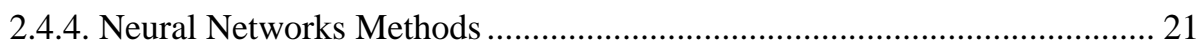

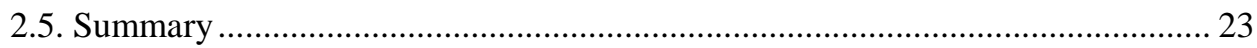

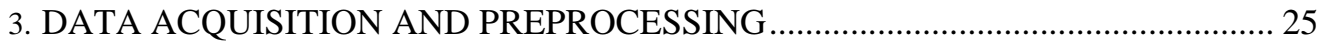

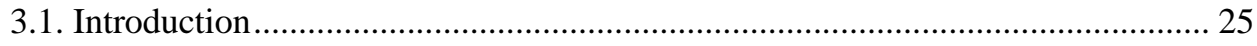

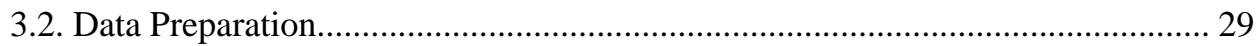

3.3. Data Imputation ………………………................................................................. 33

3.4. Speed Transformation.................................................................................. 39

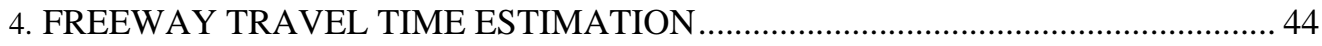

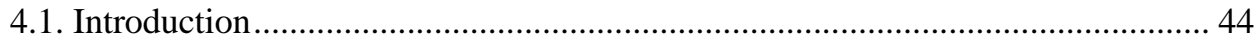

4.2. Validation of Travel Time Estimation Method with Simulation Data ................ 48

4.3. Validation of Data Preprocessing Method with Simulation Data........................ 53

4.3.1. Validation of Data Imputation Method......................................................... 53

4.3.2. Validation of Speed Transformation Equation .......................................... 56

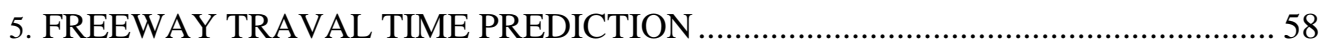

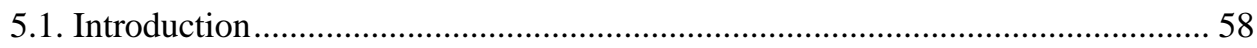

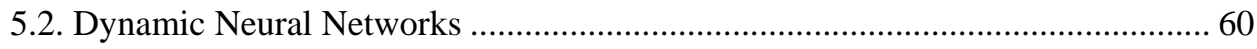

5.2.1. Short-term Memory .................................................................................... 61 
5.2.2. Feedback Application .............................................................................62

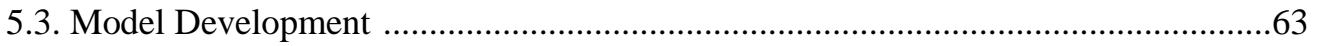

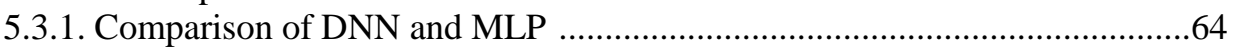

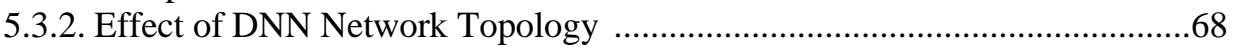

5.4. Effect of Prediction Horizon .................................................................................

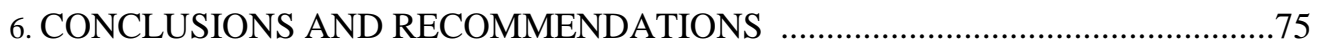

6.1. Summary of Research Results and Conclusions ............................................75

6.2. Recommendations for Future Development ...................................................77

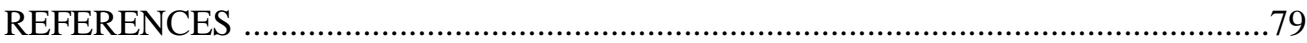

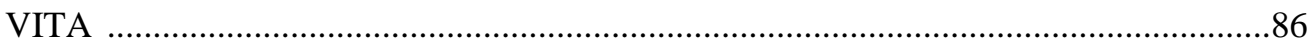




\section{LIST OF TABLES}

TABLE

Table 3.1: Example of Traffic Detector Data Records ..................................................... 26

Table 3.2: Sample Incident Data.................................................................................... 29

Table 3.3: Rearranged Traffic Detector Data Table ....................................................... 30

Table 3.4: Missing Percent of Training Data.................................................................... 32

Table 3.5: Missing Percent of Testing Data.................................................................... 33

Table 3.6: Training Data Interpolation Example ……...................................................... 37

Table 3.7: Testing Data Interpolation Example.................................................................. 38

Table 4.1: Traffic Conditions of the Simulated Network ….............................................. 49

Table 4.2: Performance Measure of the PCAB Method Comparing to Other Methods.. 52

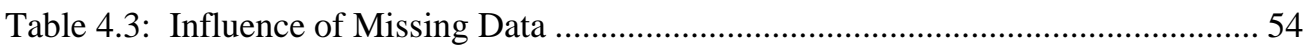

Table 4.4: Performance Measure of PLSB and PCAB Method with Time Mean Speed 56

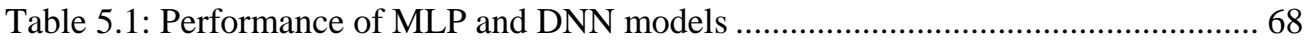

Table 5.2a: Prediction Results of Different DNN Models Compared to MLP Model ..... 69

Table 5.2b: Prediction Results of Different DNN Models Compared to MLP Model..... 69

Table 5.2c: Prediction Results of Different DNN Models Compared to MLP Model ..... 70

Table 5.2d: Prediction Results of Different DNN Models Compared to MLP Model ..... 70

Table 5.2e: Prediction Results of Different DNN Models Compared to MLP Model ..... 71

Table 5.3a: Model Performance with Varied Prediction Horizon for Daytime................. 72

Table 5.3b: Model Performance with Varied Prediction Horizon for Congestion Time.. 73 


\section{LIST OF FIGURES}

\section{FIGURE}

PAGE

Figure 1.1: Travel Time Provision in ATIS Framework .................................................... 1

Figure 1.2: Framework of Travel Time Prediction Modeling System................................. 4

Figure 2.1: Schematic Diagram for Speed Interpolation ............................................. 15

Figure 3.1: Map of the Study Corridor …………………............................................... 27

Figure 3.2: Schematic Locations of the Traffic Detectors ................................................ 28

Figure 3.3: Simple Imputation Method: Interpolation over Space and Time .................... 34

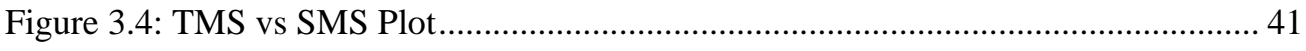

Figure 3.5: Total Residual with Varied Transition point of Regression Models.............. 42

Figure 4.1: Vehicle Trajectory Demonstration of Linear Speed Method ........................... 45

Figure 4.2: Flow Chart of the Route Travel Time Estimation Method ............................ 46

Figure 4.3: PCAB Estimated Travel Time Compared to Real Travel Time...................... 51

Figure 4.4: Experiment of Average Speed Method and PCAB Speed Method................. 52

Figure 4.5: Estimated Travel Time with Different Missing Data Percent......................... 55

Figure 4.6: Estimated Travel Time with Different Malfunction Detectors ....................... 56

Figure 4.7: Estimated Travel Time by PCAB Comparing to Real Travel Time ............... 57

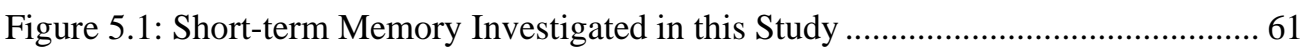

Figure 5.2: DNN Model Structure Investigated in this Study ........................................... 62

Figure 5.4: Travel Time Predicted by DNN Model in a Three-Day Period ...................... 65

Figure 5.5: MAPE of DNN, MLP and Instantaneous Estimation.....................................6 66

Figure 5.6: Performance Improvement of DNN Compared to MLP ................................. 67

Figure 5.7: MAPE with Varied Prediction Horizons ....................................................... 74 


\section{CHAPTER 1}

\section{INTRODUCTION}

\subsection{Background}

Travel time is a fundamental measure in transportation engineering because it is strongly related to the quality of service and can be easily comprehended by a wide variety of audience, including engineers, planners, administrators, and commuters. As a performance measure and decision-making variable, travel time information is becoming increasingly important for a variety of Advanced Traveler Information System (ATIS) and Advance Traffic Management System (ATMS) applications. The estimation and prediction of travel time has attracted significant interests of researchers as well as practitioners. The framework of travel time provision for ATIS is shown in Figure 1.1.

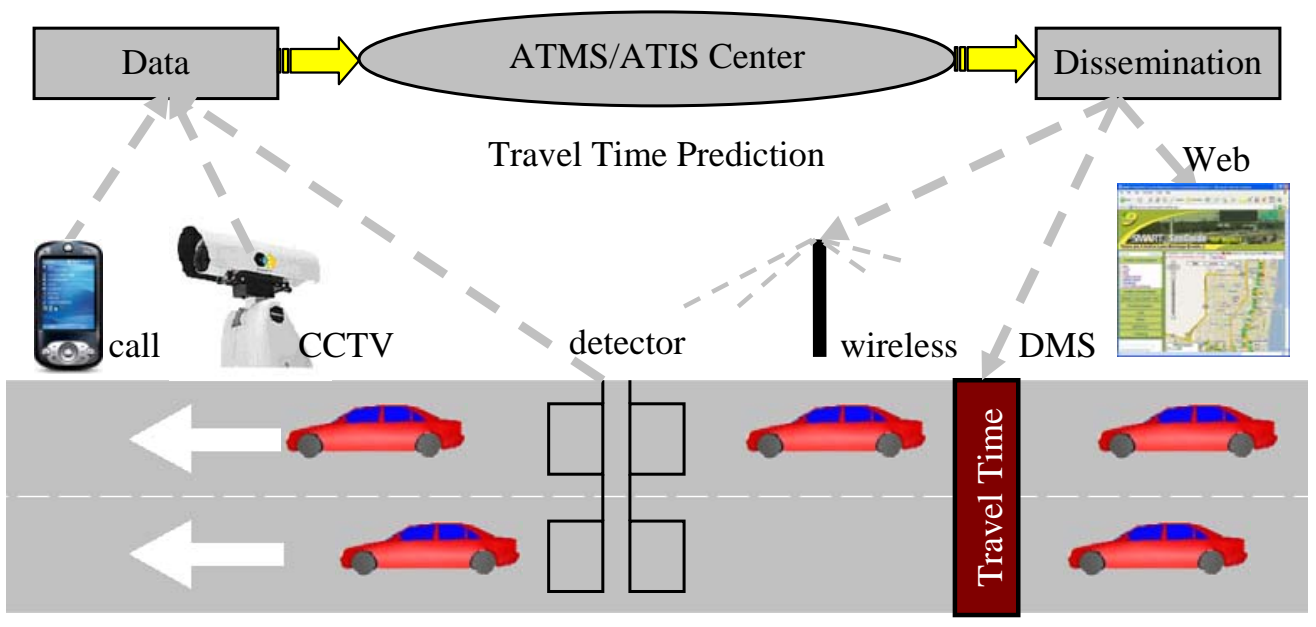

Figure 1.1: Travel Time Provision in ATIS Framework 
To be effective, the travel time information used for ATIS and ATMS applications should have two distinct characteristics: anticipative and dynamic. This is because every piece of information provided to users, to be useful, should yield predictions to the future and must be updated in real-time.

Providing transportation system operators and travelers with accurate traffic information allows them to make more informed decisions, yielding benefits for individual travelers and for the entire transportation system. Even in cases where no cost or time savings result from the provision of traffic information, the dissemination of information to travelers reduces uncertainty and increases comfort of travelers.

The increasing reliance on real-time travel time information indicates a need to measure travel time accurately and effectively. Essentially, the available travel time measurement techniques can be divided into two categories: direct methods and indirect methods. In the case of direct methods, the travel time information on corridor segments is collected directly from the field. These methods include probe vehicles tracking based on license plate matching, electronic vehicle tag matching, or Global Positioning System (GPS). In the indirect methods, the travel time of a corridor segment is estimated from traffic data like speed, flow, and/or occupancy collected by “point” traffic detectors such as microwave radar, infrared, loop detectors, and video image processing detectors.

While probe vehicle techniques are more accurate, they are more expensive and not as widely deployed as point detectors (Turner, 1996). On the other hand, freeways in most metropolitan areas in North America are or in the process of being instrumented with point traffic detectors. 
Recently, Florida Department of Transportation (FDOT) Districts have deployed above-ground, side-fire roadside microwave radar detectors as part of their freeway management systems. In general, these detectors are strategically placed approximately half mile apart. The traffic data such as speed, flow and occupancy are captured by detectors in a 20 second interval, then transferred to the Traffic Management Center (TMC) and recorded in the central software. In this study this traffic detector data will be used for travel time estimation and prediction.

\subsection{Problem Statement}

Most existing ATIS and ATMS use instantaneous travel times estimated based on the current measurements, assuming that traffic conditions will not change from the measured conditions in the near future. For more effective applications, it has been proposed that ATIS and ATMS should use travel times predicted for short-term future conditions rather than the instantaneous travel times measured or estimated for current conditions. The short term travel time prediction usually means to predict the current and near-future travel time up to 30 minutes and usually uses real-time and near-past traffic data. In some cases, historical data is also used as an additional input.

Providing accurate values for travel time is a complex and challenging problem. First, traffic detector data has inherent problems including missing and low quality data due to detector malfunctions. Thus, there is a need to check the quality of traffic detector data and use data cleaning methods to correct any discrepancies.

Second, as described above, travel times on roadway segments need be estimated from data collected using point traffic detectors. An effective method to estimate travel 
time from traffic detector data is needed for the success of the subsequent travel time prediction process.

Third, in most applications such as that in the FDOT Smart SunGuide software, instantaneous travel time is estimated with no prediction capability. Thus, these applications implicitly assume that the current traffic conditions remain constant until the vehicles finish their journeys. The problem with this approach is that as the vehicles travel along their routes traffic conditions change. Thus, there is a need for a method to more accurately and reliably predict travel times for the period that the vehicles are traveling on the road.

Figure 1.2 presents the framework of the travel time prediction processes and data required for travel time prediction modeling system considering the above discussion. The discussion presented in this section leads to the objectives of this study discussed in Section 1.3.

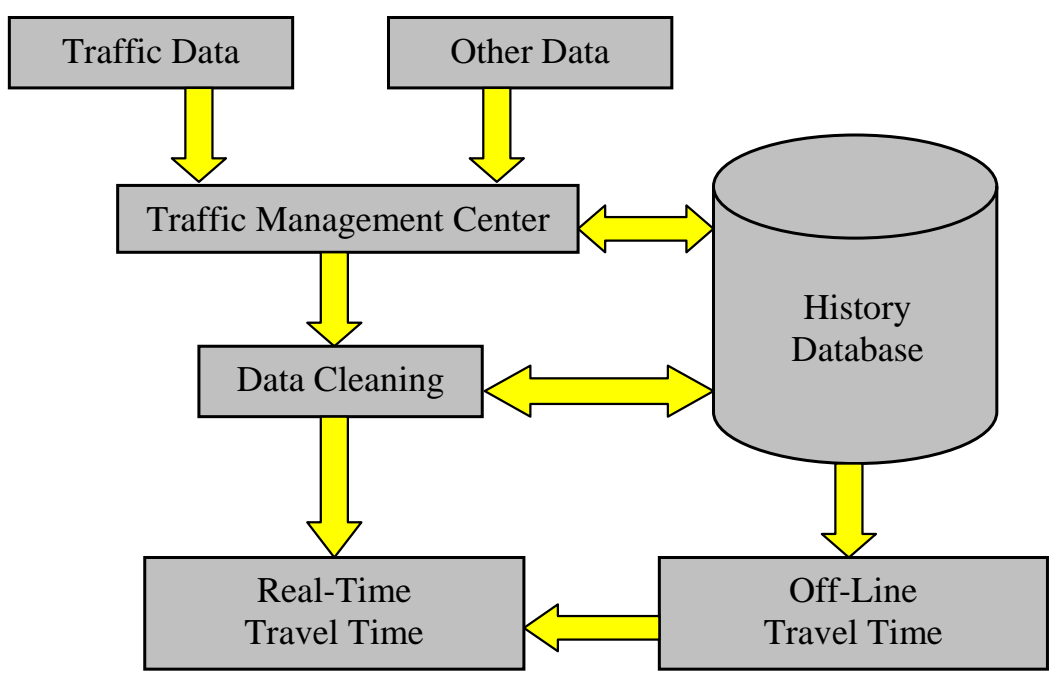

Figure 1.2: Framework of Travel Time Prediction Modeling System 


\subsection{Objectives and Scope}

This research aims to develop an improved method for the estimation and prediction of short-term freeway travel times from point traffic detector data. The method takes into account spatial and temporal variations in traffic data simultaneously. In other words, the method will be able to predict travel time based on traffic measures collected from different locations at the current and previous time periods.

The specific proposed research objectives are to:

1) identify data cleaning methods to deal with missing and erroneous traffic detector data and examine the effectiveness of the data cleaning methods,

2) identify and test a travel time estimation method along the link length based on point detector data,

3) develop a data-driven travel time prediction model and test the performance of the developed model, and

4) examine the influence of different model structures and parameters on the performance of the prediction model.

\subsection{Dissertation Organization}

This dissertation consists of six chapters: Chapter 1 introduces this dissertation research, puts forward the problem to be solved, and sets the goal and objectives to be achieved. Chapter 2 presents a review of the literature related to data cleaning, common travel time estimation and travel time prediction techniques and applications. Chapter 3 describes the traffic data, experiment environment, and the used data preprocessing procedures such as data imputation and data transformation. Chapter 4 presents an 
investigation of the segment travel time estimation method, which is required to produce the travel times used as inputs to the travel time prediction model. Chapter 5 presents the developed travel time prediction model and compares different dynamic neural networks topologies with varying parameters. Chapter 6 summarizes the major research results from this study, draws conclusions based on these results, and recommends issues to be considered in future research and application. 


\section{CHAPTER 2}

\section{LITERATURE REVIEW}

In the past few years, a large number of studies have been conducted which used a variety of mathematical methods to model traffic characteristics and produce travel time prediction.

\subsection{Classifications of Prediction Methods}

Below is a discussion of the classifications of the used methods based on the consideration of four basic factors: the scope of prediction, the prediction horizon and step, the input variables and the modeling approach.

\subsubsection{Scope of Application}

There are two broad categories of applications of travel time prediction: ATIS and ATMS. The implementation can also be categorized by the type of the highway facility including freeways, rural highways, and urban arterial streets. Predicting travel time has been proposed for use more in ATIS applications than in ATMS applications. Travel time prediction is more easily done on freeways and rural highways compared to urban arterial streets due to the difficulties in measuring travel time on urban arterials.

\subsubsection{Prediction Horizon and Step}

The prediction horizon, which defines the time window after which the prediction is made, is usually set in the range of $0-15$ minutes. The prediction horizon is used to account for situations in which the vehicles receiving the information will arrive at the 
point where the prediction is required at some time in the future. This may be, for example, the cases of information provided by traveler information web sites, hand-held devices, and in-vehicle devices. In these cases, the prediction will need to be made starting at the time that these vehicles are expected to arrive at the point of prediction. With the prediction horizon of zero minutes, the model predicts the travel time starting at the current time. The use of a prediction horizon of zero is appropriate, for example, for the prediction of travel time displayed on Dynamic Message Signs (DMS) that provide travel time information to vehicles passing by the DMS. Ishak and Al-Deek (2002) concluded that the prediction accuracy degrades as the predicting horizon increases. Also, different prediction horizons yield different modeling approaches and methodologies.

The prediction rolling step defines the temporal resolution of input data in terms of the time interval, at which the historical travel time data is aggregated for use in the analysis. Previous studies have found that there is a decrease in the prediction accuracy due to the strong variability of traffic parameters when examined in short time intervals (e.g. 30 seconds). For this reason, researchers have used aggregated data in 1-5 minutes intervals to obtain more stable traffic characteristics (Park and Rilett, 1998; Van Lint et al. 2002). The use of coarser levels of data aggregation (10 minutes or more) leads to reduced fluctuations in the data even further, and results in the loss of valuable real-time information. Defining the appropriate data resolution is a very important issue because it affects the quality of information that is used as input to the travel time estimation and prediction models. 


\subsubsection{Input Variables}

Advanced traffic surveillance techniques such as probe vehicle techniques including automatic vehicle location (AVL) and automatic vehicle identification (AVI) systems allow direct travel time data collection (Chen and Chien, 2001; Chien and Kuchipudi, 2003). However, the travel time measurements for a route from an origin to a destination become available only after a vehicle has finished the entire route with these technologies. This makes this measurement not appropriate for online travel time prediction purposes because of the long time required to complete the paths. Usually, however, the travel time is measured using probe vehicles at a short section level (one to two miles). The travel time in many cases is required for short segments such as between DMS locations.

Another type of measurements that are more widely available is traffic point measurements that measure traffic parameters such as speed, flow, and occupancy using traffic detectors. These are normally aggregated at a 20 second to one minute interval. The travel time can be estimated from these detector data when direct measurement of travel time is not available, as discussed later in this dissertation.

\subsubsection{Modeling Approach}

Travel time prediction approaches can be categorized into model-based and datadriven approaches.

Model based travel time prediction methods predict traffic conditions based on traffic flow theory or real-time traffic simulation. Given the wide body of research on traffic flow theory in the past decades, this choice seems most appropriate. However, it 
inherently forces the modeler to predict the traffic conditions at the boundaries of the model used (traffic flow at origins/destinations and ramps, capacity restrictions, etc). Simulation models have been applied in real-time to predicting travel time using a rolling horizon process. Examples of this approach are the use of DYNAMIT (Ben-Akiva et al. 2003), METANET (Smulders et al. 1999), DYNASMART (Hu, 2001), and CORSIM (Liu, et al. 2006b). The predictive quality of the model-based travel time prediction is strongly influenced by the quality of the input data, the used models, and the model calibration. The accuracy of the model's output, even if the model reproduces traffic patterns very accurately, can only be as good as the predictive accuracy of its inputs. Moreover, setting up real-time traffic flow models for online short term travel time prediction on the large number of routes typically encountered in a transportation network would require significant modeling effort in terms of design, implementation, calibration, and maintenance.

Data-driven methods predict travel times based on current and past real-world detector data, without explicitly considering the physical traffic processes considered by the model-based methods. For data-driven methods, the used method, the quality of data, and the parameters used in the development and implementation of the method influence the quality of prediction. There are many successful applications reported on data-driven approaches for short term freeway travel time prediction.

The availability of advanced traffic data collection, storage and maintenance system is required in order to apply data-driven approaches. If no such system is available, only the model-based approaches can be used. A densely spaced local data collection system enables a much more accurate and reliable applications of data-driven 
travel time prediction models. Further discussion of this approach is presented later in this chapter.

\subsection{Data Preprocessing}

Traffic detector data has inherent problems including missing and low quality data due to detector malfunctions. In addition, point traffic detector can only collect the time mean speed which is not suitable to derive travel time as discussed later. Data preprocessing is usually needed before travel time estimation and prediction. This preprocessing includes data cleaning, imputation, and transformation as discussed in the following subsection.

\subsubsection{Data Cleaning and Imputation}

Input failure is the occurrence of erroneous or missing data in the input vector. This happens when a measurement device produces data that is dubbed unreliable, or when it produces no data at all. Input failure can be classified into two types: random failure that occurs due to, for example, temporary power or communication failures of the freeway monitoring system; and structure failure that occurs mainly due to physical damage or maintenance blockage to the detectors or other associated roadside equipment. In real life, a mix of these input failure types will occur.

Smith and Demetsky (1997) underlined the inability of Auto Regressive Integrated Moving Average Model (ARIMA) travel time prediction models to deal with missing values. Later, Chen et al. (2001) commented on the effects of missing values in a comparative study between an ARIMA model and the neural networks (NNs) approach. 
The findings showed that the ARIMA models were more sensitive in dealing with missing values and various types of imputation techniques than NNs models.

Although non-parametric techniques such as NNs are more robust concerning the missing values given that it is designed and trained properly (Bishop, 1995), missing data is still expected to affect the accuracy of NNs training, significantly. The use of an effectiveness of data cleaning method is crucial.

As Chen et al. (2001) underlined, if the prediction models do not have the ability to deal with false or missing values, it is up to the practitioner to select the proper datacleaning technique. This stage of traffic data preparation is very important, particularly for conventional statistical approaches.

In general, three approaches have been used for replacing missing values: null replacement, simple imputation, and advanced imputation. Null replacement refers to leaving the data as is or replacing missing data with some default values. In this case, the model that uses the data has to be robust enough to deal with the missing data problems.

Simple imputation involves replacing missing values by some statistical measures. These could include: the sample mean, median or other descriptive statistic (Chen et al. 2001). In practice, this is the most commonly used approach to remedy the missing and erroneous data problem. Schafer (1997) shows that simple imputation schemes tend to change the covariance structure of the input-data and may induce bias. Despite the clear theoretical shortcomings of simple imputation schemes as discussed from different perspectives by Armitage and Lo (1994), the results presented by Chen et al. (2001) indicate that simple imputation combined with NNs based traffic predictor does produce accurate traffic predictions, even when up to $30 \%$ of the input data is missing. 
Advanced imputation is in essence a special case of simple imputation. In this case missing values are replaced by procedures which can be treated as a prediction submodel, rather than a simple statistical method. Examples include the use of traffic flow simulation models and Kalman Filters. In the DACCORD project (Thijs et al. 1998a; Thijs et al. 1998b; Thijs et al. 1999), two imputation methods were implemented and extensively tested on three different test sites. The first method was based on a Kalman filter and the second based on a cross-correlation algorithm. Haj-Salem and Lebacque (2002) used a data cleaning strategy based on a first order (linear) traffic flow model, producing even better results than the methods applied in DACCORD. Traffic flow model-based imputation schemes seem to be the most appropriate data cleaning tool for traffic prediction purposes, because they address the spatial and temporal characteristics of traffic processes. On the down side, however, they require much more modeling effort than simple statistical methods such as interpolation or smoothing technique. Secondly, a traffic flow simulation model is parameterized and requires calibration. Another disadvantage to the use of model-based imputation is the computational expense which is particularly relevant in real-time operations of the models.

\subsubsection{Data Transformation}

Freeway ITS applications usually include traffic detectors (microwave radar, infrared, inductive loop, or video based systems) that measure the spot (time mean) speed, flow and occupancy. However, travel time prediction requires predicting of link (space mean) speed along highway segments. Thus, transformation from time mean speed to space mean speed is required. 
Wang and Nihan (2000) estimated space mean speed from flow and occupancy based on traffic flow theory. Rakha and Zhang (2005) proposed a formulation for estimating space-mean speeds with a small margin of error (within 0 to 1 percent) given time mean speed and the variance. Garber and Hoel (2002) described a linear relationship between time-mean and space-mean speeds. Van Lint (2004) found that in congested conditions the speed variance is nearly constant, while in free-flow conditions, variance is a steeply increasing function of the mean speed. He presented different linear models for these two traffic conditions separated by a transition point.

\subsection{Travel Time Estimation}

As stated in section 2.1.3, for most applications, travel time is not measured directly as in the case with the probe vehicle methods, but measured indirectly by traffic detectors (point measurements). For data-driven models based on detector data, the travel time estimation pertains to reconstructing travel times of realized trips from traffic data recorded by detectors. An accurate travel time estimation method is of vital importance to compile databases of estimated travel times with which subsequent travel time prediction models can be calibrated and validated. The different methods reported for the estimation of travel time based on point measurements can be broadly divided into two main categories: trajectory methods and traffic flow model based methods.

\subsubsection{Trajectory Methods}

A trajectory method is a speed based method that requires the conversion of the inputs from spot time mean speed to an estimate of space mean speed for better 
performance achievement. This conversion has been discussed in Section 2.2.2 of this document.

The Travel Time Data Collection Handbook (1998) reports the trajectory techniques as the simplest and most widely accepted method for the estimation of travel time from traffic detector data. The speed is assumed to be constant for the small distance between the measurement points, usually the distance between the two detector stations (approximately 0.5 miles). Since the distance between the two detectors is known a priori, the travel time is calculated as the distance divided by the speed (Dailey 1997; Lindveld et al. 2000; Cortes et al. 2002; Van Lint and Van der Zijpp, 2003). Thus, the trajectory methods assume that the point estimates of speed are representative of the average speed between the adjacent loop detectors.

The three different extrapolation approaches normally adopted at present are explained below with the help of Figure 2.1.

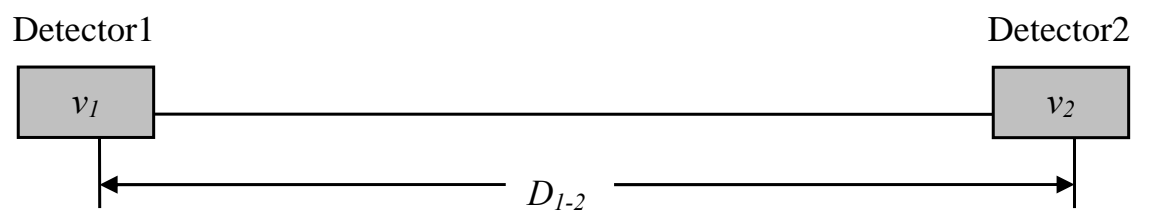

Figure 2.1: Schematic Diagram for Speed Interpolation

1) Half distance method: the speed measured by a detector is applicable to half the distance on both sides.

$$
T_{1-2}=\frac{1}{2}\left(\frac{D_{1-2}}{v_{1}}+\frac{D_{1-2}}{v_{2}}\right)
$$

2) Average speed method: the speed is assumed to be the average speeds measured by detectors 1 and 2 . 


$$
T_{1-2}=\frac{D_{1-2}}{\left(v_{1}+v_{2}\right) / 2}
$$

3) Minimum speed method: the speed is assumed to be the minimum speeds measured by detectors 1 and 2 .

$$
T_{1-2}=\frac{D_{1-2}}{\operatorname{Min}\left(v_{1}, v_{2}\right)}
$$

where $v_{1}$ and $v_{2}$ are the space mean speeds calculated based on the measurements at detector 1 and 2 respectively, $D_{1-2}$ is the distance between detectors 1 and detector 2, and $T_{1-2}$ is the travel time from detector 1 to detector 2.

The main disadvantage of the constant speed trajectory methods is the decreasing performance with increasing traffic congestion (Lindveld et al. 2000). Also, the assumption of constant speeds between the detection points holds true only at low to moderate volume conditions, where the variability in the flow is lower (Coifman, 2002). At high volume conditions due to the variation of speed, the assumption of a constant speed no longer holds true even for a small section of road. Thus, the error in the travel time results calculated using these methods tends to increase during congested periods.

Van Lint (2003) proposed an improved travel time estimation algorithm called Piecewise Linear Speed Based (PLSB) algorithm. With this algorithm, the section speed changes along the longitudinal position and is assumed to be a linear interpolation between the observed speed at the upstream and downstream locations. Tested with simulation data, Van Lint's study showed that the root mean squared error (RMSE) of the PLSB method is about half of the constant speed trajectory method which is the half distance method. Further discussion of the PLSB method is presented in Section 3.2. 


\subsubsection{Traffic Flow Theory Method}

Theoretical models also have been developed for the estimation of travel time from loop detector data based on traffic flow theory. The advantage of these models is that since they are based on the traffic flow theory, they can capture the dynamic characteristics of the traffic process. One approach used the principle of conservation of vehicles by comparing the inflow of a section during previous time period with its outflow during the current time period. This approach was used by Nam and Drew (1996, 1998, 1999) who presented a macroscopic model for estimating freeway travel time in real-time directly from flow measurements based on the area between the cumulative volume curves from loop detectors at either end of the link. Petty et al. (1998) suggested a model for estimating travel time directly from flow and occupancy data, based on the assumption that the vehicles that arrive at an upstream point during a given interval of time have a common probability distribution of travel times to a downstream point. Coifman (2002) utilized the linear approximation of the flow density relationship to estimate travel time from dual-loop detector data assuming constant shockwave speed. The results were reported as satisfactory except at the transition periods from congested to un-congested conditions and vice versa.

Most of the theoretical studies used for travel time estimation from detector data give satisfactory results for specific conditions only. For instance, some of the models performed well for normal-flow conditions only (Nam and Drew, 1996; Hoogendoorn, 2000), while other models are applicable for congested traffic flow conditions only (Nam and Drew, 1998). Another important premise for the traffic flow based models is that all the values of the input and output flows around the boundary of the study road section are 
available, which in most cases is not the case since most of the ramp flows are not monitored by traffic detectors.

\subsection{Travel Time Prediction}

The effectiveness of the ATIS depends on the accuracy and timeliness of traffic information provided. Predicted travel time for future is more beneficial than current information since traffic conditions may change significantly before travelers complete their journeys.

There is a need for an effective methodology for predicting travel times. Several short-term travel time prediction methods have been derived. Based on the mathematical methodology used, the methods can be broadly classified into three categories: regression methods, time series methods, and NNs methods. Some methods use historical data in addition to real-time data. The following sections include an overview of the methods reported in the literature.

\subsubsection{Methods Based on Historical Data}

The methods that use historical data either alone or combined with real-time data are rational because historical profiles can represent the average traffic characteristics over days when the future traffic has a similar profile. Thus, a historical average can be used for predicting the future values. This approach involves the creation of a historical data profile and then classify days into day types with similar profile. When not combined with real-time data, this approach is relatively easy to implement and fast in terms of computation speed. Also, this method can be valuable in the development of prediction models since they explain a substantial amount of the variation in traffic over 
time periods and days. However, the value of static prediction is limited because of its implicit assumption that the projected traffic remains constant for different days of the same type. Commuters in general have a good knowledge of average travel time under usual traffic conditions. They are more interested in knowing travel time under not-socommon conditions, when average values are not representative of the current or future traffic conditions. The historical data method performs reasonably well under normal conditions. However, it can misrepresent the conditions when the traffic is abnormal.

The Advanced Driver and Vehicle Advisory Navigation Concept (ADVANCE) project in the Chicago metropolitan area used a combination of historical and instantaneous data for their travel time prediction model (Tarko and Rouphail, 1993; Boyce et al. 1993). Seki (1995) used historical data after correcting them by type of day for prediction of travel time. Manfredi et al. (1998) developed a prediction system as part of the DACCORD project mentioned earlier, where the prediction was based on historical and instantaneous data. Zhang and Rice (2003) used a varying coefficients linear model with past instantaneous travel time to predict the future travel time. Rice and Zwet (2004) investigated the combined instantaneous and historical travel time data, using statistical methods such as principle component analysis and windowed nearest neighbor. Chien and Kuchipudi (2003) explored the travel time prediction problem using travel time data directly collected through roadside terminals and found that using aggregated historical data in the same weekday (up to four weekdays) combined with real-time data have comparable results with using real-time data from previous time intervals. 


\subsubsection{Regression Methods}

Kwon et al. (2000) presented an approach to predicting travel time using linear regression with a stepwise variable selection method using flow and occupancy data from loop detectors and historical travel time information. Rice and Zwet (2004), Zhang and Rice (2003) and Sun et al. (2007) proposed freeway travel time prediction methods using linear regression models with coefficients that varies with the time of day.

\subsubsection{Time Series Methods}

Researchers have used state-space models that belong to the multivariate family of time series models. The time series method of travel time prediction involves the examination of historical data, extracting essential data characteristics, and effectively projecting these characteristics into the future to predict the travel time in future time steps from the travel time (and possibly other traffic characteristics) at the current and previous time steps.

ARIMA was first introduced in traffic forecasting by Ahmed and Cook (1979) and Levin and Tsao (1980) as an alternative approach to model the stochastic nature of traffic. Oda (1990) adopted an auto-regressive model for the prediction of travel time. Saito and Watanabe (1995) developed a system for predicting the travel time for 60 minutes in the future using an auto regression model based on the change in traffic conditions for the previous 30 minutes. Iwasaki and Shirao (1996) discussed a short-term prediction scheme of travel time a long a section of a motorway using an auto regressive method. The parameters of the prediction model were identified by adapting an extended Kalman filtering method. D’Angelo and Al-Deek (1999) and Ishak and Al-Deek (2002) 
implemented models that used nonlinear time series with multi-fractal analysis for the prediction of travel time.

If broadly defined, the time series class can also include the Kalman filter method, which has the advantage of updating the selected state variables continuously. Chen and Chien (2001), Chien and Kuchipudi (2003), and Nanthawichit et al. (2003) used Kalman filtering for travel time prediction.

\subsubsection{Neural Networks Methods}

The real power of NNs is not only their proven ability to provide good predictions but also their overall performance and robustness in traffic modeling. Some of their advantages can be summarized as follows:

1) NNs can produce accurate multiple step-ahead prediction.

2) NNs have been tested with significant success in modeling complex temporal and spatial relationships lying in datasets from different fields including transportation engineering.

3) NNs is capable of modeling highly non-linear relationships in a multivariate setting (Zhang et al. 1998).

NNs applications to short-term traffic prediction extend from the simple multilayer perceptron (MLP) (Smith and Demetsky, 1994; Park and Rilett, 1999; Zhang, 2000; Huisken and Van Berkum, 2003; Innamaa, 2005) to more complex structures such as MLP with a learning rule based on a Kalman filter (Vythoulkas, 1993); modular neural networks (Park and Rilett, 1998); radial basis neural networks (Park et al. 1998); spectral basis neural networks (Park et al. 1999; Rilett and Park, 2001); time-delayed neural 
networks (TDNN) (Yun et al. 1998; Abdulhai et al. 1999; Dia, 2001; Lingras et al. 2002; Ishak et al. 2003); and state-space neural networks (SSNN) (Van Lint et al. 2002; Van Lint et al. 2005; Van Lint, 2006; Liu, et al. 2006a, Singh, and Abu-Lebdeh, 2007).

The support vector machine (SVM) method can also be classified as a NNs method and has been investigated for travel time prediction by Wu et al. (2003) and Vanajakshi (2004).

Another approach for traffic prediction is the hybrid methods that use a mixture of methods to construct a smaller (reduced dimensionality) and more efficient network. This concept was proven to be applicable in cases where clustering techniques were first applied to the available data. For example, the ATHENA model (Danech-Pajouh and Aron, 1991) is a layered statistical approach that adopted a clustering technique to group the data and assign each cluster to a linear regression model. Van der Voort et al. (1996) introduced a different hybrid model that combined Kohonen maps with ARIMA models to forecast traffic flow, which improved the performance compare to simple ARIMA model and MLP model. Later Chen et al. (2001) presented two hybrid neural networks approaches, a Self-Organizing Map (SOM) combined with ARIMA model and a SOM/MLP model, and found that the SOM/MLP approach achieves superior results.

New interest in hybrid methods arises from the use of fuzzy logic and genetic algorithms. Palacharla and Nelson (1999) first applied a fuzzy-neural network for travel time prediction. Yin et al. (2002) developed a fuzzy-neural model that consisted of two modules: a gate network for classification of the input data into a number clusters using fuzzy approach and an expert network for specifying input-output relationships based on the conventional neural networks approach. The model performed better and in less 
computational time than a simple neural networks model. A recent application of neuralfuzzy systems by Ishak and Alecsandru (2003) applied an adaptive neural-fuzzy inference system to reduce the dimensionality of the input space.

Genetic algorithms (GA) have also been combined with NN. The GA approach has the natural propensity of searching through cast and complex solution spaces that encompass a great number of local minima. Abdhulai et al. (1999) developed a combined GA and time-delayed neural networks. In this case, the GA was used to optimize the look-back interval of the network. Lingras et al. (2002) applied timedelayed neural networks with embedded GA to maximize the statistical correlation for selecting connections between the input and hidden layers. The hybrid application of GA could be extremely important in several optimization issues concerning the internal architecture of neural networks.

\subsection{Summary}

Chapter 2 has presented a review of the literature of traffic travel time estimation and prediction and related input data issues. Previous studies illustrated that NNs can provide an accurate and robust approach to travel time prediction. NNs' applications to travel time prediction have included several types of NNs for dynamic travel time estimation. It was reported in these studies that NNs can out-perform other travel time prediction techniques, including the regular time series and Kalman filtering approaches. Based on the previous literature, the SSNN topology has been used for travel time prediction. The TDNN topology has been investigated for traffic volume prediction, but not for travel time prediction. Both of these types of networks are sub-classes of 
Dynamic Neural Networks (DNN), which takes into account spatial and temporal information about traffic conditions simultaneously which makes them particularly suitable for travel time prediction.

This research will investigate the ability of a number of DNN topologies with different parameters settings to predict travel time and compares the performance of the prediction with a simple MLP neural network. Other associated processes such as data cleaning and transformation and travel time estimation will also be applied and assessed. 


\section{CHAPTER 3}

\section{DATA ACQUISITION AND PREPROCESSING}

\subsection{Introduction}

This Chapter discusses the data acquisition and data preprocessing step of this research. As described in previous chapters, traffic detector data has inherent problems including missing and low quality data due to detector malfunctions. Thus, there is a need to check the quality of traffic detector data and use data cleaning methods to correct any discrepancies which is an important step required for the modeling of travel time prediction. This chapter discussed the source of the data used in this study and the data preprocessing step of the travel time estimation.

The traffic data used in this study was obtained from the Florida Department of Transportation (FDOT) District 4 traffic detection system deployed along the I-95 corridor and stored in the traffic management center (TMC) database. FDOT District 4 has recently deployed above-ground, side-fire roadside microwave radar detectors along I-95 as part of their freeway management system. In general, these detectors are strategically placed approximately half mile apart. Traffic data such as speed, volume, and occupancy are captured by the traffic detectors at 20 seconds interval, then transferred to the TMC and stored in the central database. The database records including the timestamp, detector ID, lane ID (varied from 4 up to 6 lanes), time mean speed, occupancy, and volume of the past 20 seconds before the TMC receives the data.

The three basic traffic flow parameters (volume, occupancy, and speed) are directly related to each other as proved by the traffic flow theory. Any one of these 
parameters can be derived from the other two parameters. Thus, in this study, to avoid redundant information input into the model, only speed and volume data is used for travel time prediction modeling and analysis. Table 3.1 lists a sample of recorded traffic detector data used for in this study.

Table 3.1: Example of Traffic Detector Data Records

\begin{tabular}{|c|c|c|c|c|c|}
\hline ID & timestamp & detector id & lane id & speed (mph) & volume \\
\hline 294459 & 6:00:02 & 95SB019.5 & 95SB019.5-Lanes-lane1 & 85 & 3 \\
\hline 294458 & 6:00:02 & 95SB019.5 & 95SB019.5-Lanes-lane2 & 71 & 1 \\
\hline 294457 & 6:00:02 & 95SB019.5 & 95SB019.5-Lanes-lane3 & 68 & 2 \\
\hline 294456 & 6:00:02 & 95SB019.5 & 95SB019.5-Lanes-lane4 & 63 & 3 \\
\hline 294455 & 6:00:02 & 95SB019.5 & 95SB019.5-Lanes-lane5 & 64 & 1 \\
\hline 294410 & 6:00:02 & 95SB021.0 & 95SB021.0-Lanes-lane1 & 87 & 8 \\
\hline 294411 & 6:00:02 & 95SB021.0 & 95SB021.0-Lanes-lane2 & 84 & 5 \\
\hline 294412 & 6:00:02 & 95SB021.0 & 95SB021.0-Lanes-lane3 & 74 & 5 \\
\hline 294413 & 6:00:02 & 95SB021.0 & 95SB021.0-Lanes-lane4 & 80 & 4 \\
\hline 294414 & 6:00:02 & 95SB021.0 & 95SB021.0-Lanes-lane5 & 75 & 3 \\
\hline
\end{tabular}

Originally the traffic detector data obtained from the TMC was stored in an individual text file for each day. For the convenience of data manipulation, the data was imported to a SQL database and merged together. Based on a quick preview of the data, it was found that the data of the three months of June, July and August in 2007 was more complete than the other months. Thus, the three months data was used for the travel time prediction modeling. June and July data was used for NNs model training and August data for NNs model testing. Further, the detector data of the I-95 corridor between the I595 interchange and the Broward/Miami-Dade County line (about 8.25 miles) was found to be more complete than the other segments. Thus, this segment was chosen for use in this research. Figure 3.1 shows the map of the study corridor. 


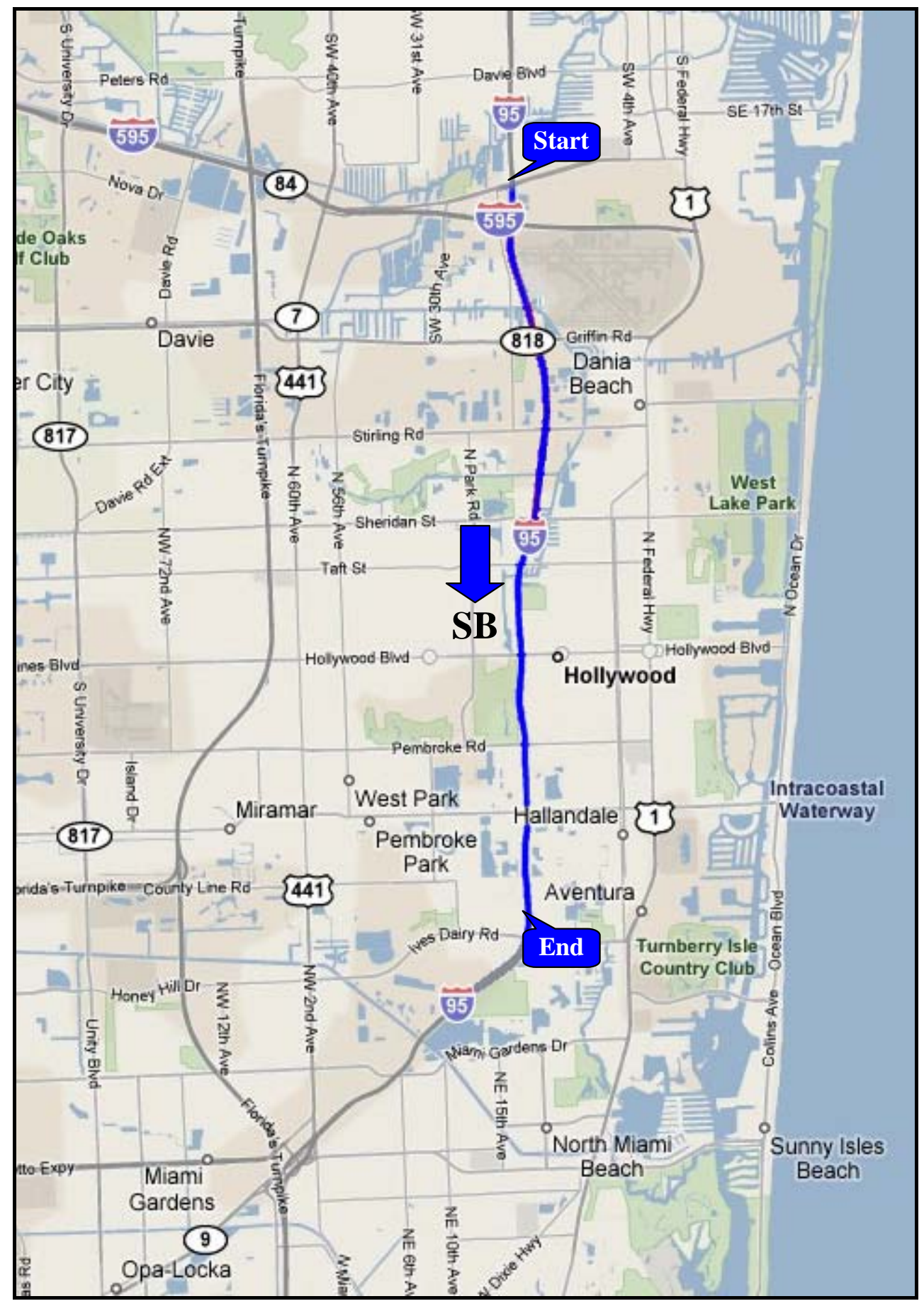

Figure 3.1: Map of the Study Corridor 
In this study, traffic detector data from 11 out of the total of 16 detectors on the study section were used for travel time prediction modeling due to the detector malfunction problems that are associated with the remaining five detectors. It is believed that the malfunction problems occur because the detectors were just installed at the time of the data collection and these problems had not been fixed by the contractor that installed the detectors. The specific geometrical deployment of the 11 detectors is shown in Figure 3.2. D1 to D11 in Figure 3.2 refer to the locations of the 11 detectors. Also the Figure shows the locations of the on and off ramps.

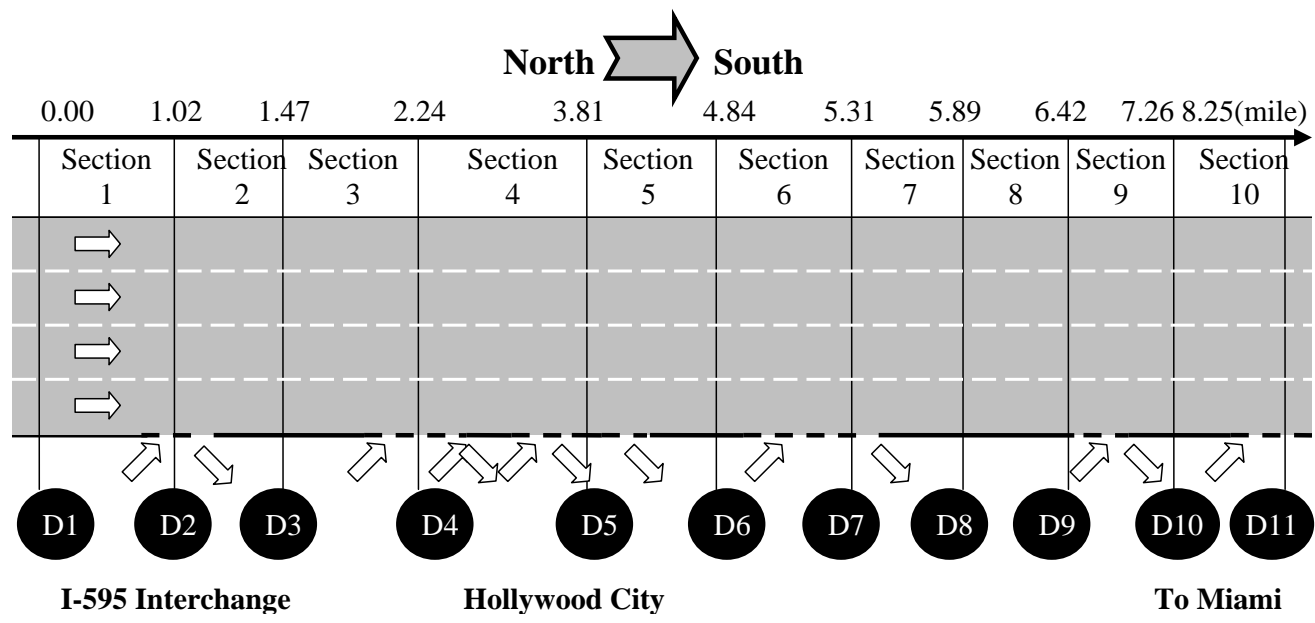

Figure 3.2: Schematic Locations of the Traffic Detectors

Another data source is the incident data which is gathered from the freeway incident management program of FDOT District 4 in Fort Lauderdale, FL, and stored in a comprehensive database called the Systems Management for Advanced Roadway Technologies (SMART) SunGuide database. The stored data attributes include timestamps of the activities for all agencies involved, the tracking of lane and shoulder closures and clearances, incident location information, and other incident and incident 
management attributes. A sample incident data is shown in Table 3.2. This data was used to confirm the reasons for non-recurrent peaking congestion during the analysis period.

Table 3.2: Sample Incident Data

\begin{tabular}{|c|c|c|c|c|c|c|c|}
\hline ID & Type & $\begin{array}{c}\text { Report } \\
\text { Time }\end{array}$ & Location & Milepost & $\begin{array}{c}\text { \# Lanes } \\
\text { Closed }\end{array}$ & $\begin{array}{c}\text { Response } \\
\text { Time }\end{array}$ & $\begin{array}{c}\text { Clearance } \\
\text { Time }\end{array}$ \\
\hline 367497 & Disabled Vehicle & August 14 & Exit 23 & 6.188 & 1 & 9 & 22 \\
\hline 367591 & Accident & August 14 & Exit 18 & 0.782 & 1 & 12 & 47 \\
\hline 369840 & Accident & August 21 & Exit 21 & 4.414 & 1 & 0 & 74 \\
\hline 371992 & Accident & August 27 & Exit 21 & 4.120 & 1 & 1 & 120 \\
\hline 372116 & Disabled Vehicle & August 27 & Exit 21 & 3.860 & 1 & 15 & 41 \\
\hline
\end{tabular}

Note: Time unit is minute.

\subsection{Data Preparation}

After the data was imported to the SQL database, a program was written in $\mathrm{C}++$ to manipulate the data in preparation for future use. In the original data table, each data row has a timestamp attribute which indicates the time at which the data was received at the central database. Because of difference in the detector clock setup and possible small delays in the transformation of the data from different detector locations, there was a need to synchronize the data to a common base timestamp. Different segments on the study corridor have different number of lanes varying from 4 to 6 lanes. The traffic data is collected by the detectors and stored in the database for each lane separately. In this study, the traffic data for all lanes was aggregated together for each detector location. For the convenience of future data manipulation the traffic detector data was then rearranged according to location along the route to be used as the database for further analysis as shown in Table 3.3. 
Table 3.3: Rearranged Traffic Detector Data Table

\begin{tabular}{|c|c|c|c|c|c|c|c|c|c|c|c|c|c|c|c|c|c|c|c|c|c|c|c|}
\hline \multirow{2}{*}{ ID } & \multirow{2}{*}{ timestamp } & \multicolumn{2}{|c|}{ Det.1 } & \multicolumn{2}{|c|}{ Det.2 } & \multicolumn{2}{|c|}{ Det.3 } & \multicolumn{2}{|c|}{ Det.4 } & \multicolumn{2}{|c|}{ Det.5 } & \multicolumn{2}{|c|}{ Det.6 } & \multicolumn{2}{|c|}{ Det.7 } & \multicolumn{2}{|c|}{ Det.8 } & \multicolumn{2}{|c|}{ Det.9 } & \multicolumn{2}{|c|}{ Det.10 } & \multicolumn{2}{|c|}{ Det.11 } \\
\hline & & $\mathrm{S}$ & $\mathbf{V}$ & $\mathrm{S}$ & $\mathbf{V}$ & $\mathrm{S}$ & $\mathbf{V}$ & S & $\mathbf{V}$ & $\mathrm{S}$ & $\mathbf{V}$ & S & $\mathbf{V}$ & S & $\mathbf{V}$ & S & $\mathbf{V}$ & $\mathrm{S}$ & $\mathbf{V}$ & $\mathrm{S}$ & $\mathbf{V}$ & $\mathrm{S}$ & $\mathbf{V}$ \\
\hline 1 & 06:00:00 & 70 & 26 & 72 & 18 & 72 & 20 & 75 & 13 & 73 & 33 & 66 & 36 & 68 & 29 & 79 & 19 & 74 & 19 & 73 & 24 & 66 & 23 \\
\hline 2 & 06:00:20 & 69 & 23 & 77 & 16 & 68 & 16 & 74 & 26 & 73 & 35 & 70 & 25 & 69 & 20 & 80 & 26 & 75 & 11 & 70 & 16 & 67 & 12 \\
\hline 3 & 06:00:40 & 70 & 20 & 77 & 19 & 68 & 27 & 70 & 35 & 72 & 25 & 71 & 21 & 72 & 33 & 83 & 29 & 72 & 17 & 69 & 20 & 66 & 13 \\
\hline 4 & 06:01:00 & 71 & 29 & 81 & 17 & 63 & 34 & 69 & 31 & 70 & 19 & 74 & 30 & 72 & 12 & 81 & 22 & 71 & 13 & 65 & 15 & 66 & 26 \\
\hline 5 & 06:01:20 & 75 & 21 & 76 & 25 & 68 & 52 & 70 & 23 & 71 & 27 & 73 & 26 & 74 & 21 & 80 & 34 & 64 & 14 & 70 & 13 & 67 & 10 \\
\hline 6 & 06:01:40 & 76 & 18 & 80 & 35 & 67 & 25 & 71 & 20 & 74 & 21 & 72 & 36 & 72 & 30 & 80 & 16 & 72 & 13 & 71 & 18 & 70 & 13 \\
\hline 7 & 06:02:00 & 78 & 23 & 75 & 44 & 68 & 27 & 74 & 25 & 71 & 31 & 72 & 25 & 72 & 15 & 76 & 31 & 72 & 15 & 72 & 12 & 69 & 16 \\
\hline 8 & 06:02:20 & 75 & 37 & 74 & 27 & 73 & 30 & 77 & 26 & 72 & 23 & 75 & 24 & 72 & 24 & 74 & 23 & 74 & 12 & 70 & 10 & 68 & 18 \\
\hline 9 & $06: 02: 40$ & 72 & 39 & 72 & 15 & 73 & 27 & 73 & 31 & 77 & 22 & 76 & 32 & 70 & 16 & 73 & 30 & 73 & 6 & 75 & 18 & 70 & 18 \\
\hline 10 & 06:03:00 & 70 & 39 & 73 & 23 & 75 & 32 & 76 & 29 & 78 & 27 & 77 & 14 & 66 & 13 & 75 & 25 & 72 & 16 & 75 & 32 & 69 & 10 \\
\hline 11 & 06:03:20 & 70 & 22 & 76 & 26 & 73 & 28 & 76 & 22 & 74 & 15 & 75 & 30 & 68 & 17 & 72 & 9 & 71 & 13 & 76 & 11 & 67 & 23 \\
\hline 12 & 06:03:40 & 72 & 20 & 74 & 26 & 73 & 32 & 78 & 23 & 74 & 26 & 73 & 19 & 66 & 29 & 75 & 22 & 69 & 14 & 73 & 16 & 66 & 13 \\
\hline 13 & 06:04:00 & 68 & 37 & 70 & 30 & 70 & 28 & 78 & 15 & 72 & 17 & 73 & 23 & 69 & 37 & 79 & 22 & 71 & 18 & 78 & 19 & 65 & 16 \\
\hline 14 & 06:04:20 & 72 & 24 & 70 & 28 & 72 & 19 & 75 & 28 & 71 & 21 & 72 & 18 & 69 & 15 & 75 & 33 & 71 & 9 & 71 & 13 & 67 & 26 \\
\hline 15 & 06:04:40 & 70 & 31 & 75 & 26 & 69 & 27 & 72 & 15 & 72 & 15 & 69 & 29 & 72 & 21 & 78 & 15 & 70 & 9 & 74 & 20 & 68 & 20 \\
\hline 16 & 06:05:00 & 73 & 27 & 70 & 28 & 71 & 21 & 71 & 24 & 71 & 28 & 67 & 34 & 73 & 21 & 78 & 23 & 71 & 9 & 75 & 32 & 66 & 16 \\
\hline 17 & 06:05:20 & 75 & 35 & 73 & 21 & 74 & 23 & 71 & 18 & 68 & 27 & 66 & 12 & 71 & 29 & 77 & 22 & 72 & 16 & 72 & 15 & 67 & 15 \\
\hline 18 & 06:05:40 & 76 & 25 & 75 & 15 & 71 & 16 & 69 & 27 & 67 & 17 & 68 & 26 & 68 & 28 & 80 & 35 & 71 & 14 & 69 & 16 & 63 & 13 \\
\hline 19 & 06:06:00 & 74 & 31 & 75 & 26 & 68 & 19 & 70 & 24 & 68 & 21 & 68 & 24 & 69 & 13 & 79 & 30 & 71 & 17 & 70 & 14 & 64 & 11 \\
\hline 20 & 06:06:20 & 74 & 26 & 76 & 12 & 69 & 33 & 67 & 19 & 70 & 24 & 71 & 39 & 70 & 22 & 72 & 27 & 71 & 9 & 73 & 15 & 65 & 32 \\
\hline 21 & 06:06:40 & 73 & 25 & 78 & 18 & 68 & 23 & 67 & 34 & 72 & 35 & 75 & 33 & 72 & 22 & 71 & 18 & 68 & 15 & 72 & 15 & 62 & 17 \\
\hline 22 & 06:07:00 & 75 & 24 & 73 & 34 & 68 & 20 & 65 & 19 & 73 & 27 & 75 & 19 & 71 & 34 & 75 & 21 & 69 & 16 & 75 & 24 & 68 & 18 \\
\hline 23 & 06:07:20 & 77 & 11 & 72 & 27 & 70 & 26 & 68 & 36 & 73 & 19 & 76 & 17 & 69 & 20 & 75 & 18 & 71 & 10 & 75 & 24 & 70 & 18 \\
\hline 24 & 06:07:40 & 72 & 38 & 76 & 21 & 75 & 38 & 69 & 23 & 71 & 15 & 75 & 28 & 71 & 18 & 77 & 33 & 71 & 14 & 73 & 24 & 66 & 12 \\
\hline 25 & 06:08:00 & 72 & 43 & 76 & 31 & 72 & 37 & 71 & 19 & 71 & 19 & 71 & 38 & 69 & 18 & 80 & 27 & 68 & 21 & 74 & 16 & 67 & 16 \\
\hline 26 & 06:08:20 & 72 & 24 & 77 & 29 & 71 & 27 & 74 & 16 & 69 & 38 & 70 & 24 & 68 & 21 & 82 & 17 & 70 & 16 & 73 & 12 & 63 & 20 \\
\hline 27 & 06:08:40 & 71 & 24 & 70 & 35 & 74 & 16 & 73 & 25 & 69 & 24 & 71 & 27 & 68 & 26 & 82 & 35 & 69 & 13 & 74 & 13 & 67 & 16 \\
\hline 28 & 06:09:00 & 73 & 41 & 72 & 24 & 72 & 22 & 73 & 35 & 73 & 25 & 72 & 19 & 67 & 33 & 80 & 27 & 70 & 6 & 70 & 25 & 66 & 18 \\
\hline
\end{tabular}

Note: Speed (S) unit is mph; Volume (V) unit is number of vehicles, Det.x is the $\mathrm{x}$ number of detector. 
With the creation of this database, the data can be aggregated to different rolling steps for travel time prediction purpose. In this study, traffic detector data was aggregated to rolling steps of one to five minutes to investigate the effect of this aggregation on the analysis.

The data used in this study is for weekday only. The weekend data was excluded from the analysis because of the lower sample size of these days that may not be adequate for training and also because it is more challenging and useful to predict travel times for congested periods during weekdays. Due to the low traffic volumes in the nighttime period, traffic data for a time period starting from 6:00 AM to 9:00 PM is used in this study. This period is refer to as the daytime period in this study and is selected since it is more challenging to predict the travel time during the more congested traffic conditions encountered during this period compared to the other hours of the day.

Unfortunately, due to the malfunction of the newly installed detectors, a large proportion of the data were found to be missing in some days. The detailed information about the missing data is shown in Tables 3.4 and 3.5 for the training and test data, respectively.

The missing data percent varied from $0.2 \%$ to $94.6 \%$ for any given day. Further analysis was carried out to determine the proper threshold of percent of missing for the data to be used for modeling. This analysis will be discussed in the next chapter. 
Table 3.4: Missing Percent of Training Data

\begin{tabular}{|c|c|c|c|}
\hline ID & Day & Original missing observation & Original missing percent \\
\hline 1 & $06 / 13 / 2007$ & 15834 & $26.6 \%$ \\
\hline 2 & $06 / 14 / 2007$ & 15836 & $26.6 \%$ \\
\hline 3 & $06 / 15 / 2007$ & 10230 & $17.2 \%$ \\
\hline 4 & $06 / 18 / 2007$ & 176 & $0.2 \%$ \\
\hline 5 & $06 / 19 / 2007$ & 182 & $0.3 \%$ \\
\hline 6 & $06 / 20 / 2007$ & 174 & $0.2 \%$ \\
\hline 7 & $06 / 21 / 2007$ & 232 & $0.3 \%$ \\
\hline 8 & $06 / 22 / 2007$ & 186 & $0.3 \%$ \\
\hline 9 & $06 / 25 / 2007$ & 156 & $0.2 \%$ \\
\hline 10 & $06 / 26 / 2007$ & 356 & $0.5 \%$ \\
\hline 11 & $06 / 27 / 2007$ & 290 & $0.4 \%$ \\
\hline 12 & $06 / 28 / 2007$ & 344 & $0.5 \%$ \\
\hline 13 & $06 / 29 / 2007$ & 330 & $0.5 \%$ \\
\hline 14 & $07 / 02 / 2007$ & 1322 & $2.2 \%$ \\
\hline 15 & $07 / 03 / 2007$ & 600 & $1.0 \%$ \\
\hline 16 & $07 / 04 / 2007$ & 565 & $0.9 \%$ \\
\hline 17 & $07 / 05 / 2007$ & 398 & $0.6 \%$ \\
\hline 18 & $07 / 06 / 2007$ & 206 & $0.3 \%$ \\
\hline 19 & $07 / 09 / 2007$ & 206 & $0.3 \%$ \\
\hline 20 & $07 / 10 / 2007$ & 7982 & $13.4 \%$ \\
\hline 21 & $07 / 11 / 2007$ & 11058 & $18.6 \%$ \\
\hline 22 & $07 / 12 / 2007$ & 10876 & $18.3 \%$ \\
\hline 23 & $07 / 13 / 2007$ & 10980 & $18.4 \%$ \\
\hline 24 & $07 / 16 / 2007$ & 16484 & $27.7 \%$ \\
\hline 25 & $07 / 17 / 2007$ & 16218 & $27.3 \%$ \\
\hline 26 & $07 / 18 / 2007$ & 17330 & $29.1 \%$ \\
\hline 27 & $07 / 19 / 2007$ & 2086 & $3.5 \%$ \\
\hline 28 & $07 / 20 / 2007$ & 6290 & $10.5 \%$ \\
\hline 29 & $07 / 23 / 2007$ & 31336 & $52.7 \%$ \\
\hline 30 & $07 / 24 / 2007$ & 56199 & $94.6 \%$ \\
\hline 31 & $07 / 25 / 2007$ & 20466 & $34.4 \%$ \\
\hline 32 & $07 / 26 / 2007$ & 10874 & $18.3 \%$ \\
\hline 33 & $07 / 27 / 2007$ & 12816 & $21.5 \%$ \\
\hline 34 & $07 / 30 / 2007$ & 11018 & $18.5 \%$ \\
\hline 35 & $07 / 31 / 2007$ & 10896 & $18.3 \%$ \\
\hline 36 & $08 / 01 / 2007$ & 15836 & $26.6 \%$ \\
\hline 37 & $08 / 02 / 2007$ & 11390 & $4.3 \%$ \\
\hline 38 & $08 / 03 / 2007$ & 2570 & \\
\hline 0 & 155 & & \\
\hline
\end{tabular}

Note: Missing percent greater than $20 \%$ is marked with shaded grey color. 
Table 3.5: Missing Percent of Testing Data

\begin{tabular}{|c|c|c|c|}
\hline ID & Day & Original Missing Number & Original Missing Percent \\
\hline 1 & $08 / 06 / 2007$ & 288 & $0.4 \%$ \\
\hline 2 & $08 / 07 / 2007$ & 27830 & $46.8 \%$ \\
\hline 3 & $08 / 08 / 2007$ & 27830 & $46.8 \%$ \\
\hline 4 & $08 / 09 / 2007$ & 27820 & $46.8 \%$ \\
\hline 5 & $08 / 10 / 2007$ & 15699 & $26.4 \%$ \\
\hline 6 & $08 / 13 / 2007$ & 5866 & $9.8 \%$ \\
\hline 7 & $08 / 14 / 2007$ & 274 & $0.4 \%$ \\
\hline 8 & $08 / 15 / 2007$ & 1900 & $3.1 \%$ \\
\hline 9 & $08 / 16 / 2007$ & 174 & $0.2 \%$ \\
\hline 10 & $08 / 17 / 2007$ & 156 & $0.2 \%$ \\
\hline 11 & $08 / 20 / 2007$ & 3458 & $5.8 \%$ \\
\hline 12 & $08 / 22 / 2007$ & 16402 & $27.6 \%$ \\
\hline 13 & $08 / 23 / 2007$ & 4486 & $7.5 \%$ \\
\hline
\end{tabular}

Note: Missing percent greater than $20 \%$ is marked with shaded grey color.

\subsection{Data Imputation}

Data imputation is required for off-line travel time estimation, off-line travel time prediction model training and for on-line travel time prediction model implementation. A simple imputation method with interpolation strategy assessed by Van Lint (2004) was adopted in this study. This method satisfies both the estimation accuracy and computational efficiency requirements. Based on the different requirements of off-line and on-line applications, different methods will be used. Chapter 4 presents the results of simulation analysis conducted to validate this data imputation method. The following discussion presents the mathematical formulas of the interpolation methods.

Since the travel time estimation method is an off-line method, interpolation can be carried out in both the spatial and temporal directions (see Figure 3.3), given a route equipped with detectors $n \in\{1, \ldots, N\}$ located at specific locations on the corridor and a database of traffic measurements $M$ by these detectors at time period $t \in\{1, \ldots, T\}$. The location of each detector is denoted by $x_{n}$. 


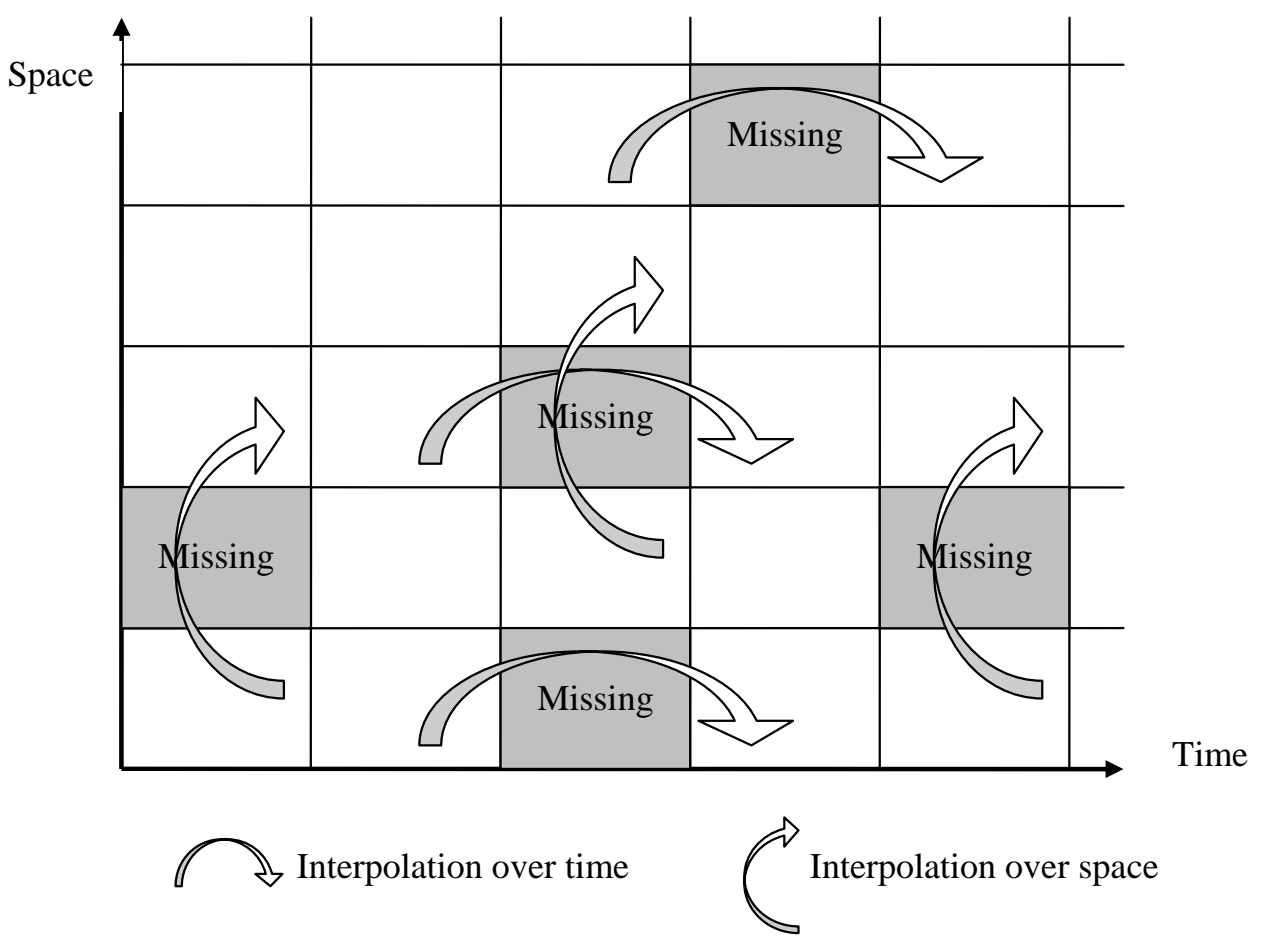

Figure 3.3: Simple Imputation Method: Interpolation over Space and Time

Suppose that for detector $n$ during time periods $t$ no data is available, the spatial interpolation procedure will be used to fill in this gap according to:

$$
M^{\text {space }}(n, t)=\left\{\begin{array}{cc}
M\left(n_{u}, t\right)+\frac{x_{n}-x_{u}}{x_{d}-x_{u}}\left(M\left(n_{d}, t\right)-M\left(n_{u}, t\right)\right) & 1<n<N \\
M\left(n_{d}, t\right) & n=1 \\
M\left(n_{u}, t\right) & n=N
\end{array}\right.
$$

where $M\left(n_{u}, t\right)$ and $M\left(n_{d}, t\right)$ are the first available upstream and downstream measurements for the detector location. $x_{u}$ and $x_{d}$ are the first available upstream and downstream detector locations. 
The temporal interpolation procedure fills in the gap according to:

$$
M^{\text {time }}(n, t)=\left\{\begin{array}{lc}
M\left(n, t_{p}\right)+\frac{t-t_{p}}{t_{n}-t_{p}}\left(M\left(n, t_{p}\right)-M\left(n, t_{n}\right)\right) & 1<t<T \\
M\left(n, t_{n}\right) & t=1 \\
M\left(n, t_{p}\right) & t=T
\end{array}\right.
$$

where $M\left(n, t_{p}\right)$ and $M\left(n, t_{n}\right)$ are the first available past and next measurement. $t_{p}$ and $t_{n}$ are the first available past and next time intervals.

These two interpolation methods were then combined to get the final value. The gap was filled with the minimum of both interpolates. The minimum is used because based on previous studies it was found that the travel times were usually underestimated because the traffic weaving effects is not properly considered. The minimum is used in this study as follows:

$$
M^{*}(n, t)=\min \left(M^{\text {space }}(n, t), M^{\text {time }}(n, t)\right)
$$

In the cases of structural detector failures, the temporal interpolation cannot be used because the data remains unavailable for long periods of time with these failures. In these cases, the missing values can only be estimated from spatial interpolation. An example data table of the training data interpolation is shown in Table 3-6.

For online applications, the time interpolation is not applicable because the future measurements are not available. Instead, an exponential moving average (EMA) method is proposed by Van Lint et al. (2004). This method can be combined with the spatial interpolation method mentioned above to get the final value. The method recognizes that traffic measurements from a given location exhibit strong autocorrelation over time. Missing or corrupt traffic measurements $M(t+1)$ from detector $n$ at time instant $t+1$ can 
be replaced by a forecast $f_{n}(t+1)$ of a simple time series model (in this case by an exponentially moving average) as follows:

$$
f_{n}(t+1)=f_{n}(t)+\alpha\left(M_{n}(t)-f_{n}(t)\right) \quad 0<\alpha<1
$$

This can also be expressed as:

$$
f_{n}(t+1)=\alpha M_{n}(t)+(1-\alpha) f_{n}(t) \quad 0<\alpha<1
$$

The discrete version of EMA is the following equation:

$$
f_{n}(t+1)=\alpha\left(M_{n}(t)+(1-\alpha) M_{n}(t-1)+(1-\alpha)^{2} M_{n}(t-2) \Lambda\right)+(1-\alpha)^{t} M_{n}(0)
$$

Typically $\alpha$ can be set to 0.4 which means that $40 \%$ is used as the weight of the measured value in the current step and $60 \%$ is used for the past measured values. In this study, ten period delays were used. This means that the method accounts for a fraction of $1-(1-\alpha)^{10} / \alpha=98.5 \%$ out of the total weight considered in the EMA.

In the above equations, $f_{n}(t)$ denotes the exponential forecast and $M_{n}(t)$ denotes the input value at time $t$ instant. Finally, the spatial and temporal interpolation methods are combined and each missing value is set to the minimum values obtained from the EMA and spatial interpolation method. An example data table of the testing data interpolation is shown in Table 3-7. 
Table 3.6: Training Data Interpolation Example

\begin{tabular}{|c|c|c|c|c|c|c|c|c|c|c|c|c|c|c|c|c|c|c|c|c|c|c|c|}
\hline \multirow{2}{*}{ ID } & \multirow{2}{*}{ timestamp } & \multicolumn{2}{|c|}{ Det.1 } & \multicolumn{2}{|c|}{ Det.2 } & \multicolumn{2}{|c|}{ Det.3 } & \multicolumn{2}{|c|}{ Det.4 } & \multicolumn{2}{|c|}{ Det.5 } & \multicolumn{2}{|c|}{ Det.6 } & \multicolumn{2}{|c|}{ Det.7 } & \multicolumn{2}{|c|}{ Det.8 } & \multicolumn{2}{|c|}{ Det.9 } & \multicolumn{2}{|c|}{ Det.10 } & \multicolumn{2}{|c|}{ Det.11 } \\
\hline & & S & $\mathbf{V}$ & $\mathrm{S}$ & $\mathbf{V}$ & $\mathrm{S}$ & $\mathbf{V}$ & $\mathrm{S}$ & $\mathbf{V}$ & S & $\mathbf{V}$ & S & $\mathbf{V}$ & $\mathrm{S}$ & $\mathbf{V}$ & $\mathrm{S}$ & $\mathbf{V}$ & $\mathrm{S}$ & $\mathbf{V}$ & S & $\mathbf{V}$ & S & $\mathbf{V}$ \\
\hline 1059 & 11:52:40 & & & 74 & 45 & 65 & 30 & 67 & 35 & 69 & 21 & & & 61 & 23 & 68 & 40 & 66 & 33 & & & & \\
\hline 1060 & 11:53:00 & 69 & 28 & 67 & 35 & 66 & 27 & 72 & 33 & 71 & 44 & 69 & 37 & 63 & 39 & 69 & 40 & 67 & 31 & 66 & 45 & 61 & 33 \\
\hline 1061 & 11:53:20 & & & & & & & & & & & & & & & & & & & & & & \\
\hline 1062 & $11: 53: 40$ & & & 63 & 28 & & & & & 71 & 33 & & & & & & & & & & & & \\
\hline 1063 & 11:54:00 & 69 & 29 & & & 65 & 34 & 71 & 27 & & & 71 & 36 & 60 & 24 & 72 & 45 & 67 & 29 & 73 & 28 & 68 & 27 \\
\hline
\end{tabular}

Space Filled

\begin{tabular}{|l|l|l|l|l|l|l|l|l|l|l|l|l|l|l|l|l|l|l|l|l|l|l|l|}
\hline 1059 & $11: 52: 40$ & 74 & 45 & 74 & 45 & 65 & 30 & 67 & 35 & 69 & 21 & 65 & 22 & 61 & 23 & 68 & 40 & 66 & 33 & 66 & 33 & 66 & 33 \\
\hline 1060 & $11: 53: 00$ & 69 & 28 & 67 & 35 & 66 & 27 & 72 & 33 & 71 & 44 & 69 & 37 & 63 & 39 & 69 & 40 & 67 & 31 & 66 & 45 & 61 & 33 \\
\hline 1061 & $11: 53: 20$ & & & & & & & & & & & & & & & & & & & & & & \\
\hline 1062 & $11: 53: 40$ & 63 & 28 & 63 & 28 & 65 & 29 & 68 & 31 & 71 & 33 & 71 & 33 & 71 & 33 & 71 & 33 & 71 & 33 & 71 & 33 & 71 & 33 \\
\hline 1063 & $11: 54: 00$ & 69 & 29 & 67 & 31 & 65 & 34 & 71 & 27 & 71 & 31 & 71 & 36 & 60 & 24 & 72 & 45 & 67 & 29 & 73 & 28 & 68 & 27 \\
\hline
\end{tabular}

Time Filled

\begin{tabular}{|l|l|l|l|l|l|l|l|l|l|l|l|l|l|l|l|l|l|l|l|l|l|l|l|}
\hline 1059 & $11: 52: 40$ & 71 & 26 & 74 & 45 & 65 & 30 & 67 & 35 & 69 & 21 & 69 & 49 & 61 & 23 & 68 & 40 & 66 & 33 & 68 & 37 & 63 & 29 \\
\hline 1060 & $11: 53: 00$ & 69 & 28 & 67 & 35 & 66 & 27 & 72 & 33 & 71 & 44 & 69 & 37 & 63 & 39 & 69 & 40 & 67 & 31 & 66 & 45 & 61 & 33 \\
\hline 1061 & $11: 53: 20$ & 69 & 28 & 65 & 32 & 66 & 29 & 72 & 31 & 71 & 39 & 69 & 37 & 62 & 34 & 70 & 41 & 67 & 31 & 68 & 40 & 63 & 31 \\
\hline 1062 & $11: 53: 40$ & 69 & 28 & 63 & 28 & 66 & 31 & 72 & 29 & 71 & 33 & 70 & 37 & 61 & 29 & 71 & 43 & 67 & 30 & 70 & 34 & 65 & 29 \\
\hline 1063 & $11: 54: 00$ & 69 & 29 & 64 & 32 & 65 & 34 & 71 & 27 & 70 & 33 & 71 & 36 & 60 & 24 & 72 & 45 & 67 & 29 & 73 & 28 & 68 & 27 \\
\hline
\end{tabular}

Training-Space\&Time Fill Combined

\begin{tabular}{|l|l|l|l|l|l|l|l|l|l|l|l|l|l|l|l|l|l|l|l|l|l|l|l|}
\hline 1059 & $11: 52: 40$ & 71 & 26 & 74 & 45 & 65 & 30 & 67 & 35 & 69 & 21 & 65 & 22 & 61 & 23 & 68 & 40 & 66 & 33 & 66 & 33 & 63 & 29 \\
\hline 1060 & $11: 53: 00$ & 69 & 28 & 67 & 35 & 66 & 27 & 72 & 33 & 71 & 44 & 69 & 37 & 63 & 39 & 69 & 40 & 67 & 31 & 66 & 45 & 61 & 33 \\
\hline 1061 & $11: 53: 20$ & 69 & 28 & 65 & 32 & 66 & 29 & 72 & 31 & 71 & 39 & 69 & 37 & 62 & 34 & 70 & 41 & 67 & 31 & 68 & 40 & 63 & 31 \\
\hline 1062 & $11: 53: 40$ & 63 & 28 & 63 & 28 & 65 & 29 & 68 & 29 & 71 & 33 & 70 & 33 & 61 & 29 & 71 & 33 & 67 & 30 & 70 & 33 & 65 & 29 \\
\hline 1063 & $11: 54: 00$ & 69 & 29 & 64 & 31 & 65 & 34 & 71 & 27 & 70 & 31 & 71 & 36 & 60 & 24 & 72 & 45 & 67 & 29 & 73 & 28 & 68 & 27 \\
\hline
\end{tabular}


Table 3.7: Testing Data Interpolation Example

\begin{tabular}{|c|c|c|c|c|c|c|c|c|c|c|c|c|c|c|c|c|c|c|c|c|c|c|c|}
\hline \multirow{2}{*}{ ID } & \multirow{2}{*}{ timestamp } & \multicolumn{2}{|c|}{ Det.1 } & \multicolumn{2}{|c|}{ Det.2 } & \multicolumn{2}{|c|}{ Det.3 } & \multicolumn{2}{|c|}{ Det.4 } & \multicolumn{2}{|c|}{ Det.5 } & \multicolumn{2}{|c|}{ Det.6 } & \multicolumn{2}{|c|}{ Det.7 } & \multicolumn{2}{|c|}{ Det.8 } & \multicolumn{2}{|c|}{ Det.9 } & \multicolumn{2}{|c|}{ Det.10 } & \multicolumn{2}{|c|}{ Det.11 } \\
\hline & & $S$ & $\mathbf{V}$ & $S$ & $\mathbf{V}$ & $\mathbf{S}$ & $\mathbf{V}$ & $S$ & $\mathbf{V}$ & $S$ & $\mathbf{V}$ & $S$ & $\mathbf{V}$ & $\mathrm{S}$ & $\mathbf{V}$ & $\mathrm{S}$ & $\mathbf{V}$ & $\mathrm{S}$ & $\mathbf{V}$ & $\mathrm{S}$ & V & $\mathrm{S}$ & V \\
\hline 709 & 09:56:00 & 71 & 46 & 72 & 22 & 61 & 28 & & & 65 & 19 & 67 & 26 & 59 & 48 & 70 & 37 & 60 & 30 & & & 66 & 31 \\
\hline 710 & 09:56:20 & 68 & 44 & 70 & 31 & 69 & 30 & 70 & 37 & 61 & 21 & 71 & 38 & 62 & 28 & 70 & 39 & 64 & 30 & 66 & 43 & 67 & 25 \\
\hline 711 & 09:56:40 & 68 & 27 & & & & & 71 & 22 & & & & & & & 69 & 31 & 59 & 27 & 61 & 34 & 72 & 29 \\
\hline 712 & 09:57:00 & & & & & & & & & & & & & & & & & & & & & & \\
\hline 713 & 09:57:20 & 68 & 29 & 70 & 30 & 64 & 32 & 69 & 31 & 69 & 34 & 67 & 43 & 66 & 27 & 73 & 38 & 68 & 26 & 65 & 49 & 67 & 34 \\
\hline
\end{tabular}

Space Filled

\begin{tabular}{|l|l|l|l|l|l|l|l|l|l|l|l|l|l|l|l|l|l|l|l|l|l|l|l|}
\hline 709 & $09: 56: 00$ & 71 & 46 & 72 & 22 & 61 & 28 & 63 & 24 & 65 & 19 & 67 & 26 & 59 & 48 & 70 & 37 & 60 & 30 & 63 & 30 & 66 & 31 \\
\hline 710 & $09: 56: 20$ & 68 & 44 & 70 & 31 & 69 & 30 & 70 & 37 & 61 & 21 & 71 & 38 & 62 & 28 & 70 & 39 & 64 & 30 & 66 & 43 & 67 & 25 \\
\hline 711 & $09: 56: 40$ & 68 & 27 & 69 & 26 & 70 & 24 & 71 & 22 & 71 & 24 & 71 & 26 & 70 & 28 & 69 & 31 & 59 & 27 & 61 & 34 & 72 & 29 \\
\hline 712 & $09: 57: 00$ & & & & & & & & & & & & & & & & & & & & \\
\hline 713 & $09: 57: 20$ & 68 & 29 & 70 & 30 & 64 & 32 & 69 & 31 & 69 & 34 & 67 & 43 & 66 & 27 & 73 & 38 & 68 & 26 & 65 & 49 & 67 & 34 \\
\hline
\end{tabular}

EMA Filled

\begin{tabular}{|l|l|l|l|l|l|l|l|l|l|l|l|l|l|l|l|l|l|l|l|l|l|l|l|}
\hline 709 & $09: 56: 00$ & 71 & 46 & 72 & 22 & 61 & 28 & 67 & 38 & 65 & 19 & 67 & 26 & 59 & 48 & 70 & 37 & 60 & 30 & 71 & 32 & 66 & 31 \\
\hline 710 & $09: 56: 20$ & 68 & 44 & 70 & 31 & 69 & 30 & 70 & 37 & 61 & 21 & 71 & 38 & 62 & 28 & 70 & 39 & 64 & 30 & 66 & 43 & 67 & 25 \\
\hline 711 & $09: 56: 40$ & 68 & 27 & 71 & 30 & 65 & 31 & 71 & 22 & 64 & 25 & 69 & 36 & 62 & 33 & 69 & 31 & 59 & 27 & 61 & 34 & 72 & 29 \\
\hline 712 & $09: 57: 00$ & 69 & 35 & 71 & 30 & 65 & 31 & 69 & 31 & 64 & 25 & 69 & 36 & 62 & 33 & 69 & 35 & 61 & 27 & 65 & 35 & 68 & 28 \\
\hline 713 & $09: 57: 20$ & 68 & 29 & 70 & 30 & 64 & 32 & 69 & 31 & 69 & 34 & 67 & 43 & 66 & 27 & 73 & 38 & 68 & 26 & 65 & 49 & 67 & 34 \\
\hline
\end{tabular}

\section{Space \& EMA Fill Combined}

\begin{tabular}{|l|l|l|l|l|l|l|l|l|l|l|l|l|l|l|l|l|l|l|l|l|l|l|l|}
\hline 709 & $09: 56: 00$ & 71 & 46 & 72 & 22 & 61 & 28 & 63 & 24 & 65 & 19 & 67 & 26 & 59 & 48 & 70 & 37 & 60 & 30 & 63 & 30 & 66 & 31 \\
\hline 710 & $09: 56: 20$ & 68 & 44 & 70 & 31 & 69 & 30 & 70 & 37 & 61 & 21 & 71 & 38 & 62 & 28 & 70 & 39 & 64 & 30 & 66 & 43 & 67 & 25 \\
\hline 711 & $09: 56: 40$ & 68 & 27 & 69 & 26 & 65 & 24 & 71 & 22 & 64 & 24 & 69 & 26 & 62 & 28 & 69 & 31 & 59 & 27 & 61 & 34 & 72 & 29 \\
\hline 712 & $09: 57: 00$ & 69 & 35 & 71 & 30 & 65 & 31 & 69 & 31 & 64 & 25 & 69 & 36 & 62 & 33 & 69 & 35 & 61 & 27 & 65 & 35 & 68 & 28 \\
\hline 713 & $09: 57: 20$ & 68 & 29 & 70 & 30 & 64 & 32 & 69 & 31 & 69 & 34 & 67 & 43 & 66 & 27 & 73 & 38 & 68 & 26 & 65 & 49 & 67 & 34 \\
\hline
\end{tabular}

Note: Speed (S) unit is mph; Volume (V) unit is number of vehicles. 
Van Lint et al. (2003) tested this simple imputation method using simulation and showed that the simple method outperform the more complicated methods such as the first order Lighthill, Witham and Richards (LWR) model used by Haj-Salem and Lebacque (2002).

\subsection{Speed Transformation}

The Smart SunGuide system records the spot time mean speed in a 20 seconds interval. The space mean speed $\left(\mu_{S}\right)$ is always equal or smaller than the time mean speed ( $\mu_{T}$ ) with the difference being proportional to the speed variance $\left(\sigma_{S}^{2}, \sigma_{T}^{2}\right)$. The explanation is that low speed vehicles influence the average of space mean speed more than vehicles with high speed, while both low speed and high speed vehicles have the same influence on the average time mean speed.

Wardrop (1952) derived the relationship between the space mean speed and time mean speed as follows:

$$
\bar{\mu}_{T}=\bar{\mu}_{S}+\frac{\sigma_{S}^{2}}{\bar{\mu}_{S}}
$$

This equation is applied to estimate the time mean speed from the space mean speed. However, in our case, the time mean speed is available, and the space-mean speed needs to be estimated from time-mean speed. Rakha and Zhang (2005) proposed a formulation for estimating space mean speeds from time-mean speeds with a small margin of error (within 0 to 1 percent) as follows:

$$
\bar{\mu}_{S}=\bar{\mu}_{T}-\frac{\sigma_{T}^{2}}{\bar{\mu}_{T}}
$$


They also derived the relationship between the time mean speed and space mean speed variance:

$$
\sigma_{S}^{2}=\sigma_{T}^{2}-\left(\frac{\sigma_{T}^{2}}{\bar{\mu}_{T}}\right)^{2}
$$

Since the space mean speed and its variance is not recorded, the only possible way is to estimate space-mean speed is based on the time mean speed and its estimated variance using the combination of Equations (3-8) and (3-9).

However, the model parameters based on field data are expected to be specific to the local roadway and traffic stream characteristics. Based on field observed data, Van Lint (2004) found that in congested conditions, the variance of speed is nearly constant, while in free-flow conditions, the variance is a steeply increasing function of mean speed. He presented a linear model based on field data as follow:

$$
\hat{\sigma}_{S}=\left\{\begin{array}{cc}
0.5 \mu_{T}-34 & \mu_{T} \geq 74 \\
0.02 \mu_{T}+5 & \text { otherwise }
\end{array} \quad \text { unit }: \mathrm{km} / \mathrm{h}\right.
$$

Combining this equation with Equations (3-8) and (3-9), the space mean speed can be estimated based on time mean speed.

Garber and Hoel (2002) derived a linear regression model between time mean and space mean speed based on field data collected from Virginia limited access highways, as follows:

$$
\bar{\mu}_{T}=0.966 \bar{\mu}_{S}+3.541 \quad \text { unit }: \mathrm{km} / \mathrm{h}
$$

This empirical linear equation is very straight forward and easy for application. However, for application it needs to be calibrated and customized to the local condition. In this study, linear regression model was used to model the relationship between the 
time mean speed and space mean speed for different speed conditions. The data was obtained using the micro-simulation software of AIMSUN with a short freeway segment coded in the model. Different traffic conditions were modeled with varied input traffic volumes, time mean speed, and standard deviation of time mean speed. The space mean speed for various inputs was collected from the output of AIMSUN. The output data is plotted in Figure 3.4.

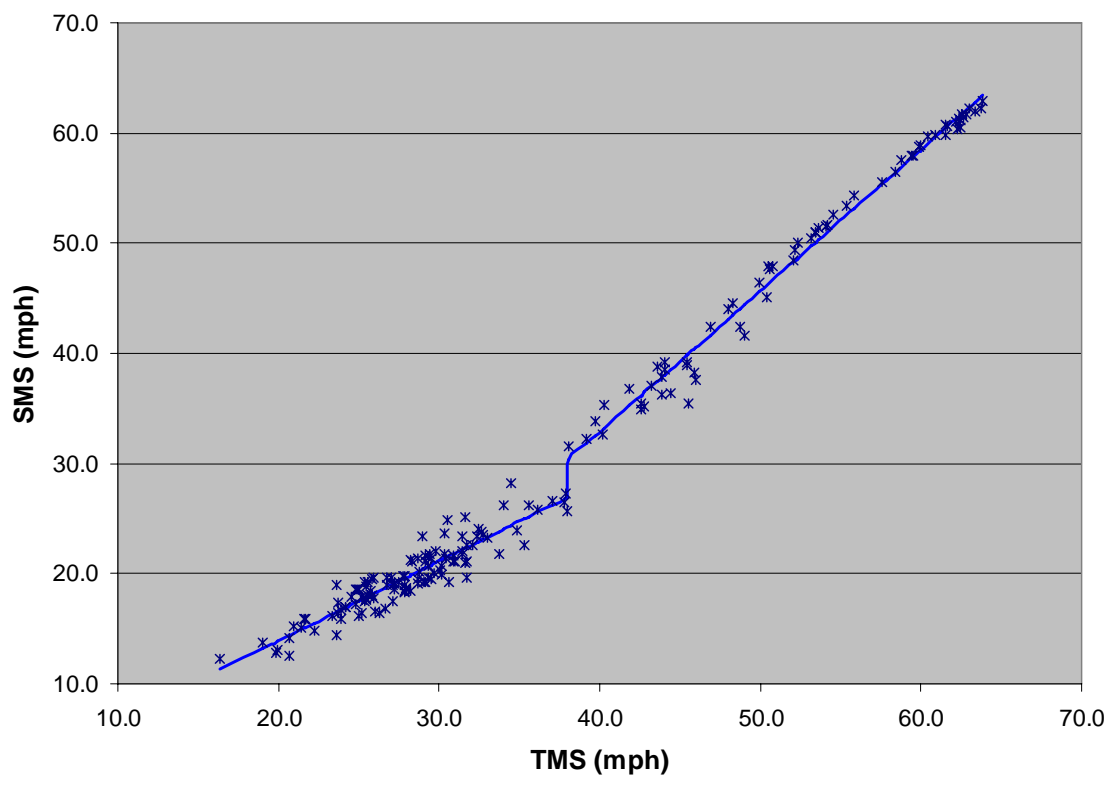

Figure 3.4: TMS vs SMS Plot

As discussed above, Garber and Hoel (2002) fitted the relationship with one straight line. Van Lint (2004), on the other hand, found that the speed relationship was different for high speed condition compared to low speed condition. The results in Figure 3.4 apparently show two clusters: one represents the un-congested conditions and the other represents the congested conditions. As a result, two separate linear regression 
models were developed to fit the data. To develop the two linear models, the key point was to find the transition point of the two clusters. When deriving the regression equations using the Excel software, the transition point was varied and the sum of residuals of the two straight lines was calculated and plotted in Figure 3.5. The equations with the lowest total residual were selected to represent the model with the transition point found to be 38 miles/hour.

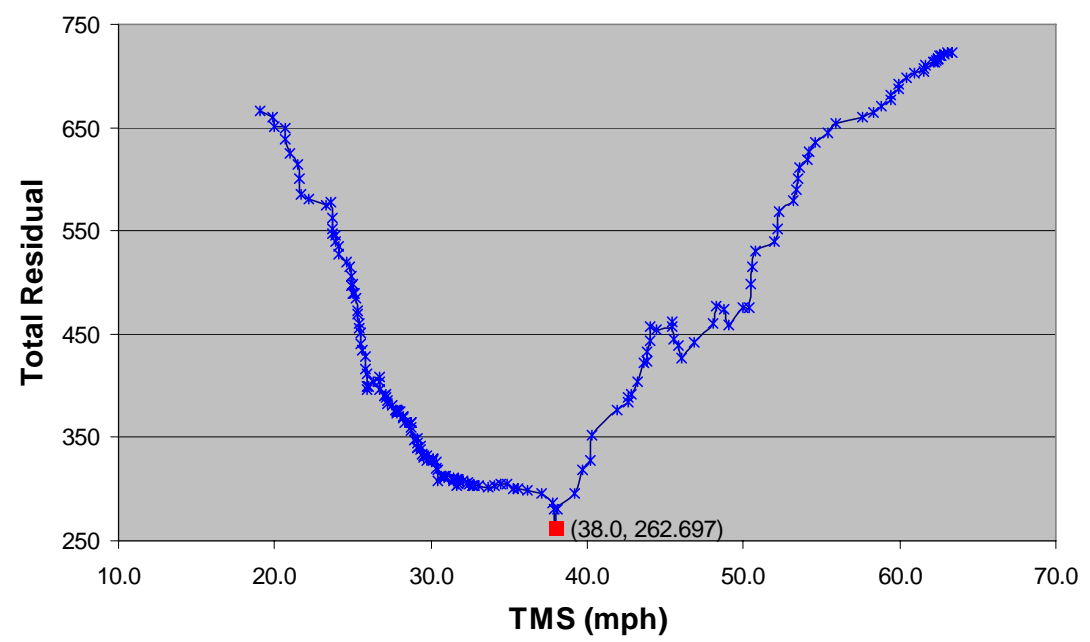

Figure 3.5: Total Residual with Varied Transition point of Regression Models

For speed higher than $65 \mathrm{mph}$, the time mean speed was found to approach the space mean speed, as shown in Figure 3.5. Van Lint (2004) also found that the space mean speed becomes close to the time mean speed when the speed is high. Thus, the time mean speed was set to equal the space mean speed for speeds higher than $65 \mathrm{mph}$ in this study. The final speed transformation formula is a combination of the three linear lines as shown in Equation 3-12. 


$$
\hat{\mu}_{S}=\left\{\begin{array}{lll}
0.72 \mu_{T}-0.59 & \mu_{T} \leq 38 & R^{2}=0.86 \\
1.28 \mu_{T}-18.39 & 38<\mu_{T} \leq 65 & R^{2}=0.99 \\
\mu_{T} & \mu_{T}>65 &
\end{array} \quad \text { unit: } \mathrm{mph}\right.
$$

As shown in Equation 3-12, for low speed condition, $R^{2}$ is 0.86 , which is lower than the $R^{2}$ of the median speed condition of 0.99 . However, this value is still high enough and is an acceptable value. Further validation of this transformation equation will be carried out in Chapter 4 . 


\section{CHAPTER 4}

FREEWAY TRAVEL TIME ESTIMATION

\subsection{Introduction}

Travel time can be directly measured with probe vehicle techniques. But they are more expensive and not available in FDOT for this research. Since most of the Florida freeways are instrumented with point traffic detectors. The traffic detector data can be used to estimate the travel times for further prediction modeling. After the data cleaning and speed transformation, this chapter will investigate a travel time estimation method called trajectory algorithm to calculate the link travel time based on the already preprocessed data.

The class of trajectory algorithm assumes an imaginary vehicle traversing through a database of measured mean speeds on detector locations along the route. The PLSB method studied by Van Lint and Van der Zijpp (2003) leads to more accurate travel time estimates compared to constant speed trajectory methods, as discussed in Chapter 2 . The PLSB method assumes speed as a convex combination of upstream and downstream speeds. The speed is assumed to vary linearly with the distance between the upstream and downstream detectors, as shown in Figure 4.1. The obtained section-level travel times are added up as needed to constitute the path-level travel time. A modification to the method is proposed in this study to assume that the speed is linearly changing with time rather than with distance, as assumed in the Van Lint study. This implies that the drivers apply a constant acceleration or deceleration rate when a speed change is needed on the segment between two detectors. For this reason, the new method used in this 
study is the Piecewise Constant Acceleration Based method (PCAB). Both the PLSB, PCAB methods and other constant speed methods found in literatures were tried in this study to calculate the estimated travel time. The results were compared based on simulation data collected from the AIMSUN software to determine which method has better performance and will be used for travel time estimation.

Figure 4.1 shows a map of the trajectory of a vehicle traversing through a route. To calculate the travel time for a route, first we developed a formula to calculate the exit point $\left(x^{*}, t^{*}\right)$ of a space-time region $(n, p)$ given an entry point $\left(x^{0}, t^{0}\right)$. Below are the associated mathematical formulas for the PLSB and PCAB method.

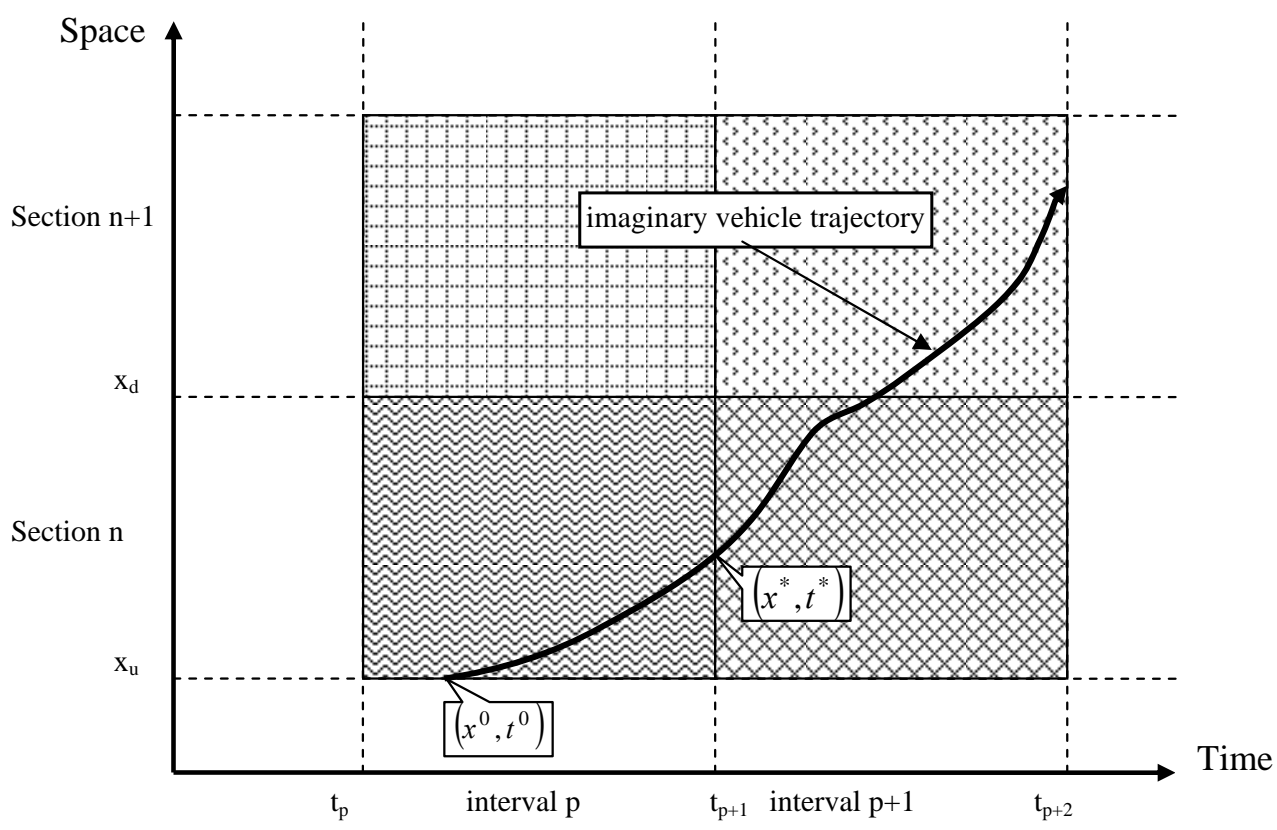

Figure 4.1: Vehicle Trajectory Demonstration of Linear Speed Method 


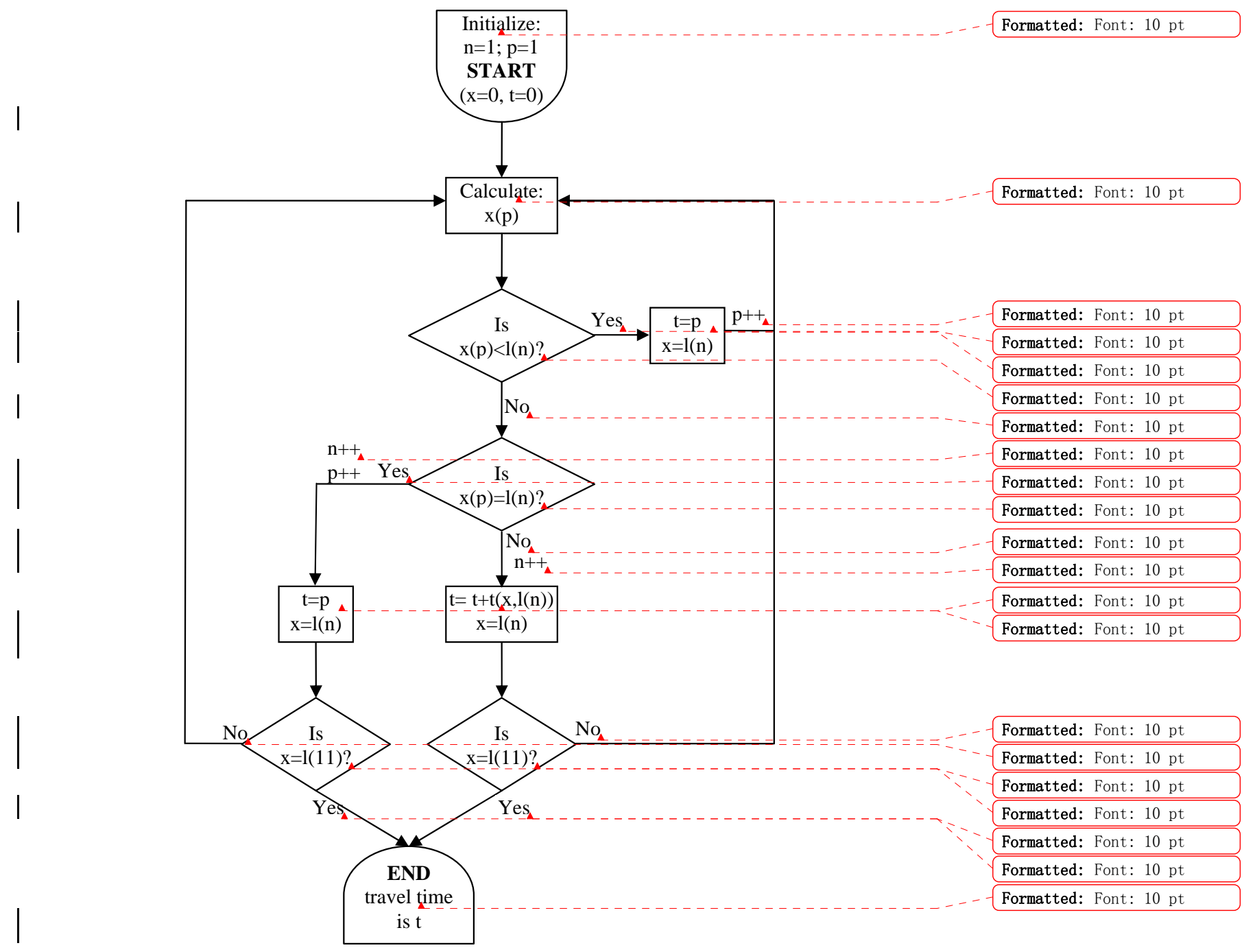

Note: $\mathrm{n}$ is the number of detector; $\mathrm{p}$ is time period; $\mathrm{x}(\mathrm{t})$ is location at time $\mathrm{t}$; $\mathrm{l}(\mathrm{n})$ is the location of detector

Figure 4.2: Flow Chart of the Route Travel Time Estimation Method

The estimated travel time calculation process flow chart that is applied for both the PLSB and PCAB methods are shown in Figure 4.2. These methods were applied in a program coded in $\mathrm{C}++$ with connections to the SQL database tables. This allows the estimation of travel time for different rolling steps. 
For the PLSB method the space mean speed is assumed to be a linear function of distance, as follows:

$$
v(x)=\mu_{u}(n, p)+\frac{x-x_{u}}{x_{d}-x_{u}}\left(\mu_{d}(n, p)-\mu_{u}(n, p)\right)
$$

Then, the time location function can be derived from the following equation:

$$
\begin{aligned}
& t(x)=t^{o}+\int_{x^{o}}^{x} \frac{d x}{v(x)}=t^{o}+\int_{x^{0}}^{x} \frac{d x}{\mu_{u}(n, p)+\frac{x-x_{u}}{x_{d}-x_{u}}\left(\mu_{d}(n, p)-\mu_{u}(n, p)\right)} \\
& =t^{o}+\frac{x_{d}-x_{u}}{\mu_{d}(n, p)}\left(\ln \left(\mu_{u}(n, p)+\frac{x-x_{u}}{x_{d}-x_{u}}\left(\mu_{d}(n, p)-\mu_{u}(n, p)\right)\right)-\ln \left(\mu_{u}(n, p)+\frac{x^{0}-x_{u}}{x_{d}-x_{u}}\left(\mu_{d}(n, p)-\mu_{u}(n, p)\right)\right)\right)
\end{aligned}
$$

and,

$$
x(t)=x_{u}-\frac{\mu_{u}\left(x_{d}-x_{u}\right)}{\mu_{d}-\mu_{u}}+\left(\left(x^{0}-x_{u}\right)+\frac{\mu_{d}-\mu_{u}}{\mu_{d}}\left(x_{d}-x_{u}\right)\right) e^{\frac{\mu_{d}-\mu_{u}}{x_{d}-x_{u}}\left(t-t^{0}\right)}
$$

There are two scenarios: one is the vehicle reached the next detector location during the time period; the other one is at the end of the time period the vehicle is still not reached the next detector location. Then the exit points for both scenarios can be calculated through the following equation:

$$
\left(x^{*}, t^{*}\right)= \begin{cases}\left(x_{d}, t\left(x_{d}\right)\right) & t\left(x_{d}\right)<t_{p+1} \\ \left(x\left(t_{p+1}\right), t_{p+1}\right) & x\left(t_{p+1}\right)<x_{d}\end{cases}
$$

By applying this method sequentially, one can get the whole link travel time.

For the PCAB method the space mean speed is assumed to be a linear function of time. In another word, the acceleration rate is constant and can be obtained using the following equation. 


$$
a=\frac{\mu_{d}^{2}(n, p)-\mu_{u}^{2}(n, p)}{2\left(x_{d}-x_{u}\right)}
$$

The speed function is:

$$
v(t)=\mu_{u}(n, p)+a t=\mu_{u}(n, p)+\frac{\mu_{d}^{2}(n, p)-\mu_{u}^{2}(n, p)}{2\left(x_{d}-x_{u}\right)}\left(t-t^{0}\right)
$$

Then location time function is derived through the following equation:

$$
x(t)=x_{u}+\int_{t^{0}}^{t} v(t) d t=x_{u}+\mu_{u}(n, p)\left(t-t^{0}\right)+\frac{\mu_{d}^{2}(n, p)-\mu_{u}^{2}(n, p)}{4\left(x_{d}-x_{u}\right)}\left(t-t^{0}\right)^{2}
$$

where $\mu_{u}(n, p)$ is the upstream detector speed, $\mu_{d}(n, p)$ is the downstream detector speed, $x_{u}$ is the upstream detector location, $x_{d}$ is the downstream detector location.

\subsection{Validation of Travel Time Estimation Method with Simulation Data}

Traffic micro-simulation tools have the capability to model individual vehicle's behavior through microscopic traffic models such as car-following models, lane-changing models and gap-accepting models. One of the widely used tools, AIMSUN developed by Transportation Simulation Systems (TSS), was used in this study as the simulation tool to test the data preprocessing and the travel time estimation methods proposed in this study. The freeway corridor of I-95 (8.25 miles) with detectors installed along the freeway segment was simulated in AIMSUN. Different scenarios with different traffic demands and incident conditions were generated using the model. Traffic detector data such as time mean speeds and volumes were collected from the simulation model. The real travel time assessed by AIMSUN for the simulated highway segment was also recorded. With this data, the performance of the PLSB method, PCAB method and other constant speed methods were tested and compared. The simulation data was also used to test the data 
imputation method and speed transformation formula, discussed earlier in this dissertation. To test the data imputation method, missing simulated detector data were created randomly with a program to emulate the real detector malfunctions.

To test the travel time estimation method, different traffic conditions including varied congestion levels and incident conditions were generated in the simulation model. Varied congestion conditions were achieved by changing the input volumes in the simulation. A total 15 hour period was modeled in AIMSUN from 6:00 AM to 9:00 PM. The modeled traffic conditions include a morning peak, an afternoon peak, and a PM peak. In addition, a two lane blockage incident was also introduced in the simulation during the morning peak to test the used model performance under incident conditions.

Table 4.1: Traffic Conditions of the Simulated Network

\begin{tabular}{|c|c|c|c|c|c|}
\hline $\begin{array}{c}\text { Time } \\
\text { (hour) }\end{array}$ & $\begin{array}{c}\text { Car } \\
\text { Volume }\end{array}$ & $\begin{array}{c}\text { Truck } \\
\text { Volume }\end{array}$ & $\begin{array}{c}\text { Total } \\
\text { Volume }\end{array}$ & $\begin{array}{c}\text { V/C } \\
\text { Ratio }\end{array}$ & Notes \\
\hline 1 & 6349 & 334 & 7017 & 0.64 & \\
\hline 2 & 7618 & 401 & 8420 & 0.77 & \\
\hline 3 & 8888 & 468 & 9824 & 0.89 & \\
\hline 4 & 9523 & 501 & 10525 & 0.96 & \\
\hline 5 & 9523 & 501 & 10525 & 0.96 & $\begin{array}{c}10: 00-10: 30 \text { two } \\
\text { center lanes blocked }\end{array}$ \\
\hline 6 & 8888 & 468 & 9824 & 0.89 & \\
\hline 7 & 7618 & 401 & 8420 & 0.77 & \\
\hline 8 & 6983 & 368 & 7718 & 0.70 & \\
\hline 9 & 7618 & 401 & 8420 & 0.77 & \\
\hline 10 & 8888 & 468 & 9824 & 0.89 & \\
\hline 11 & 9523 & 501 & 10525 & 0.96 & \\
\hline 12 & 10158 & 535 & 11227 & 1.02 & \\
\hline 13 & 8253 & 434 & 9122 & 0.83 & \\
\hline 14 & 6983 & 368 & 7718 & 0.70 & \\
\hline 15 & 6349 & 334 & 7017 & 0.64 & \\
\hline
\end{tabular}

Note 1: Volume unit is vehicles per hour;

Note 2: For the period with incident condition, the reported V/C ratio is for incident-free situation.

To assess the congestion level in the system, an approximate $\mathrm{V} / \mathrm{C}$ ratio was calculated based on the demand assuming a capacity of 2,200 vphpl and taking into 
account the percent of truck vehicle of $5 \%$ with the passenger car equivalent parameter for the truck assumed to be 2. The approximate V/C ratio of the modeled corridor for different hours of the day is listed in Table 4.1. As shown in Table 4-1, different traffic congestion conditions were included in the evaluation.

The performance measures used in assessing the performance of travel time estimation were the mean absolute error (MAE) and mean absolute percentage error (MAPE), calculated as follows:

$$
\begin{aligned}
& M A E=\frac{1}{n} \sum_{i}\left|O_{i}-D_{i}\right| \\
& M A P E=\frac{1}{n} \sum_{i} \frac{\left|O_{i}-D_{i}\right|}{D_{i}}
\end{aligned}
$$

where $O_{i}$ is the model output value and $D_{i}$ is the desired value.

With the PLSB method, PCAB method and other constant speed methods, travel time is estimated and compared to the real travel time as assessed by AIMSUN for the highway section. Table 4.2 lists the performance measurements for the five different rolling steps assessed in this study. Figure 4.3 shows the PCAB estimated travel time compared to the real link travel time. In addition, results from the constant speed-based methods including the half distance speed method, the minimum speed method and average speed method are also included in Table 4.2 for comparison purpose. The results show that the PLSB, average speed and the PCAB method are better than other constant speed methods. The average speed method which can be classified as a constant speed method was found to work as well as the PCAB method. 


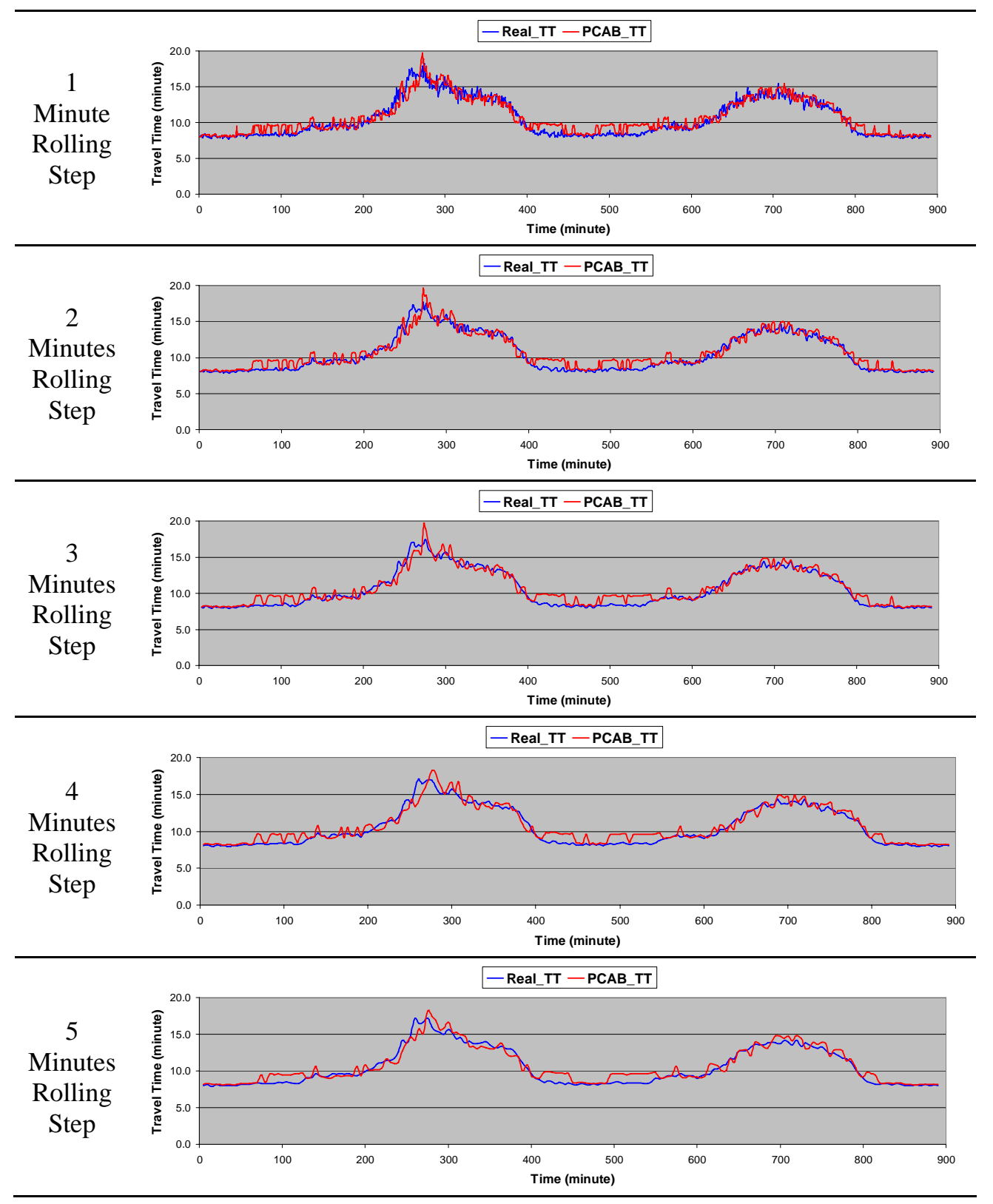

Figure 4.3: PCAB Estimated Travel Time Compared to Real Travel Time 
Table 4.2: Performance Measure of the PCAB Method Comparing to Other Methods

\begin{tabular}{|c|c|c|c|c|c|c|}
\hline \multirow{2}{*}{ Rolling Step } & Estimation Method & $\begin{array}{c}\text { Half } \\
\text { Distance }\end{array}$ & $\begin{array}{c}\text { Minimum } \\
\text { Speed }\end{array}$ & PLSB & $\begin{array}{c}\text { Average } \\
\text { Speed }\end{array}$ & PCAB \\
\hline \multirow{2}{*}{$\begin{array}{c}\text { One } \\
\text { minute }\end{array}$} & MAE (minutes) & 0.94 & 2.89 & 0.45 & 0.27 & 0.26 \\
\cline { 2 - 7 } & MAPE & $8.9 \%$ & $27.6 \%$ & $4.3 \%$ & $2.6 \%$ & $2.5 \%$ \\
\hline \multirow{2}{*}{$\begin{array}{c}\text { Two } \\
\text { minutes }\end{array}$} & MAE (minutes) & 0.97 & 2.89 & 0.47 & 0.29 & 0.29 \\
\cline { 2 - 7 } $\begin{array}{c}\text { Three } \\
\text { minutes }\end{array}$ & MAPE & $9.2 \%$ & $27.5 \%$ & $4.5 \%$ & $2.7 \%$ & $2.7 \%$ \\
\cline { 2 - 7 } $\begin{array}{c}\text { Four } \\
\text { minutes }\end{array}$ & MAE (minutes) & 0.97 & 2.88 & 0.45 & 0.30 & 0.29 \\
\hline \multirow{2}{*}{$\begin{array}{c}\text { Five } \\
\text { minutes }\end{array}$} & MAPE & $9.2 \%$ & $27.4 \%$ & $4.3 \%$ & $2.8 \%$ & $2.8 \%$ \\
\hline \multirow{2}{*}{\begin{tabular}{c} 
Average \\
\cline { 2 - 7 }
\end{tabular}} & MAE (minutes) & 0.98 & 2.90 & 0.46 & 0.27 & 0.29 \\
\cline { 2 - 7 } & MAPE & $9.4 \%$ & $27.6 \%$ & $4.4 \%$ & $2.7 \%$ & $2.8 \%$ \\
\hline \multirow{2}{*}{} & MAE (minutes) & 9.01 & 2.86 & 0.44 & 0.26 & 0.26 \\
\cline { 2 - 7 } & MAPE & 9.974 & $27.3 \%$ & $4.1 \%$ & $2.5 \%$ & $2.5 \%$ \\
\hline
\end{tabular}

Figure 4.4 shows the speed pattern and the vehicle trajectory of the average speed and PCAB method assuming the speed measurements will not change for a long period so that the vehicle can travel through the segment. It can be seen that the segment travel times calculated using the average speed and PCAB method are the same.

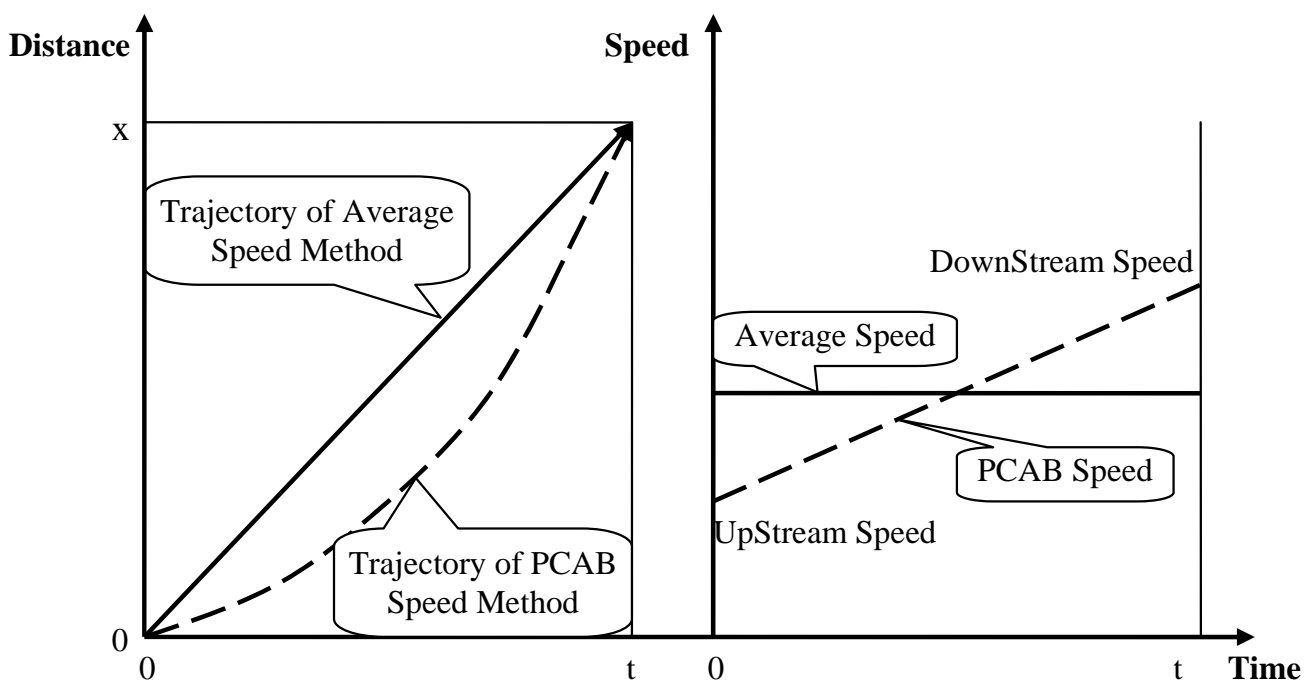

Figure 4.4: Experiment of Average Speed Method and PCAB Speed Method 
In this study, the PCAB method was selected as the final method to calculate the estimated travel time. From Table 4.2 and Figure 4.3, it can be seen that the PCAB travel time estimation method performs well for all traffic conditions. Larger differences were found between estimated and assessed travel time during incident condition, which may be expected due the difficulty in relating the speed at the upstream and downstream locations of the incident location to the traffic conditions on the segment because of the complex traffic behavior under incident situation. From Figure 4.3, it appears that the travel time estimates appear to frequently oscillate for a rolling step of one minute. With the rolling step increased to two minutes, the estimated travel time become much more stable. This may give us an indication of the appropriate rolling step, which will be further discussed in Chapter 5.

\subsection{Validation of Data Preprocessing Method with Simulation Data}

In this study, the traffic detector data was collected every 20 seconds, which is the same interval used to collect the data from the field detectors along the I-95 corridor. This simulated data were used in this study to test the data preprocessing method including the data imputation methods and data transformation formula.

\subsubsection{Validation of Data Imputation Method}

Before aggregated to different rolling steps, a program was written to randomly create missing data. Two types of missing data were created: random missing data and structure missing data which emulate the malfunction of some detectors for some time periods. Table 4.3 lists the results of the data imputation performance under various situations. 
Table 4.3: Influence of Missing Data

\begin{tabular}{|c|c|c|c|c|c|c|c|c|}
\hline \multirow{2}{*}{ Rolling Step } & \multirow{3}{*}{ Measurement } & \multicolumn{4}{|c|}{ Missing Data Percent } & \multicolumn{3}{c|}{$\begin{array}{c}\text { Number of } \\
\text { Malfunction Detector }\end{array}$} \\
\cline { 3 - 9 } & & $10 \%$ & $20 \%$ & $30 \%$ & $40 \%$ & $50 \%$ & 2 & 4 \\
\hline \multirow{2}{*}{ One minute } & MAE (minutes) & 0.440 & 0.597 & 0.774 & 0.894 & 1.071 & 0.099 & 2.067 \\
\cline { 2 - 9 } & MAPE & $4.2 \%$ & $5.7 \%$ & $7.4 \%$ & $8.5 \%$ & $10.2 \%$ & $0.9 \%$ & $19.7 \%$ \\
\hline
\end{tabular}

From Table 4.3, one can conclude that random missing data affect the estimation accuracy less than the missing data due structure malfunctions, if the number of malfunctioned detectors is four. In the case of only two malfunctioned detectors, the impact on the detection accuracy is small. When the missing percent increased from $10 \%$ to $50 \%$, the MAPE of the estimated travel time increased from $4.2 \%$ to $10.2 \%$ compared to the assessed travel time. Accurate data is needed since the estimated travel time will be used in the travel time prediction process, which will introduce further error in the process. In this study, a threshold of the percent of missing data in a given day was set to $20 \%$ with the corresponding MAPE of 5.7\%. In future studies, the impact of different errors in the estimation of travel time on the prediction accuracy should be investigated. With the criteria only those days with missing data less than 20\% were selected for the prediction model development. For the influence of the structure malfunction of detectors, it was found that two detector malfunctions do not affect the estimation accuracy. However, when the number of malfunction detectors increase to 4, the MAPE approached 20\%, which is not acceptable. It appears that when malfunctioned detectors exceed two detectors on the investigated segment, the estimated travel time is not reliable enough. Figure 4.5 and Figure 4.6 show the plot of the estimated travel time comparing to the real travel time. 


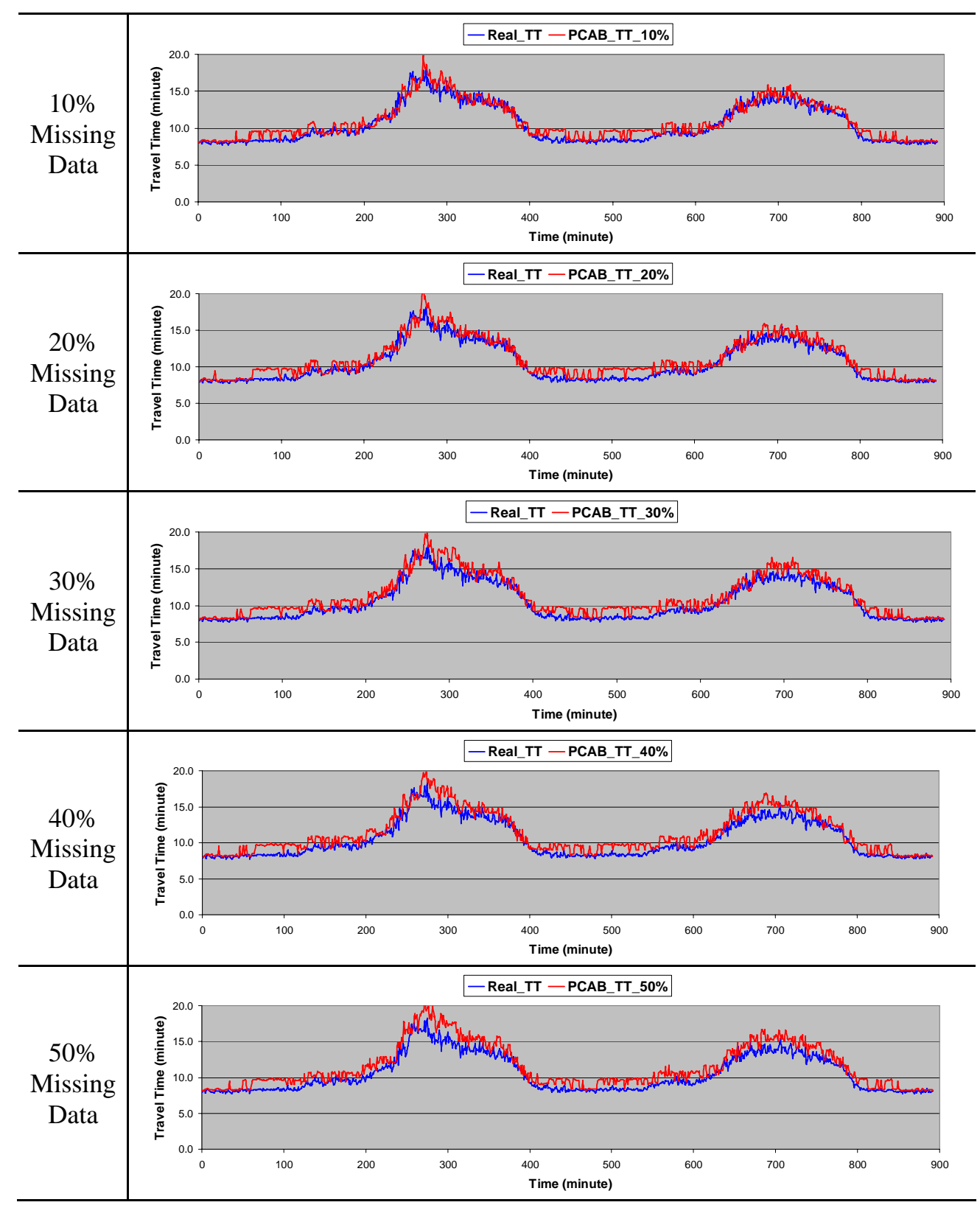

Note: PCAB_TT-xx\% means the PCAB Method Estimated Value with xx Percent Missing Data

Figure 4.5: Estimated Travel Time with Different Missing Data Percent 


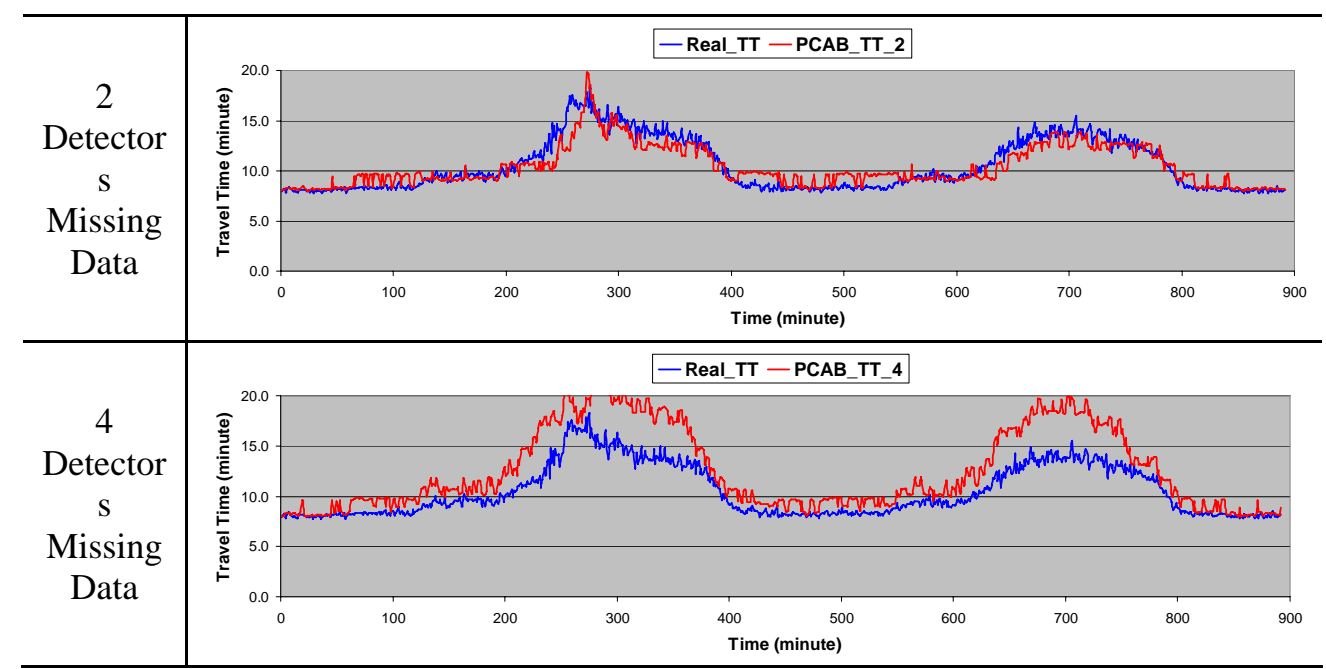

Note: PCAB_TT-x\% means the PCAB method estimated value with $\mathrm{x}$ number of malfunction detector.

Figure 4.6: Estimated Travel Time with Different Malfunction Detectors

\subsubsection{Validation of Speed Transformation Equation}

In the previous chapter, we have developed an equation to transfer time mean speed to space mean speed. The reason is based on previous research that the time mean speed is usually greater than the space mean speed. If the time mean speed is used in the estimation, this would have under-estimated the travel time. This is confirmed by the simulation data and the results are listed in Table 4.4 and Figure 4.7.

Table 4.4: Performance Measure of PLSB and PCAB Method with Time Mean Speed

\begin{tabular}{|c|c|c|c|}
\hline Rolling Step & Estimation Method & PLSB & PCAB \\
\hline \multirow{2}{*}{ One minute } & MAE (minutes) & 0.626 & 0.740 \\
\cline { 2 - 4 } & MAPE & $6.0 \%$ & $7.1 \%$ \\
\hline \multirow{2}{*}{ Two minutes } & MAE (minutes) & 0.619 & 0.735 \\
\cline { 2 - 4 } & MAPE & $5.9 \%$ & $7.0 \%$ \\
\hline \multirow{2}{*}{ Three minutes } & MAE (minutes) & 0.629 & 0.755 \\
\cline { 2 - 4 } & MAPE & $6.0 \%$ & $7.2 \%$ \\
\hline \multirow{2}{*}{ Four minutes } & MAE (minutes) & 0.640 & 0.740 \\
\cline { 2 - 4 } & MAPE & $6.1 \%$ & $7.1 \%$ \\
\hline \multirow{2}{*}{ Five minutes } & MAE (minutes) & 0.622 & 0.719 \\
\cline { 2 - 4 } & MAPE & $5.9 \%$ & $6.8 \%$ \\
\hline \multirow{2}{*}{ Average } & MAE (minutes) & 0.627 & 0.738 \\
\cline { 2 - 4 } & MAPE & $6.0 \%$ & $7.0 \%$ \\
\hline
\end{tabular}




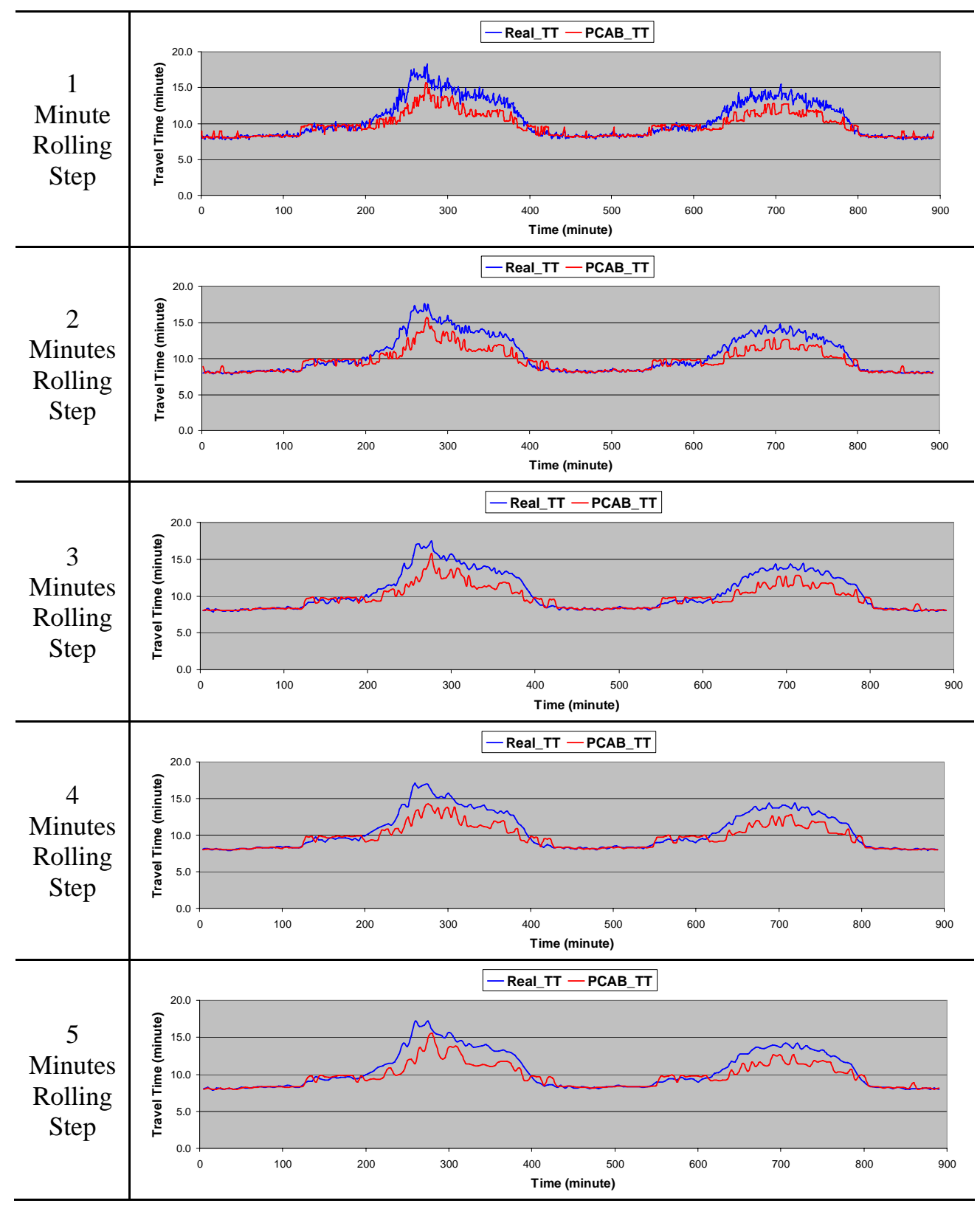

Figure 4.7: Estimated Travel Time by PCAB Comparing to Real Travel Time 


\section{CHAPTER 5}

FREEWAY TRAVAL TIME PREDICTION

\subsection{Introduction}

To be effective, the travel time information used for ATIS and ATMS applications should have two distinct characteristics: anticipative and dynamic. This is because every piece of information provided to users, to be useful, should yield predictions to the future and must be updated in real-time. In the FDOT Smart SunGuide software, instantaneous travel time is estimated with no prediction capability. Thus, there is a need for a method to more accurately and reliably predict travel times for the period that the vehicles are traveling on the road. This chapter investigated the travel time prediction problem.

Based on previous studies, NNs have improved to be an accurate and robust approach for travel time prediction. Dynamic Neural Networks (DNN) as a sub-class of NNs, are particularly suitable for travel time prediction. DNN take into account spatial and temporal information about traffic conditions simultaneously, and consider travel time from past time intervals as well as the current time interval when predicting travel time. However, DNN have not been adequately explored for travel time prediction. Based on the previous literature, only a sub-class of DNN called state-space neural networks (SSNN) has been used for travel time prediction (Van Lint et al. 2002; Van Lint,

2006; Liu, H. et al. 2006, Singh, and Abu-Lebdeh, 2007). Another important type of NNs referred to as time-delayed neural networks (TDNN) has been investigated for traffic volume prediction, but not for travel time prediction (Abdulhai, 1999; Lingras et al. 
2002; Ishak et al. 2003). In these studies, TDNN were shown to have the potential to be superior to more widely used NNs, such as the MLP, for traffic volume prediction.

This study explores the effectiveness of various topologies of DNN for travel time prediction. These include the TDNN, Generalized SSNN, and Fully RNN. The study compares the predictive qualities of these DNN structures with different parameter settings. In addition, this study compares the effectiveness of using tapped delay time memory versus Gamma memory as part of the three DNN structures for travel time prediction.

The qualities of the predicted travel times using the explored DNN were also compared with those obtained using a simple MLP, and also with the instantaneous travel times estimated for current traffic conditions with no attempt made to predict travel times for short-term future conditions. As stated earlier in this dissertation, most current realworld applications of ATIS and ATMS use instantaneous travel time estimation rather than travel time prediction.

In addition to the above, this study investigates the influences of two important parameters of travel time prediction. These are the rolling step, which defines the temporal resolution of input data in terms of the time interval at which the historical travel time data is aggregated for use in the analysis, and the prediction horizon, which defines the time window after which the prediction is made, usually set in the range of 0 15 minutes. The prediction horizon is used to account for situations in which the vehicles receiving the information will arrive at the point where the prediction is required at some time in the future. This may be, for example, the cases of information provided by traveler information web sites, hand-held devices, and in-vehicle devices. Thus, the 
prediction will need to be made starting at the time that these vehicles are expected to arrive at the point of prediction. With the prediction horizon of zero minutes, the model predicts the travel time starting at the current time. The use of a prediction horizon of zero is appropriate for the prediction of travel time displayed on Dynamic Message Signs (DMS) that provide travel time information to vehicles passing by the DMS.

\subsection{Dynamic Neural Networks}

Most of the NNs used in previous studies have used travel time information from the current time step to predict short-term future travel times. Due to the dynamic nature of traffic conditions, short-term future travel time may also be correlated with traffic conditions at previous time steps. Few of the studies have considered this correlation using NNs for travel time prediction as was described in the preceding section.

DNN comprise a class of neural networks that are particularly suitable for predicting variables such as travel time. A DNN model has a short-term memory, in which it stores previous outputs of the network or hidden neuron activities. Thus, it can account for traffic information from previous time steps in its prediction (Haykin, 1999). The DNN model receives input signals from one time instant (the current time instant) and combines these with signals from its memory to make predictions in a manner that is similar to the moving average approach in a time series modeling context. The advantages of this model are its straightforward design and the fact that the variations of traffic parameters with time are modeled implicitly. DNN vary in how the short-term memory is built and how it is applied to the neural network. 


\subsubsection{Short-term Memory}

For NNs to account for past and present conditions, it must be given a memory. The simple way of building a short-term memory into the structure of a neural network is through the use of time delays which can be implemented at the synaptic level inside the network or at the input layer of the network. Two types of delays have been used: tapped delay line memory (TM) and Gamma delay (GM). TM is the simplest and most commonly used form. With the help of the $Z$-transform, which is also called the unit delay operator, the current input signal can be transformed to a previous signal; that is, when $Z^{-1}$ operates on $x(t)$, it yields its delayed version $x(t-1)$. In Figure 5.1, the $Z^{-1}$ operator is applied $p$ times, resulting in $p$ unit delays (also called time lags). GM was first introduced by De Vries and Principe (1992). Figure 5.1 indicates that, with GM, the output of a junction has combined information from the current and the delayed inputs.

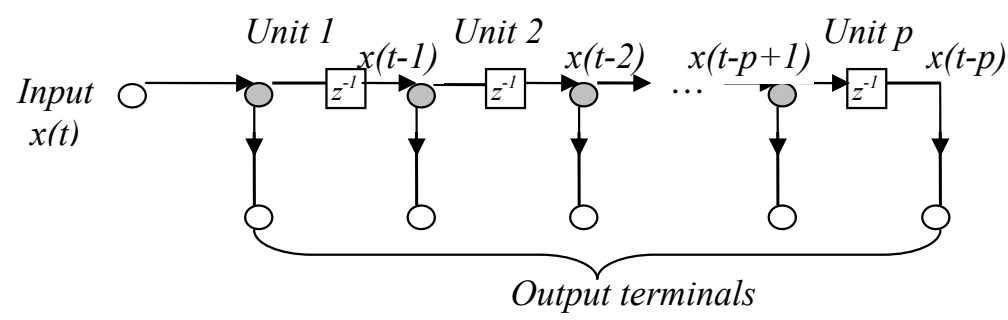

Tapped Delay Line Memory

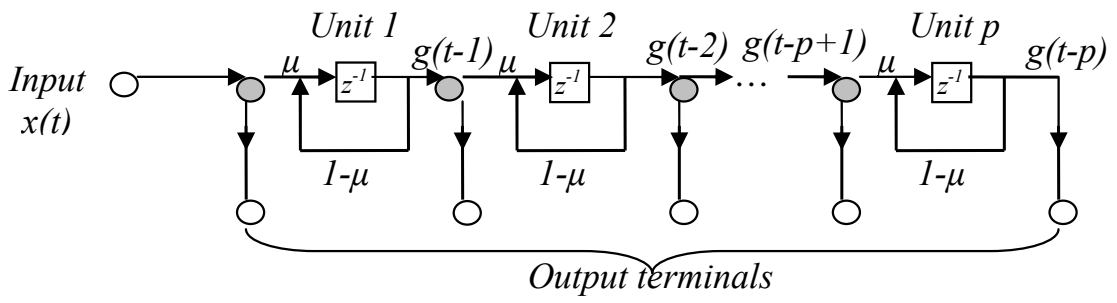

Gamma Memory

Figure 5.1: Short-term Memory Investigated in this Study 


\subsubsection{Feedback Application}

There are two basic ways of applying feedback to NNs: local feedback at the level of a single neuron inside the network and global feedback encompassing the whole network.
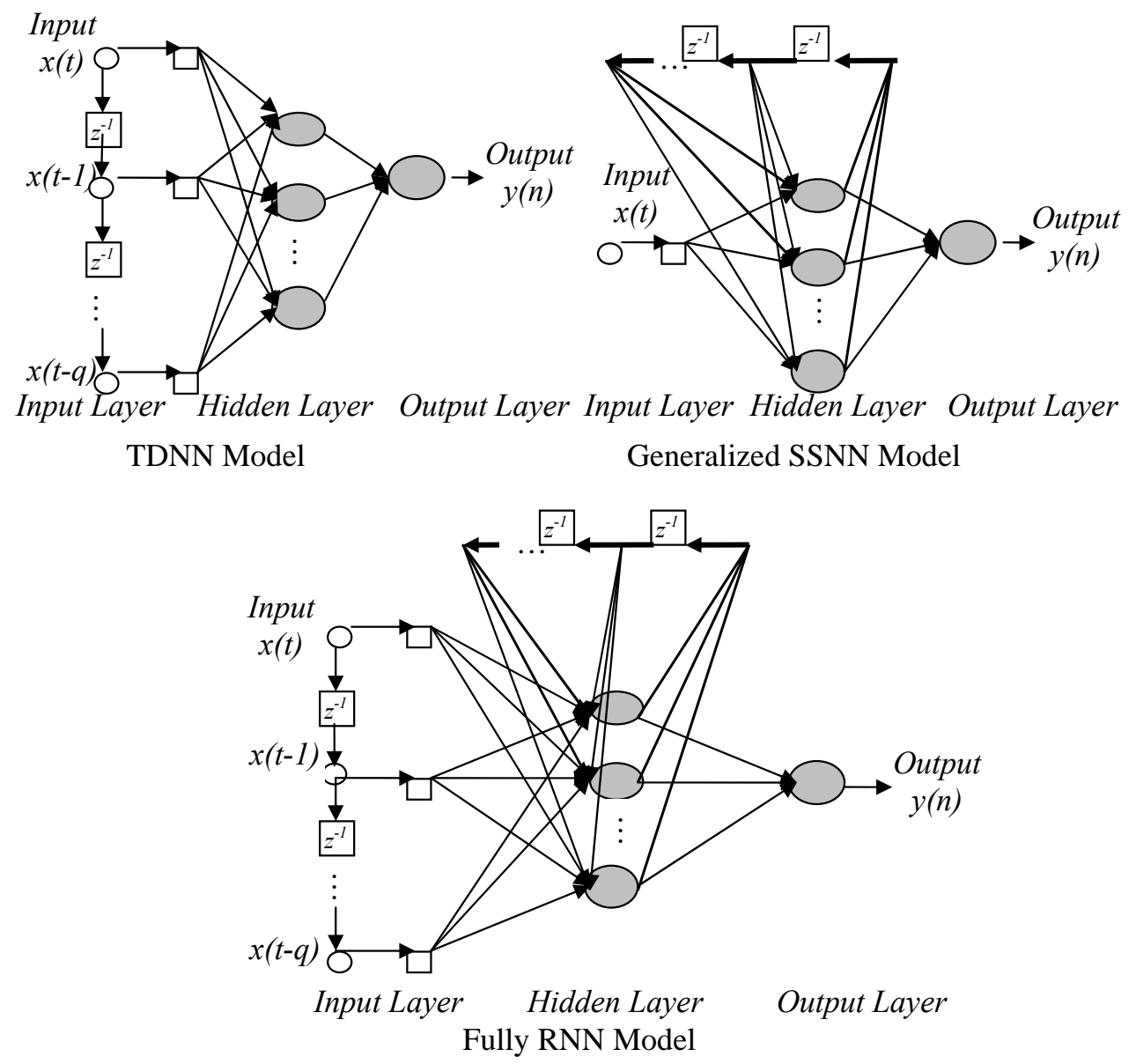

Figure 5.2: DNN Model Structure Investigated in this Study

Given a multilayer perceptron as the basic building block, local feedback can be applied to the input layer, at which the previous inputs are stored using the short-term memory and combined with the current instant input information. This is the TDNN 
network mentioned earlier in this paper. The NNs with global feedback is refereed to as a Recurrent Neural Network (RNN). The use of global feedback has the potential to reduce the memory requirement significantly compared to normal NNs solutions, in which the high dimensional parameter space is difficult to tackle.

The simplest RNN is the State-Space Neural Network (SSNN), which includes feedbacks from the hidden layer to the input layer. The SSNN model allows the inclusion of only one time lag memory of the hidden activities. However, a more generalized SSNN model allows variation in the number of time lags. This study investigates the use of both the TDNN and generalized SSNN models. In addition, this study investigates the use of a third structure refer to as the "fully RNN." This structure allows the implementation of a memory with varied time lags both on the input layer and the hidden layer. The TDNN, generalized SSNN, and fully RNN network structures are shown in Figure 5.2.

\subsection{Model Development}

The investigated NNs were trained using traffic detector data collected for the study segment between 6:00 AM and 9:00 PM. This period was selected because, for the remaining hours of the day, the traffic is light and free flow speed can be assumed. This study used three month's of data stored in the database for the months of June through August 2007. The June and July data were used for NN training, while August data were used for model testing. The DNN (with different structures and parameters) and MLP networks were trained and tested based on the same detector data. To investigate the effect of the length of the rolling step and prediction horizon, the training and testing of 
the MLP and different types of DNN were conducted separately for each aggregation rolling step and prediction horizon.

\subsubsection{Comparison of DNN and MLP}

The testing of the developed models was carried out first for the whole daytime period using data from three weekdays during the testing period. However, it was also decided to test the models separately for selected periods that exhibit considerable congestion compared to other periods during the testing period.

Four periods were identified that were particularly congested, as shown in Figure 5.4. The congested periods were from 3:02 PM to 6:04 PM of the first day, from 7:52 AM to 9:14 AM and from 12:30 PM to 6:45 PM of the second day and from 11:13 AM to 12:27 PM of the third day. Note that, based on the FDOT District 4 incident management database, lane blockage incidents occurred for the first three of the four peaks mentioned above. The duration of these lane blockages were 91, 32, and 59 minutes, respectively. The fourth peak also represents a non-recurrent congestion condition, although no incident could be identified based on the incident management database.

Conducting separate testing for the congested periods that include lane-blockage incidents allows for better examination of the performance of travel time prediction during these periods, which is more challenging and useful than the prediction in other time periods. 


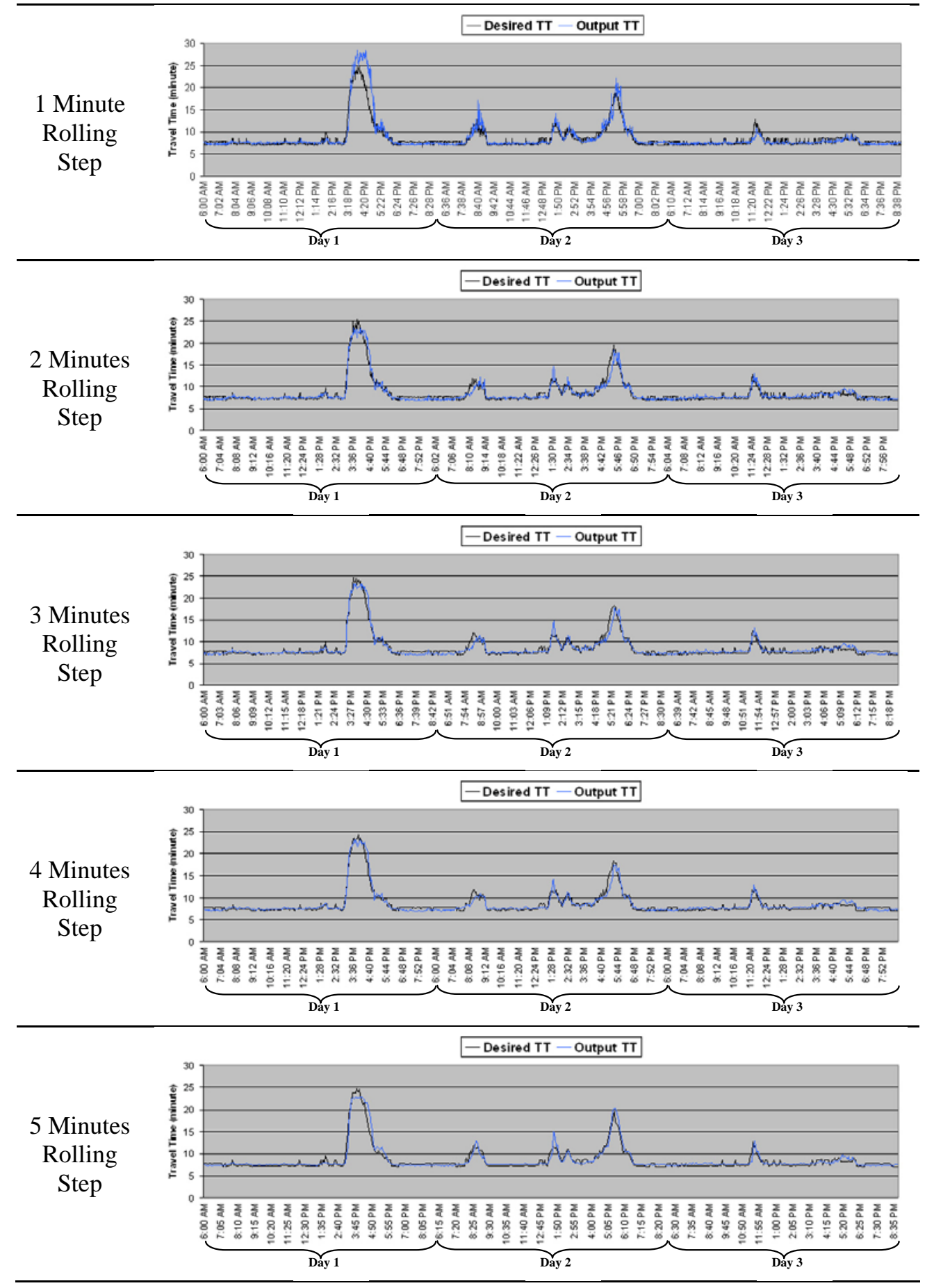

Figure 5.4: Travel Time Predicted by DNN Model in a Three-Day Period 
Figure 5.4 shows further comparison of the predicted and measured travel time data for the three weekdays with the four congested periods, the "desired TT" is the target travel time, which is the measured travel time, the "Output TT" is that predicted by the DNN. As can be seen, the tested DNN models were able to predict travel time well in all investigated flow conditions, particularly with the 3-to-4-minute rolling step, the predicted travel time for a 1-minute rolling step is not as good as that for longer rolling steps.

Figure 5.5 shows the performance of travel time prediction for the different types of DNN and MLP for different rolling steps, compared to the instantaneous travel time estimated based on the assumption of unchanged traffic conditions. The results presented in Figure 5.5 are for a prediction horizon of zero minutes. The MLP and the DNN models had only one hidden layer. The results presented for the different DNN models were the best results obtained for each model, when varying the parameters settings, as discussed in the next section.
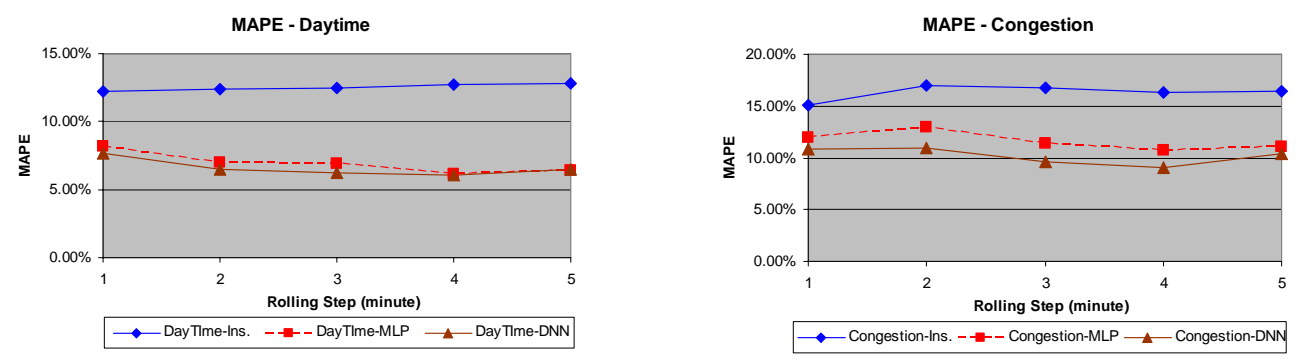

Figure 5.5: MAPE of DNN, MLP and Instantaneous Estimation

The results presented in Figure 5.5 show that the predictions using the DNN and MLP outperform the instantaneous travel time estimation in predicting short-term future travel time. The MAPE was reduced by $40 \%$ for predicted travel time for daytime 
conditions and was reduced by about $25 \%$ for the congested conditions, compared to the instantaneous travel time. The performance of the travel time prediction during the congested conditions of all tested networks was worse in incident/congested conditions than during daytime conditions.

Figure 5.6 shows that the best DNN setup works better than the MLP, particularly for congested/incident conditions and rolling steps of 2, 3, and 4 minutes.

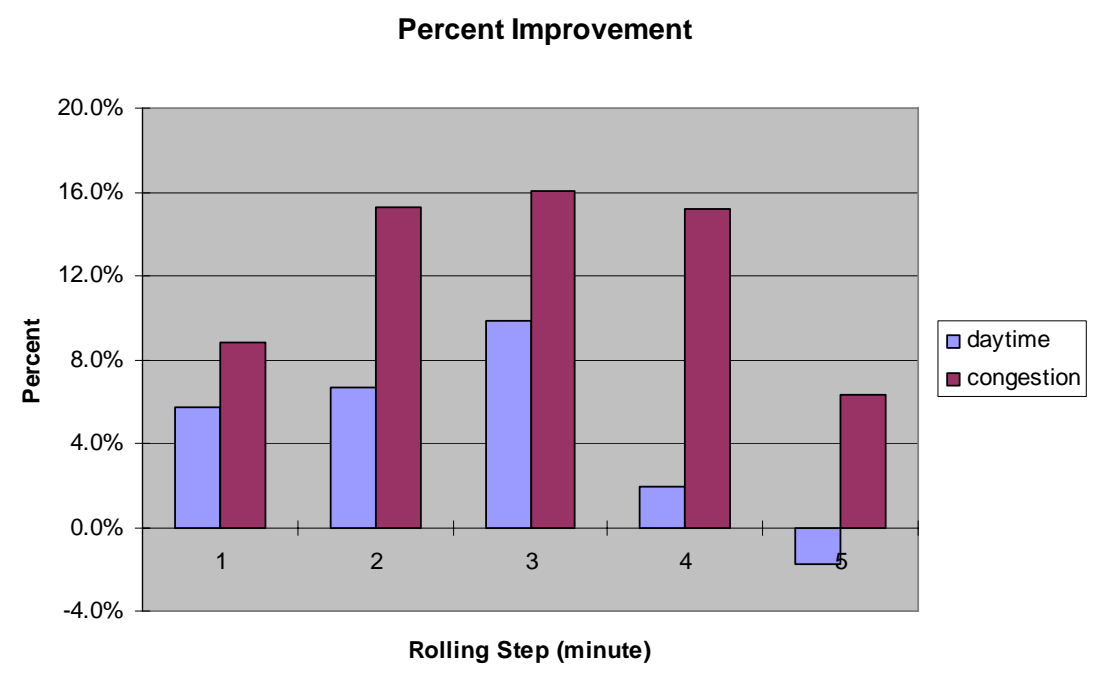

Figure 5.6: Performance Improvement of DNN Compared to MLP

More specific percent improvement is listed in Table 5.1, up to a $16 \%$ improvement in the prediction as reflected by the MAPE value for daytime conditions and a 10\% improvement for congested conditions was achieved with the DNN prediction compared to the MLP prediction. 
Table 5.1: Performance of MLP and DNN models

\begin{tabular}{|c|c|c|c|c|c|c|c|}
\hline \multirow{3}{*}{$\begin{array}{l}\text { Rolling } \\
\text { Step }\end{array}$} & \multicolumn{6}{|c|}{ Daytime (6:00 AM 9:00 PM) } & \multirow{3}{*}{$\begin{array}{c}\text { DNN Compare } \\
\text { To MLP } \\
\text { Improvement }\end{array}$} \\
\hline & \multicolumn{2}{|c|}{ Instantaneous } & \multicolumn{2}{|c|}{ MLP } & \multicolumn{2}{|c|}{ DNN Model } & \\
\hline & MAE & MAPE & MAE & MAPE & MAE & MAPE & \\
\hline 1 minute & 1.05 & $12.2 \%$ & 0.7 & $8.17 \%$ & 0.66 & $7.70 \%$ & $5.7 \%$ \\
\hline 2 minutes & 1.07 & $12.4 \%$ & 0.6 & $7.00 \%$ & 0.56 & $6.53 \%$ & $6.7 \%$ \\
\hline 3 minutes & 1.07 & $12.5 \%$ & 0.59 & $6.88 \%$ & 0.53 & $6.21 \%$ & $9.9 \%$ \\
\hline 4 minutes & 1.09 & $12.7 \%$ & 0.53 & $6.18 \%$ & 0.52 & $6.07 \%$ & $1.9 \%$ \\
\hline \multirow[t]{2}{*}{5 minutes } & 1.10 & $12.8 \%$ & 0.55 & $6.42 \%$ & 0.56 & $6.53 \%$ & $-1.8 \%$ \\
\hline & \multicolumn{6}{|c|}{ Congestion Time } & \\
\hline 1 minute & 1.30 & $15.1 \%$ & 1.02 & $11.90 \%$ & 0.93 & $10.85 \%$ & $8.8 \%$ \\
\hline 2 minutes & 1.46 & $17.0 \%$ & 1.11 & $12.95 \%$ & 0.94 & $10.97 \%$ & $15.3 \%$ \\
\hline 3 minutes & 1.44 & $16.8 \%$ & 0.98 & $11.44 \%$ & 0.82 & $9.60 \%$ & $16.0 \%$ \\
\hline 4 minutes & 1.40 & $16.3 \%$ & 0.92 & $10.74 \%$ & 0.78 & $9.10 \%$ & $15.2 \%$ \\
\hline 5 minutes & 1.40 & $16.4 \%$ & 0.95 & $11.09 \%$ & 0.89 & $10.39 \%$ & $6.3 \%$ \\
\hline
\end{tabular}

Note: Unit of MAE is minute

\subsubsection{Effect of DNN Network Topology}

Tables 5.2a-5.2e show the results of a comparison of the three DNN structures investigated in this study: the TDNN, Generalized SSNN, and Fully RNN. In addition, Table 5.1 includes the results using the two types of memories investigated in this study (the TM and the GM). The results presented in Table 5.2(a,b,c,d,e) are for a zero-minute prediction horizon. Because the performance trend is similar for the five rolling steps investigated in this study, only the results of the1- and 4-minute rolling steps are presented in this paper. 
Table 5.2a: Prediction Results of Different DNN Models Compared to MLP Model

\begin{tabular}{|c|c|c|c|c|c|}
\hline \multirow{3}{*}{$\begin{array}{c}\text { Rolling } \\
\text { Step }\end{array}$} & \multirow{2}{*}{ Model } & \multicolumn{2}{c|}{ Daytime (6AM 9PM) } & \multicolumn{2}{c|}{ Congestion Time } \\
\cline { 2 - 6 } & & MAE & MAPE & MAE & MAPE \\
\hline \multirow{4}{*}{ MLP } & 0.70 & $8.2 \%$ & 1.02 & $11.9 \%$ \\
\cline { 2 - 6 } & TDNN - TM - 1 lag & 0.66 & $7.8 \%$ & 0.93 & $10.9 \%$ \\
\cline { 2 - 6 } & TDNN - TM - 2 lag & 0.56 & $6.6 \%$ & 1.40 & $16.4 \%$ \\
\cline { 2 - 6 } & TDNN - TM - 3 lag & 0.55 & $6.4 \%$ & 1.32 & $15.4 \%$ \\
\cline { 2 - 6 } & TDNN - GM - 1 lag & 0.51 & $6.0 \%$ & 0.95 & $11.0 \%$ \\
\cline { 2 - 6 } & TDNN - GM - 2 lag & 0.50 & $5.8 \%$ & 1.07 & $12.4 \%$ \\
\cline { 2 - 6 } & TDNN - GM - 3 lag & 0.46 & $5.4 \%$ & 1.08 & $12.6 \%$ \\
\cline { 2 - 6 } & Generalized SSNN - 1 lag & 0.58 & $6.7 \%$ & 1.15 & $13.4 \%$ \\
\cline { 2 - 6 } & Generalized SSNN - 2 lag & 0.58 & $6.8 \%$ & 1.27 & $14.9 \%$ \\
\cline { 2 - 6 } & Fully RNN+TM-1 lag & 0.53 & $6.2 \%$ & 1.25 & $14.6 \%$ \\
\cline { 2 - 6 } & Fully RNN+TM-2 lag & 0.71 & $8.3 \%$ & 1.42 & $16.6 \%$ \\
\cline { 2 - 6 } & Fully RNN+GM-1 lag & 0.70 & $8.1 \%$ & 1.23 & $14.4 \%$ \\
\cline { 2 - 6 } & Fully RNN+GM-2 lag & 0.79 & $9.2 \%$ & 1.06 & $12.3 \%$ \\
\cline { 2 - 6 } & & & & &
\end{tabular}

Note: Unit of MAE is minute.

Table 5.2b: Prediction Results of Different DNN Models Compared to MLP Model

\begin{tabular}{|c|c|c|c|c|c|}
\hline \multirow{3}{*}{$\begin{array}{c}\text { Rolling } \\
\text { Step }\end{array}$} & \multirow{2}{*}{ Model } & \multicolumn{2}{c|}{ Daytime (6AM 9PM) } & \multicolumn{2}{c|}{ Congestion Time } \\
\cline { 2 - 6 } & & MAE & MAPE & MAE & MAPE \\
\hline \multirow{4}{*}{ MLP } & 0.6 & $7.0 \%$ & 1.11 & $13.0 \%$ \\
\cline { 2 - 6 } & TDNN - TM - 1 lag & 0.56 & $6.6 \%$ & 0.94 & $11.0 \%$ \\
\cline { 2 - 6 } & TDNN - TM - 2 lag & 0.76 & $8.9 \%$ & 1.71 & $20.0 \%$ \\
\cline { 2 - 6 } & TDNN - TM - 3 lag & 0.72 & $8.4 \%$ & 1.38 & $16.1 \%$ \\
\cline { 2 - 6 } & TDNN - GM - 1 lag & 0.65 & $7.5 \%$ & 1.19 & $13.9 \%$ \\
\cline { 2 - 6 } & TDNN - GM - 2 lag & 0.68 & $8.0 \%$ & 1.26 & $14.7 \%$ \\
\cline { 2 - 6 } & TDNN - GM - 3 lag & 0.62 & $7.3 \%$ & 1.28 & $14.9 \%$ \\
\cline { 2 - 6 } & Generalized SSNN - 1 lag & 0.70 & $8.2 \%$ & 1.50 & $17.5 \%$ \\
\cline { 2 - 6 } & Generalized SSNN - 2 lag & 0.75 & $8.7 \%$ & 1.50 & $17.5 \%$ \\
\cline { 2 - 6 } & Fully RNN+TM -1 lag & 0.72 & $8.3 \%$ & 1.55 & $18.1 \%$ \\
\cline { 2 - 6 } & Fully RNN+TM -2 lag & 0.79 & $9.2 \%$ & 1.40 & $16.3 \%$ \\
\cline { 2 - 6 } & Fully RNN+GM -1 lag & 0.67 & $7.8 \%$ & 1.21 & $14.1 \%$ \\
\cline { 2 - 6 } & Fully RNN+GM -2 lag & 0.75 & $8.8 \%$ & 1.72 & $20.1 \%$ \\
\hline
\end{tabular}

Note: Unit of MAE is minute. 
Table 5.2c: Prediction Results of Different DNN Models Compared to MLP Model

\begin{tabular}{|c|c|c|c|c|c|}
\hline \multirow{3}{*}{$\begin{array}{c}\text { Rolling } \\
\text { Step }\end{array}$} & \multirow{2}{*}{ Model } & \multicolumn{2}{|c|}{ Daytime (6AM 9PM) } & \multicolumn{2}{c|}{ Congestion Time } \\
\cline { 2 - 6 } & & MAE & MAPE & MAE & MAPE \\
\hline \multirow{5}{*}{ MLP } & 0.59 & $6.9 \%$ & 0.98 & $11.4 \%$ \\
\cline { 2 - 6 } & TDNN - TM - 1 lag & 0.53 & $6.2 \%$ & 0.82 & $9.6 \%$ \\
\cline { 2 - 6 } & TDNN - TM - 2 lag & 0.57 & $6.7 \%$ & 0.99 & $11.6 \%$ \\
\cline { 2 - 6 } & TDNN - GM - 1 lag & 0.65 & $7.6 \%$ & 1.36 & $15.9 \%$ \\
\cline { 2 - 6 } & TDNN - GM - 2 lag & 0.61 & $7.1 \%$ & 1.28 & $15.0 \%$ \\
\cline { 2 - 6 } & Generalized SSNN - 1 lag & 0.69 & $8.0 \%$ & 1.19 & $13.9 \%$ \\
\cline { 2 - 6 } & Generalized SSNN - 2 lag & 0.71 & $8.3 \%$ & 1.53 & $17.9 \%$ \\
\cline { 2 - 6 } & Fully RNN+TM -1 lag & 0.77 & $9.0 \%$ & 1.60 & $18.7 \%$ \\
\cline { 2 - 6 } & Fully RNN+TM -2 lag & 0.81 & $9.4 \%$ & 1.38 & $16.1 \%$ \\
\cline { 2 - 6 } & Fully RNN+GM -1 lag & 0.70 & $8.1 \%$ & 1.51 & $17.6 \%$ \\
\cline { 2 - 6 } & Fully RNN+GM -2 lag & 0.76 & $8.9 \%$ & 1.29 & $15.0 \%$ \\
\hline
\end{tabular}

Note: Unit of MAE is minute.

Table 5.2d: Prediction Results of Different DNN Models Compared to MLP Model

\begin{tabular}{|c|c|c|c|c|c|}
\hline \multirow{3}{*}{$\begin{array}{c}\text { Rolling } \\
\text { Step }\end{array}$} & \multirow{2}{*}{ Model } & \multicolumn{2}{c|}{ Daytime (6AM 9PM) } & \multicolumn{2}{c|}{ Congestion Time } \\
\cline { 2 - 6 } & & MAE & MAPE & MAE & MAPE \\
\hline \multirow{5}{*}{ MLP } & 0.53 & $6.2 \%$ & 0.92 & $10.7 \%$ \\
\cline { 2 - 6 } & TDNN - TM - 1 lag & 0.52 & $6.0 \%$ & 0.78 & $9.1 \%$ \\
\cline { 2 - 6 } & TDNN - TM - 2 lag & 0.70 & $8.1 \%$ & 1.28 & $14.9 \%$ \\
\cline { 2 - 6 } & TDNN - GM - 1 lag & 0.56 & $6.5 \%$ & 1.08 & $12.6 \%$ \\
\cline { 2 - 6 } & TDNN - GM - 2 lag & 0.59 & $6.8 \%$ & 1.11 & $13.0 \%$ \\
\cline { 2 - 6 } & Generalized SSNN - 1 lag & 0.67 & $7.8 \%$ & 1.46 & $17.1 \%$ \\
\cline { 2 - 6 } & Generalized SSNN - 2 lag & 0.72 & $8.5 \%$ & 1.33 & $15.5 \%$ \\
\cline { 2 - 6 } & Fully RNN+TM -1 lag & 0.69 & $8.0 \%$ & 1.39 & $16.3 \%$ \\
\cline { 2 - 6 } & Fully RNN+TM -2 lag & 0.69 & $8.0 \%$ & 1.35 & $15.7 \%$ \\
\cline { 2 - 6 } & Fully RNN+GM -1 lag & 0.74 & $8.7 \%$ & 1.53 & $17.9 \%$ \\
\cline { 2 - 6 } & Fully RNN+GM - lag & 0.76 & $8.9 \%$ & 1.55 & $18.1 \%$ \\
\hline
\end{tabular}

Note: Unit of MAE is minute. 
Table 5.2e: Prediction Results of Different DNN Models Compared to MLP Model

\begin{tabular}{|c|c|c|c|c|c|}
\hline \multirow{3}{*}{$\begin{array}{c}\text { Rolling } \\
\text { Step }\end{array}$} & \multirow{2}{*}{ Model } & \multicolumn{2}{c|}{ Daytime (6AM 9PM) } & \multicolumn{2}{c|}{ Congestion Time } \\
\cline { 2 - 6 } & & MAE & MAPE & MAE & MAPE \\
\hline \multirow{5}{*}{ MLP } & 0.55 & $6.42 \%$ & 0.95 & $11.1 \%$ \\
\cline { 2 - 6 } & TDNN - TM - 1 lag & 0.61 & $7.2 \%$ & 1.18 & $13.8 \%$ \\
\cline { 2 - 6 } & TDNN - TM - 2 lag & 0.74 & $8.6 \%$ & 1.54 & $18.0 \%$ \\
\cline { 2 - 6 } & TDNN - GM - 1 lag & 0.61 & $7.1 \%$ & 1.01 & $11.7 \%$ \\
\cline { 2 - 6 } & TDNN - GM - 2 lag & 0.56 & $6.5 \%$ & 0.89 & $10.3 \%$ \\
\cline { 2 - 6 } & Generalized SSNN - 1 lag & 0.65 & $7.6 \%$ & 1.27 & $14.8 \%$ \\
\cline { 2 - 6 } & Generalized SSNN - 2 lag & 0.72 & $8.4 \%$ & 1.51 & $17.6 \%$ \\
\cline { 2 - 6 } & Fully RNN+TM -1 lag & 0.64 & $7.4 \%$ & 1.34 & $15.7 \%$ \\
\cline { 2 - 6 } & Fully RNN+TM -2 lag & 0.68 & $8.0 \%$ & 1.59 & $18.6 \%$ \\
\cline { 2 - 6 } & Fully RNN+GM - 1 lag & 0.64 & $7.4 \%$ & 1.04 & $12.2 \%$ \\
\cline { 2 - 6 } & Fully RNN+GM -2 lag & 0.70 & $8.1 \%$ & 1.36 & $15.9 \%$ \\
\hline
\end{tabular}

Note: Unit of MAE is minute.

Tables 5.2a-5.2e indicate that MLP can perform close to the best DNN. This indicates that the travel time estimated at the current time step is an important determining factor for future travel time prediction. However, the TDNN model was found to perform better than the MLP model for all aggregation time intervals. This means that the use of the immediate past traffic conditions is also correlated with the future travel time and can improve travel time prediction accuracy.

The TDNN model with tapped delay line memory worked best in all the models. The TDNN model with local feedback worked better than the generalized SSNN model with global feedback and the fully RNN that includes both local and global feedback. In most cases, the TDNN models worked best with only one time lag. Thus, it can be concluded that only the very near past information can be useful for prediction. The best performance was achieved when a TDNN was used with one time lag and a 4-minute rolling step (MAPE equal to 9.1\%). The best performance achieved with the MLP was also with the 4-minute rolling step (MAPE equal to 10.7\%). 


\subsection{Effect of Prediction Horizon}

Based on the results presented in preceding section, two types of NNs structures were selected for further investigation of the effects of the variation of the prediction horizon on the prediction quality. They are the MLP and the TDNN with tapped delay line memory. The results are list in Tables 5.3a and 5.3b and more visual plot is shown in Figure 5.7.

Table 5.3a: Model Performance with Varied Prediction Horizon for Daytime

\begin{tabular}{|c|c|c|c|c|c|c|}
\hline \multirow{3}{*}{$\begin{array}{l}\text { Rolling } \\
\text { Step }\end{array}$} & \multirow{3}{*}{$\begin{array}{c}\text { Prediction } \\
\text { Horizon }\end{array}$} & \multicolumn{5}{|c|}{ Daytime (6AM 9PM) } \\
\hline & & \multicolumn{2}{|c|}{ MLP } & \multicolumn{2}{|c|}{ TDNN } & \multirow{2}{*}{ Improvement } \\
\hline & & MAE & MAPE & MAE & MAPE & \\
\hline \multirow{4}{*}{$\begin{array}{c}1 \\
\text { minute }\end{array}$} & Current & 0.70 & $8.2 \%$ & 0.66 & $7.8 \%$ & $5.4 \%$ \\
\hline & 5 minutes & 0.81 & $9.4 \%$ & 0.71 & $8.3 \%$ & $11.6 \%$ \\
\hline & 10 minutes & 0.85 & $10.0 \%$ & 0.98 & $11.5 \%$ & $-15.0 \%$ \\
\hline & 15 minutes & 0.97 & $11.4 \%$ & 0.96 & $11.2 \%$ & $1.1 \%$ \\
\hline \multirow{4}{*}{$\begin{array}{c}2 \\
\text { minutes }\end{array}$} & Current & 0.60 & $6.9 \%$ & 0.56 & $6.6 \%$ & $5.5 \%$ \\
\hline & 4 minutes & 0.68 & $8.0 \%$ & 0.70 & $8.1 \%$ & $-1.8 \%$ \\
\hline & 10 minutes & 0.77 & $9.0 \%$ & 0.84 & $9.8 \%$ & $-8.8 \%$ \\
\hline & 16 minutes & 0.87 & $10.2 \%$ & 1.00 & $11.7 \%$ & $-15.2 \%$ \\
\hline \multirow{4}{*}{$\begin{array}{c}3 \\
\text { minutes }\end{array}$} & Current & 0.59 & $6.9 \%$ & 0.53 & $6.2 \%$ & $10.3 \%$ \\
\hline & 6 minutes & 0.70 & $8.2 \%$ & 0.83 & $9.7 \%$ & $-19.4 \%$ \\
\hline & 9 minutes & 0.76 & $8.9 \%$ & 0.79 & $9.2 \%$ & $-3.3 \%$ \\
\hline & 15 minutes & 0.91 & $10.7 \%$ & 1.00 & $11.7 \%$ & $-9.4 \%$ \\
\hline \multirow{4}{*}{$\begin{array}{c}4 \\
\text { minutes }\end{array}$} & Current & 0.53 & $6.2 \%$ & 0.52 & $6.0 \%$ & $2.1 \%$ \\
\hline & 4 minutes & 0.67 & $7.8 \%$ & 0.75 & $8.8 \%$ & $-12.0 \%$ \\
\hline & 8 minutes & 0.74 & $8.7 \%$ & 0.75 & $8.8 \%$ & $-1.5 \%$ \\
\hline & 16 minutes & 0.86 & $10.0 \%$ & 0.94 & $11.0 \%$ & $-9.5 \%$ \\
\hline \multirow{4}{*}{$\begin{array}{c}5 \\
\text { minutes }\end{array}$} & Current & 0.55 & $6.4 \%$ & 0.56 & $6.5 \%$ & $-0.9 \%$ \\
\hline & 5 minutes & 0.72 & $8.4 \%$ & 0.62 & $7.3 \%$ & $13.6 \%$ \\
\hline & 10 minutes & 0.75 & $8.8 \%$ & 0.82 & $9.6 \%$ & $-9.2 \%$ \\
\hline & 15 minutes & 0.83 & $9.7 \%$ & 0.96 & $11.2 \%$ & $-16.0 \%$ \\
\hline
\end{tabular}

Note: Unit of MAE is minute. 
Table 5.3b: Model Performance with Varied Prediction Horizon for Congestion Time

\begin{tabular}{|c|c|c|c|c|c|c|}
\hline \multirow{3}{*}{$\begin{array}{c}\text { Rolling } \\
\text { Step }\end{array}$} & \multirow{3}{*}{$\begin{array}{c}\text { Prediction } \\
\text { Horizon }\end{array}$} & \multicolumn{5}{|c|}{ Congestion Time } \\
\hline & & \multicolumn{2}{|c|}{ MLP } & \multicolumn{2}{|c|}{ TDNN } & \multirow{2}{*}{ Improvement } \\
\hline & & MAE & MAPE & MAE & MAPE & \\
\hline \multirow{4}{*}{$\begin{array}{c}1 \\
\text { minute }\end{array}$} & Current & 1.02 & $11.9 \%$ & 0.93 & $10.9 \%$ & $8.1 \%$ \\
\hline & 5 minutes & 1.60 & $18.7 \%$ & 1.37 & $16.0 \%$ & $14.5 \%$ \\
\hline & 10 minutes & 1.83 & $21.3 \%$ & 1.73 & $20.2 \%$ & $5.1 \%$ \\
\hline & 15 minutes & 2.11 & $24.6 \%$ & 2.12 & $24.8 \%$ & $-0.6 \%$ \\
\hline \multirow{4}{*}{$\begin{array}{c}2 \\
\text { minutes }\end{array}$} & Current & 1.11 & $12.9 \%$ & 0.94 & $11.0 \%$ & $15.0 \%$ \\
\hline & 4 minutes & 1.36 & $15.8 \%$ & 1.33 & $15.5 \%$ & $1.7 \%$ \\
\hline & 10 minutes & 1.68 & $19.7 \%$ & 2.04 & $23.8 \%$ & $-21.0 \%$ \\
\hline & 16 minutes & 2.01 & $23.5 \%$ & 2.06 & $24.0 \%$ & $-2.2 \%$ \\
\hline \multirow{4}{*}{$\begin{array}{c}3 \\
\text { minutes }\end{array}$} & Current & 0.98 & $11.4 \%$ & 0.82 & $9.6 \%$ & $15.8 \%$ \\
\hline & 6 minutes & 1.35 & $15.8 \%$ & 1.59 & $18.6 \%$ & $-17.6 \%$ \\
\hline & 9 minutes & 1.58 & $18.5 \%$ & 1.79 & $20.9 \%$ & $-13.1 \%$ \\
\hline & 15 minutes & 2.07 & $24.1 \%$ & 2.14 & $25.0 \%$ & $-3.7 \%$ \\
\hline \multirow{4}{*}{$\begin{array}{c}4 \\
\text { minutes }\end{array}$} & Current & 0.92 & $10.7 \%$ & 0.78 & $9.1 \%$ & $15.3 \%$ \\
\hline & 4 minutes & 1.37 & $16.0 \%$ & 1.25 & $14.6 \%$ & $8.5 \%$ \\
\hline & 8 minutes & 1.55 & $18.1 \%$ & 1.61 & $18.8 \%$ & $-3.9 \%$ \\
\hline & 16 minutes & 2.00 & $23.4 \%$ & 1.96 & $22.9 \%$ & $2.1 \%$ \\
\hline \multirow{4}{*}{$\begin{array}{c}5 \\
\text { minutes }\end{array}$} & Current & 0.95 & $11.1 \%$ & 0.89 & $10.3 \%$ & $6.6 \%$ \\
\hline & 5 minutes & 1.31 & $15.3 \%$ & 1.13 & $13.2 \%$ & $13.4 \%$ \\
\hline & 10 minutes & 1.48 & $17.2 \%$ & 1.57 & $18.4 \%$ & $-6.6 \%$ \\
\hline & 15 minutes & 1.84 & $21.4 \%$ & 2.17 & $25.3 \%$ & $-18.1 \%$ \\
\hline
\end{tabular}

Note: Unit of MAE is minute.

It can be concluded that the prediction accuracy decreases as the prediction horizon increases, particularly for short rolling steps and congested/incident conditions. The TDNN model does not out-perform the MLP model for longer prediction horizons. 

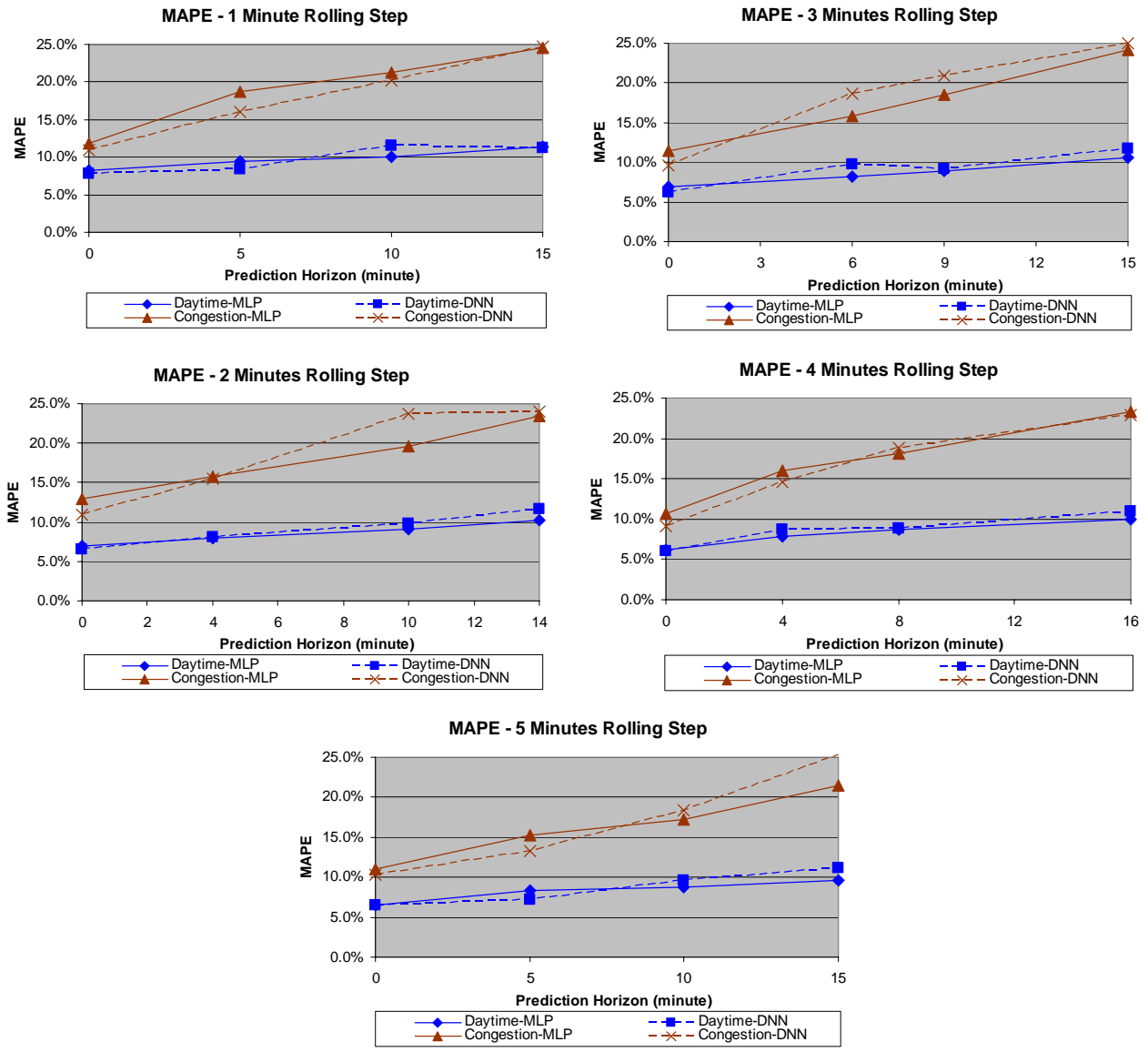

Figure 5.7: MAPE with Varied Prediction Horizons 


\section{CHAPTER 6}

\section{CONCLUSIONS AND RECOMMENDATIONS}

This dissertation study has accomplished the following major research tasks: (1) identifying data imputation methods to deal with missing traffic detector data and examined the effectiveness of the speed transformation equations, (2) identifying and testing travel time estimation methods based on traffic detector data, (3) developing a data-driven travel time prediction model and testing the performance of the developed model, and (4) examining the influence of different Dynamic Neural Network (DNN) model structures and parameters on the performance of the travel time prediction model.

\subsection{Summary of Research Results and Conclusions}

\section{(1) Data Preprocessing}

A time and space interpolation method was selected to deal with random missing detector data and structure detector malfunction for off-line travel time prediction model training. For on-line model application a method of Exponential Moving Average (EMA) method is used replacing the time interpolation method because the future measurement is not available. In addition, two linear regression models were developed for high and low speed regions to transform time mean speed to space mean speed. Based on simulation data, both the data imputation and data transformation methods identified in this study were validated and proved to be effective. 
(2) Travel Time Estimation

A new trajectory method referenced to as the Piecewise Constant Acceleration Based (PCAB) method was proposed in this study. Tested with the simulation data, one of the constant speed trajectory methods (the average speed method) was found to perform almost as good as the proposed PCAB method. Both of these methods perform better than the other investigated trajectory methods.

\section{(3) Travel Time Prediction}

Based on the results presented in this study, it can be concluded that both the Time Delay Neural Networks (TDNN) and Multilayer Perceptron (MLP) were able to produce better predictions of travel time compared to the use of instantaneous travel time. The prediction results in increasing the accuracy of travel time by about $40 \%$ for the whole daytime conditions and by $25 \%$ for congested peak periods.

The TDNN topology with two, three, and four minute rolling steps and tapped delay time memory was found to out-perform various investigated configurations of MLP, Generalized State Space Neural Networks (SSNN), and Fully Recurrent Neural Networks (RNN). However, the improvement in the prediction with the use of TDNN compared to the use of MLP was relatively small for the investigated transportation system. The better performance of TDNN compared to Generalized SSNN and Fully RNN indicates that local feedback works better than global feedback.

TDNN with only one time lag performed the best among the investigated time lags, indicating that only the information from the immediate past time step is useful for time prediction. Based on the results of this study, it can be also concluded that the 
prediction accuracy decreases as the prediction horizon increases, especially for shorter aggregation periods and congested conditions.

\subsection{Recommendations for Future Development}

Although this study has achieved the proposed objectives, much research remains to be done. Some directions for future research are given below.

The findings regarding the best NNs topology and parameters as discussed above may be affected by the size of the system under consideration. For example, if data are available from detectors located long distances upstream of the segment for which travel time prediction is needed, the information from previous time lags will be more relevant to the estimation and the DNN structures are expected to become more effective compared to MLP. It is recommended that an additional investigation be made of the effect of the size of the network and the location of upstream detectors on travel time prediction to determine how these factors will affect the conclusions from this study.

In this study, only traffic detector data were used in the prediction. There is a need to further explore the combination of detector and incident data in the prediction of travel time. By including incident attributes as an input to the prediction, it is expected that improvements in travel time prediction can be achieved.

In this study, only real-time data were used as an input to the model training and testing. It is possible that combining the real-time data with historical data that represent the average conditions on the corridor at a given time interval may further improve the quality of prediction. 
The methodology presented in this study can be classified as a data-driven method. It is recommended that an evaluation be done of model-based and data driven methods to decide on conditions for which each type of prediction is more appropriate.

In this study, a threshold was set of the acceptable error in travel time estimation without further analysis of the effect of this threshold on travel time prediction. It is recommended that this effect should be evaluated in future studies. 


\section{REFERENCES}

Abdulhai, B., Porwal, H., and Recker, W., "Short-Term Freeway Traffic Flow Prediction Using Genetically-Optimized Time-Delay-Based Neural Networks”, TRB 1999 Annual Meeting CD-ROM, 1999.

Ahmed, M. S., and Cook, A. R., "Analysis of Freeway Traffic Time-Series Data by Using Box-Jenkins Techniques”, Transportation Research Record, 722, Transportation Research Board, Washington, DC, pp. 1-9, 1979.

Armitage, W., and Lo, J.-C., "Enhancing The Robustness of A Feed-Forward Neural Networks in The Presence of Missing Data", IEEE World Congress on Computational Intelligence., IEEE International Conference on 'Neural networks', 2, pp. 836-839, 1994.

Ben-Akiva, M., Cuneo, D., Hasan, M., Jha, M., and Yang, Q., “Evaluation of Freeway Control Using A Microscopic Simulation Laboratory”, Transportation Research Part C: Emerging Technologies 11(1), pp. 29-50, 2003.

Bishop, C. M., Neural Networks for Pattern Recognition, Oxford University Press, United Kingdom, 1995.

Boyce, D., Rouphail, N., and Kirson, A., "Estimation and Measurement of Link Travel Times in the ADVANCE Project", Proceedings of the Vehicle Navigation and Information Systems Conference, IEEE, New York, pp. 62-66, 1993.

Chen, H., Grant-Muller, S., Mussone, L., and Montgomery, F, “A Study Of Hybrid Neural Networks Approaches and The Effects of Missing Data on Traffic Forecasting", Neural Computing and Applications, 10, pp. 277-286, 2001.

Chen, M., and Chien, S. I. J., "Dynamic Freeway Travel-Time Prediction with Probe Vehicle Data - Link Based versus Path Based”, Transportation Research Record 1768, Transportation Research Board, Washington, DC, pp. 157-161, 2001.

Chien, S. I. J. and Kuchipudi, C. M., "Dynamic Travel Time Prediction with Real Time and Historic Data”, Journal of Transportation Engineering, 129(6), pp. 608-616, 2003.

Coifman, B., "Estimating Travel Times and Vehicle Trajectories on Freeways Using Dual Loop Detectors”, Transportation Research. Part A, 36, pp. 351-364, 2002.

Cortes, C. E., Lavanya, R., Oh, J. S., and Jayakrishnan, R., "General-Purpose Methodology for Estimating Link Travel Time with Multiple-Point Detection of Traffic", Transportation Research Record 1802, Transportation Research Board, Washington, DC, pp. 181-189, 2002. 
D’Angelo, M., Al-Deek, H., and Wang, M., "Travel Time Prediction for Freeway Corridors", Transportation Research Record 1676, Transportation Research Board, Washington, DC, pp.184-191, 1999.

Dailey, D. J., "Travel Time Estimates Using A Series of Single-Loop Volume and Occupancy Measurements”, TRB 1997 Annual Meeting CD-ROM, 1997.

Danech-Pajouh, M., and Aron, M., "ATHENA: A Method for Short-term Inter-urban Motorway Traffic Forecasting”, Research Transport Security, 9, pp. 11-16, 1991.

De Vries and Principe, The Gamma Neural Net - A New Model for Temporal Processing. Neural Networks, vol. 5, no. 4, pp. 565-576, 1992.

Dia, H., "An Object-Oriented Neural Networks Approach to Short-Term Traffic Forecasting”, European Journal of Operational Research, 131(2), pp. 253-261, 2001.

Garber, N., and Hoel L., Traffic and Highway Engineering, Third Edition, 2002.

Haj-Salem, H., and Lebacque, J. P., "Reconstruction of False and Missing Data with First-Order Traffic Flow Model”, Transportation Research Record 1802, Transportation Research Board, Washington, DC, pp. 155-165, 2002.

Haykin, S., Neural Networks - A Comprehensive Foundation, Second Edition, PrenticeHall, Inc., 1999.

Hoogendoorn, S. P., "Model-Based Multiclass Travel Time Estimation”, TRB 2000 Annual Meeting CD-ROM, 2000.

Hu, T. Y., "Evaluation Framework for Dynamic Vehicle Routing Strategies under RealTime Information", Transportation Research Record 1774, Transportation Research Board, Washington, DC, pp. 115-122, 2001.

Huisken, G., and Van Berkum, E. C., "A Comparative Analysis of Short-Range Travel Time Prediction Methods”, TRB 2003 Annual Meeting CD-ROM, 2003.

Innamaa, S., "Short-Term Prediction of Travel Time Using Neural Networks on An Interurban Highway”, Transportation, Volume 32, pp, 649-669, 2005.

Ishak, S. and Al-Deek, H., "Performance Evaluation of Short-Term Time-Series Traffic Prediction Model”, Journal of Transportation Engineering, 128(6), pp. 490- 498, 2002.

Ishak, S., Kotha, P., and Alecsandru, C., “Optimization of Dynamic Neural Networks Performance for Short-Term Traffic Prediction”, Transportation Research Record, 1836, Transportation Research Board, Washington, DC, pp. 45-56, 2003. 
Iwasaki, M., and Shirao, K., "A Short Term Prediction of Traffic Fluctuations Using Pseudo Traffic Patterns", $3^{\text {rd }}$ World Congress on Intelligent Transport Systems Conference (CD-ROM), Orlando, Florida, 1996

Kwon, J., Coifman, B., and Bickel, P., “Day-to-Day Travel Time Trends and Travel Time Prediction from Loop Detector Data", Transportation Research Record, 1717, Transportation Research Board, Washington, D.C., pp. 120-129, 2000.

Levin, M., and Tsao, Y. D., "On Forecasting Freeway Occupancies and Volumes", Transportation Research Record, 773, Transportation Research Board, Washington, D.C., pp. 47-49, 1980.

Lindveld, C. D. R., Thijis, R., Bovy, P. H., and Van der Zijpp, N. J., "Evaluation of Online Travel Time Estimators and Predictors", Transportation Research Record, 1719, Transportation Research Board, Washington, D.C., pp. 45-53, 2000

Lingras, P., Sharma, S., and Zhong, M., "Prediction of Recreational Travel Using Genetically Designed Regression and Time-Delay Neural Networks Models", Transportation Research Record, 1805, Transportation Research Board, Washington, DC, pp. 16-24, 2002.

Liu, H., Van Zuylen, H., Van Lint, H., Salomons, M., "Predicting Urban Arterial Travel Time with State-Space Neural Networks and Kalman Filters", Transportation Research Record, 1968, Transportation Research Board, Washington, D.C, pp. 99-108, 2006a.

Liu, Y., Lin, P.-W., Lai, X., Chang, G-L, and Marquess, A., "Developments and Applications of Simulation-Based Online Travel Time Prediction System: Traveling to Ocean City, Maryland”, Transportation Research Record 1959, Transportation Research Board, Washington, D.C, pp. 92-104, 2006b.

Manfredi, S., Salem H. H., and Grol, H. J. M., Development and Application of Coordinated Control of Corridors, Technical Report D9.1, Consortium for the EU Telematics Applications Program TRANSPORT, project TR1017 DACCORD, 1998.

Nanthawichit, C., Nakatsuji, T., Suzuki, H., "Application of Probe-Vehicle Data for RealTime Traffic-State Estimation and Short-Term Travel-Time Prediction on A Freeway", Transportation Research Record 1855, Transportation Research Board, Washington, D.C., pp. 49-59, 2003.

Nam, D. H., and Drew, D. R., "Traffic Dynamics: Methods for Estimating Freeway Travel Times in Real-Time from Flow Measurements", Journal of Transportation Engineering, 122(3), pp. 185-191, 1996.

Nam, D. H., and Drew, D. R., "Analyzing Freeway Traffic under Congestion: Traffic Dynamics Approach”, Journal of Transportation Engineering, 124(3), pp. 208-212, 1998. 
Nam, D. H., and Drew, D. R., “Automatic Measurement of Traffic Variables for Intelligent Transportation Systems Applications”, Transportation Research. Part B, 33, pp. 437-457, 1999.

Oda, T., "An Algorithm for Prediction of Travel Time Using Vehicle Sensor Data", Proceedings of the IEE $3^{\text {rd }}$ International Conference on Road Traffic Control, London, U.K., pp 40-44, 1990.

Palacharla, P. V., and Nelson, P. C., "Application of Fuzzy Logic and Neural Networks for Dynamic Travel Time Estimation”, International Transactions in Operational Research, 6, pp. 145-160, 1999.

Park, D., Messer, C. J., and Urbanik, T. II., "Short-term Traffic Volume Forecasting Using Radial Basis Function Neural Networks”, Transportation Research Record, 1651, Transportation Research Board, Washington, D.C., pp. 39-47, 1998.

Park, D., and Rilett, L. R., "Forecasting Multiple Period Freeway Link Travel Times Using Modular Neural Networks”, Transportation Research Record, 1617, Transportation Research Board, Washington, D.C., pp. 163-170, 1998.

Park, D., Rilett, L. R., and Han, G., “Spectral Basis Neural Networks for Real-Time Link Travel Times Forecasting”, Journal of Transportation Engineering, 125(6), pp. 515-523, 1999.

Park, D., and Rilett, L. R., "Forecasting Freeway Link Travel Times with A Multi-Layer Feed Forward Neural Networks”, Computer Aided Civil and Infrastructure Engineering, 14, pp. 357-367, 1999.

Petty, K. F., Bickel, P., Ostland, M., Rice, J., Schoenberg, F., Jiang, J., and Ritov, Y., "Accurate Estimation of Travel Times from Single-Loop Detectors", Transportation Research. Part A, 32(1), pp. 1-17, 1998.

Rakha, H., and Zhang, W., "Estimating Traffic Stream Space Mean Speed and Reliability from Dual- and Single-Loop Detectors", Transportation Research Record, 1925, Transportation Research Board, Washington, D.C., pp. 38-47, 2005.

Rice, John., and van Zwet, Erik., "A Simple and Effective Method for Predicting Travel Times on Freeways”, IEEE Transactions on Intelligent Transportation Systems, 5(3), pp. 2004.

Rilett, L. R., and Park, D., "Direct Forecasting of Freeway Corridor Travel Times Using Spectral Basis Neural Networks”, Transportation Research Record, 1752, Transportation Research Board, Washington, DC., pp. 140-147, 2001. 
Saito, M., and Watanabe, T., "Prediction and Dissemination System for Travel Time Utilizing Vehicle Detectors", Proceedings of the $2^{\text {nd }}$ World Congress on Intelligent Transportation Systems conference, Yokohama, Japan, 1995.

Schafer, J.L., Analysis of Incomplete Multivariate Data, Chapman \& Hall, London, 1997.

Seki, S., "Travel Time Measurement and Provision System Using AVI Units", Proceedings of the $2^{\text {nd }}$ World Congress on Intelligent Transportation Systems, Yokohama, Japan, 1999.

Singh, A. K. and G. Abu-Lebdeh, "State Space Neural Networks for Travel Time Predictions in Signalized Networks”, TRB 2007 Annual Meeting CD-ROM, 2007.

Smith, B. L., and Demetsky, M. J., "Short-term Traffic Flow Prediction: Neural Networks Approach”, Transportation Research. Record, 1453, Transportation Research Board, Washington, D. C., pp. 98-104, 1994.

Smith, B. L., and Demetsky, M. J., “Traffic Flow Forecasting: Comparison of Modeling Approaches”, Journal of Transportation Engineering, Volume 123, Issue 4, pp. 261-266, 1997.

Smulders, S. A., Messmer, A., and Knibbe, W. J. J., "Real-time Application of METANET in Traffic Management Centers", Proceedingss of the $6^{\text {th }}$ World Congress on Intelligent Transport Systems (ITS), Toronto, Canada, 1999.

Sun, H., Liu, H. X., Xiao, H., He, R. R., Ran, B., “Use of Local Linear Regression Model for Short-term Traffic Forecasting”, Transportation Research Record, 1836, Transportation Research Board, Washington, DC, pp. 143-150, 2007.

Tarko, A., and Rouphail, N. M., "Travel Time Data Fusion in ADVANCE”, Proceedings of the $3^{\text {rd }}$ International Conference on Applications of Advanced Technologies in Transportation Engineering, ASCE, New York, pp. 36-42, 1993.

Thijs, R., Lindveld, C. D. R., Van der Zijpp, N. J., and Bovy, P. H. L., Deliverable D10.3: Final Report of Assessment Results, Volume I , Technical Report D10.3, Consortium for The EU Telematics Applications Program TRANSPORT, Project TR1017 DACCORD, 1998a.

Thijs, R., Lindveld, C. D. R., Van der Zijpp, N. J., and Bovy, P. H. L., Deliverable D10.3: Final Report of Assessment Results, Volume II, Technical Report, Consortium for The EU Telematics Applications Program TRANSPORT, Project TR1017 DACCORD, 1998b. 
Thijs, R., Lindveld, C. D. R., Van der Zijpp, N. J., and Bovy, P. H. L., Evaluation of Travel Time Estimation and Prediction Algorithms: Evaluation Results from The DACCORD Project, Transportation and Planning Section, Delft University of Technology, 1999.

Transport Simulation Systems (TSS). AIMSUN NG User's Manual. Version 5.1.3, Barcelona, Spain, 2006.

Travel Time Data Collection Handbook, Report no. FHWA-PL-98-035, Texas Transportation Institute, College Station, Texas, 1998.

Turner, S. M., “Advanced Techniques for Travel Time Data Collection”, Transportation Research Record 1551, Transportation Research Board, Washington, DC, pp. 51-58, 1996.

Vanajakshi, L. D., Estimation and Prediction of Travel Time From Loop Detector Data for Intelligent Transportation Systems Applications, Ph.D Thesis, Submitted to Civil Engineering for Texas A\&M University, 2004.

Van der Veoort, M., Dougherty, M., and Watson, S., "Combining Kohonen Maps with ARIMA Time-Series Models to Forecast Traffic Flow”, Transportation Research. Part C, 4(5), pp. 307-318, 1996.

Van Lint, J. W. C., Hoogendoorn, S. P., and Van Zuylen, H. J., "Freeway Travel Time Prediction with State-Space Neural Networks - Modeling State-Space Dynamics with Recurrent Neural Networks", Transportation Research Record, 1811, Transportation Research Board, Washington, D.C, pp. 30-39, 2002.

Van Lint, J. W. C., and Van der Zijpp, N. J., "Improving A Travel Time Estimation Algorithm by Using Dual Loop Detectors", Transportation Research Record, 1855, Transportation Research Board, Washington, D.C., pp. 41-48, 2003.

Van Lint, J. W. C., Reliable Travel Time Prediction for Freeways, Ph.D Thesis, Submitted to Civil Engineering for Delft University of Technology, Netherland, 2004.

Van Lint, J. W. C., Hoogendoorn, S. P., and Van Zuylen, H. J., "Accurate Freeway Travel Time Prediction with State-Space Neural Networks under Missing Data”, Transportation Research, Part C: Emerging Technology, 13(5-6), pp.347-369, 2005.

Van Lint, J. W. C., "Reliable Real-Time Framework for Short-Term Freeway Travel Time Prediction”, Journal of Transportation Engineering, 132(12), pp. 921-932, 2006. 
Vythoulkas, P. C., "Alternative Approaches to Short-Term Traffic Forecasting for Use in Driver Information Systems in: Transportation and Traffic Theory”, Proceedings of the $12^{\text {th }}$ International Symposium on Traffic Flow Theory and Transportation, Berkeley, CA, 1993.

Wang,Y., and Nihan, N. L., "Freeway Traffic Speed Estimation with Single-Loop Outputs”, Transportation Research Record 1727, Transportation Research Board, Washington, DC, pp. 120-126, 2000.

Wardop, J. G., "Some Theoretical Aspects of Road Traffic Research”, Proceedingss of the Institute of Civil Engineers, 1-2, pp. 325-378, 1952.

Wu, C-H., C-C. Wei, D-C. Su, M-H. Chang and J-M. Ho, "Travel Time Prediction with Support Vector Regression”, Volume 2, Issue 12-15, Proceedingss of Intelligent Transportation Systems, IEEE, pp. 1438 - 1442, 2003.

Yin, H., Wong, S. C., and Xu, J., "Urban Traffic Flow Prediction Using Fuzzy-Neural Approach”, Tranportation Research. Part C, 10, pp. 85-98, 2002.

Yun, S. Y., Namkoong, S., Rho, J. H., Shin, S. W., and Choi, J. U., “A Performance Evaluation of Neural Networks Models in Traffic Volume Forecasting”, Mathematical and Computer Modeling, 27(9-11), pp. 293-310, 1998.

Zhang, G., Patuwo, E., and Hu, M. Y., "Forecasting with Artificial Neural Networks: The State of The Art”, International Journal of Forecasting, 14, pp. 35-62, 1998.

Zhang, H. M., "Recursive Prediction of Traffic Conditions with Neural Networks", Journal of Transportation Engineering, 126(6), pp. 472-481, 2000.

Zhang, X. Y., and Rice, J. A., "Short Term Travel Time Prediction", Transportation Research. Part C, 11, pp. 187-210, 2003. 
VITA

\section{LUOU SHEN}

Nov. 1978 Born, Hunan Province, P. R. China

\section{EDUCATION}

July $2000 \quad$ B.S. in Civil Engineering

Tongji University, Shanghai, P. R. China

July 2004 M.S. in Civil Engineering

Changsha Science and Technology University, Changsha, P. R. China

August 2008 Doctoral Candidate in Civil Engineering

Florida International University, Miami, FL

EMPLOYMENT

2000 - 2001 Engineer, Shanghai Highway and Bridge Engineering Company, Shanghai, P. R. China

2004 - 2005 Engineer, Guangdong Highway Construction Company, Guangzhou, P. R. China

2000 - 2008 Graduate Research Assistant, Florida International University, Miami, FL

AFFILIATION

ITE, Institute of Transportation Engineers

\section{PUBLICATIONS AND PRESENTATIONS}

1. Luou Shen and Mohammed Hadi. "Estimation of Segment Travel Time Based on Point Traffic Detector Measurements," Submitted to 88th Transportation Research Board Annual Meeting, August 2008.

2. Luou Shen and Mohammed Hadi. "Freeway Travel Time Prediction with Dynamic Neural Networks," Submitted to Transportation Research Part C: Emerging Technologies, June 2008.

3. Mohammed Hadi and Luou Shen. "Use of Operation Data to Evaluate the Benefits and Costs of Advanced Traffic Management Components," Transportation Research Record (In press), 2008. 
4. Chengjun Zhan, Luou Shen, Albert Gan and Mohammed Hadi. "Understanding the Characteristics of Secondary Crashes on Freeways," Presented at 87th Annual Meeting of the Transportation Research Board, Washington, D.C., 2007.

5. Sheng Yang, Luou Shen and Mohammed Hadi. "Risk Analysis to Account for Uncertainty in Benefit-Cost Evaluations of Intelligent Transportation Systems," Transportation Research Record 2035, pp. 187-19, 2007. 